\title{
Infrared laser-absorption sensing for combustion flows
}

\author{
Christopher S. Goldenstein ${ }^{a}$, R. Mitchell Spearrin ${ }^{b}$, Jay. B. Jeffries ${ }^{c}$, and Ronald K. Hanson ${ }^{c}$ \\ ${ }^{a}$ Purdue University, West Lafayette, IN \\ ${ }^{b}$ University of California Los Angeles, Los Angeles, CA \\ ${ }^{c}$ Stanford University, Stanford, CA
}

\begin{abstract}
Infrared laser-absorption spectroscopy (IR-LAS) sensors play an important role in diagnosing and characterizing a wide range of combustion systems. Of all the laser-diagnostic techniques, LAS is arguably the most versatile and quantitative, as it has been used extensively to provide quantitative, species-specific measurements of gas temperature, pressure, composition and velocity in both laboratory- and industrial-scale systems. Historically, most IR-LAS work has been conducted using tunable diode lasers, however, today's researchers have access to a wide range of light sources that provide unique sensing capabilities and convenient access to nearly the entire IR spectrum $(\approx 1$ to $20 \mu \mathrm{m})$. In particular, the advent of room-temperature wavelength-tunable mid-infrared semiconductor lasers (e.g., interband- and quantum-cascade lasers) and hyperspectral light sources (e.g., Fourierdomain mode-locked lasers, dispersed supercontinuum lasers, and frequency combs) has provided a number of unique capabilities that combustion researchers have exploited. The primary goal of this review paper is to document the recent development, application, and current capabilities of IR-LAS sensors for laboratory- and industrial-scale combustors and propulsion systems. A thorough review and description of the fundamental spectroscopy governing the accuracy of such sensors, and recent findings and databases that enable improved modeling of molecular absorption spectra will be provided. Modern light sources and the most commonly used diagnostic techniques are also discussed.
\end{abstract}

Keywords: laser absorption spectroscopy, molecular spectroscopy, combustion, propulsion

Preprint submitted to Progress in Energy and Combustion Science

July 24, 2016

(C) 2016. This manuscript version is made available under the Elsevier user license

http://www.elsevier.com/open-access/userlicense/1.0/ 


\section{Contents}

1 Introduction $\quad 4$

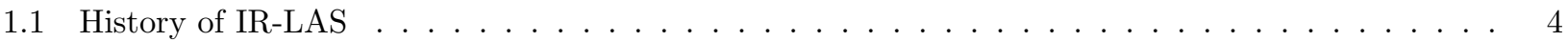

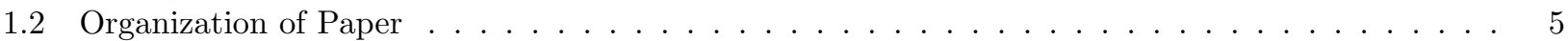

$5 \quad 2$ Fundamentals $\quad 5$

3 Spectroscopic Models and Databases $\quad 7$

3.1 Lineshape Physics and Models . . . . . . . . . . . . . . . . . . . . . 7

3.1.1 Doppler Broadening and the Gaussian Profile . . . . . . . . . . . . . . . . . 7

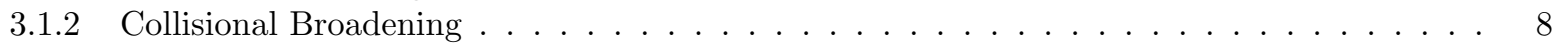

3.1.3 Combined Doppler- and Collisional-Broadening and the Voigt Profile . . . . . . . . . 8

3.1.4 Dicke Narrowing and the Rautian and Galatry Profiles . . . . . . . . . . . . . . . . . 9

3.1.5 Speed-Dependent Collisional Broadening and the Speed-Dependent Voigt Profile . . . . 10

3.1.6 The Impact Approximation and the $\chi$-Function $\ldots \ldots \ldots$. . . . . . . . . . . . 10

3.1 .7 Line Mixing . . . . . . . . . . . . . . . . . . . . . . . . . . . . . . 10

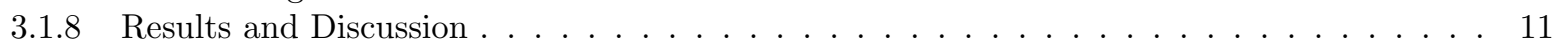

3.2 Databases . . . . . . . . . . . . . . . . . . . . . . . . . . 12

3.3 Line-by-line data . . . . . . . . . . . . . . . . . . . . . . . . . . . . . 12

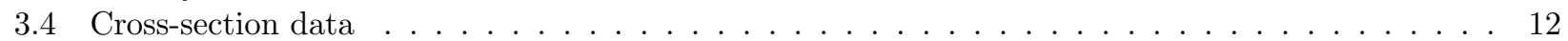

3.5 Uncatalogued data . . . . . . . . . . . . . . . . . . . . . . . . . . 13

4 Techniques 13

4.1 Scanned-Wavelength Direct-Absorption Spectroscopy . . . . . . . . . . . . . . . . . . . . 14

4.2 Hyperspectral Direct-Absorption Spectroscopy . . . . . . . . . . . . . . . . . . . . . . 14

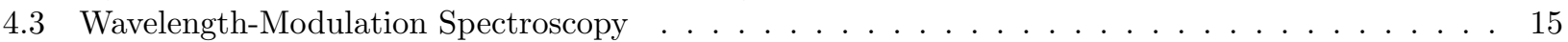

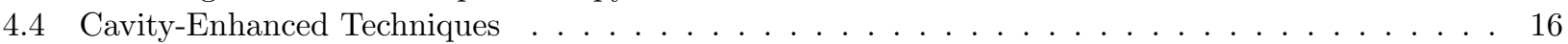

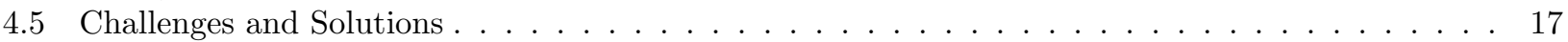

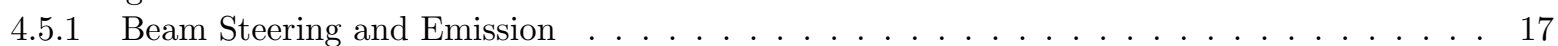

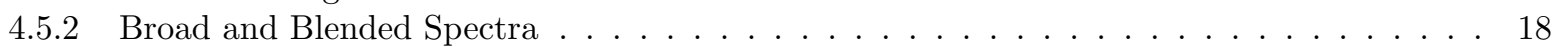

4.5.3 Breakdown of the Impact Approximation . . . . . . . . . . . . . . . . . . . . . . 19

4.5.4 Non-Zero WMS Background . . . . . . . . . . . . . . . . . . . . . . . . 19

4.5.5 $1 f$-Normalization with Large Absorbance . . . . . . . . . . . . . . . . . . 19

5 Solutions for Nonuniform Flows $r$

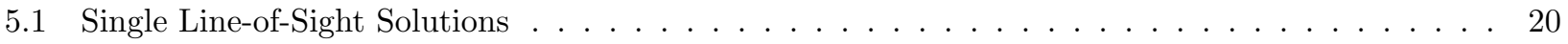

5.2 Laser-Absorption Tomography . . . . . . . . . . . . . . . . . . . . . . . . 20

6 Sources and Hardware $\quad 21$

6.1 Near-Infrared Hardware . . . . . . . . . . . . . . . . . . . . . . . . . . . 21

6.1.1 Distributed-Feedback (DFB) Tunable Diode Lasers (TDLs) . . . . . . . . . . . . . . . 21

6.1 .2 Vertical Cavity Surface Emitting Lasers (VCSELs) . . . . . . . . . . . . . . . . . . 22

6.1 .3 Fourier-Domain Mode Locked (FDML) Lasers . . . . . . . . . . . . . . . . . . . . 22

6.1 .4 Dispersed Supercontinuum Lasers _. . . . . . . . . . . . . . . . . . . 22

6.1 .5 Near-Infrared Optical Fibers . . . . . . . . . . . . . . . . . . . . . . 23

6.2 Mid-Infrared Hardware . . . . . . . . . . . . . . . . . . . . . . . . . . . . . 23

6.2 .1 Quantum Cascade Lasers (QCLs) . . . . . . . . . . . . . . . . . . . 23

6.2 .2 Interband Cascade Lasers (ICLs) . . . . . . . . . . . . . . . . . . . . . . . 24

6.2 .3 Mid-IR Optical Fibers . . . . . . . . . . . . . . . . . . . . . . . . . . . . . 24

6.2 .4 Mid-IR Optical Materials . . . . . . . . . . . . . . . . . . . . . . . . 24

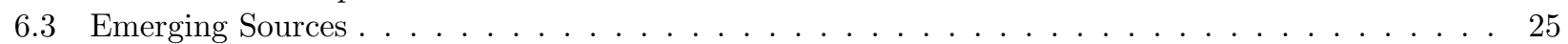

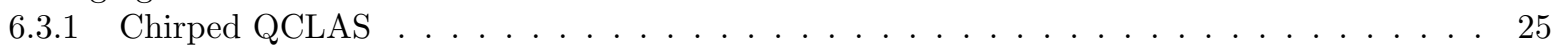

6.3 .2 Frequency Comb Spectrometers . . . . . . . . . . . . . . . . . . . 26

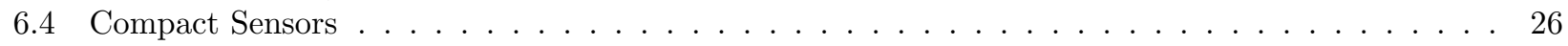


6.4 .2 Single-Ended Sensors Using Native Surfaces . . . . . . . . . . . . . . . . . . 28

7 Sensors for Fundamental Combustion Chemistry Studies 28

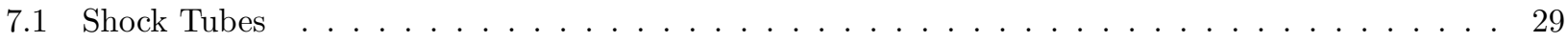

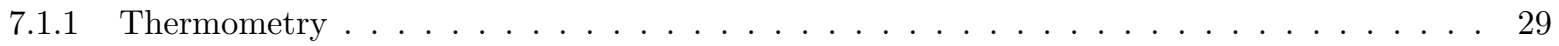

7.1 .2 High-Pressure Studies and Sensor Development . . . . . . . . . . . . . . . . . 29

7.1 .3 Fuel Intermediates $\ldots \ldots \ldots \ldots$

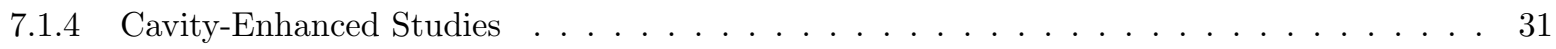

7.2 Laboratory Flames . . . . . . . . . . . . . . . . . . . . . . . . 31

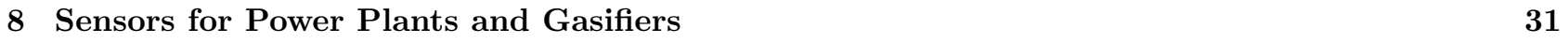

609 Sensors for Gas Turbines $\quad 33$

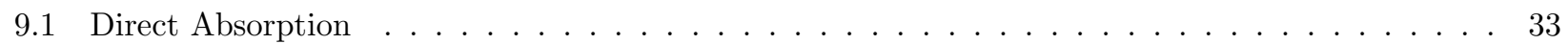

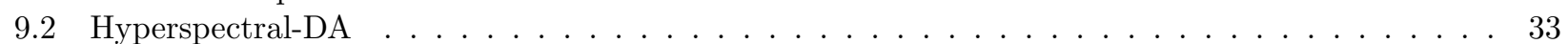

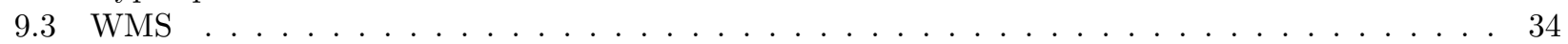

10 Sensors for IC Engines $\quad 35$

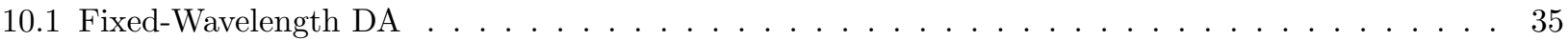

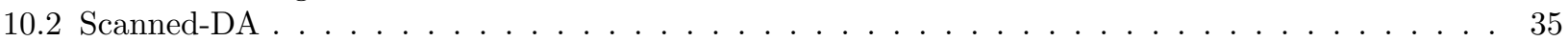

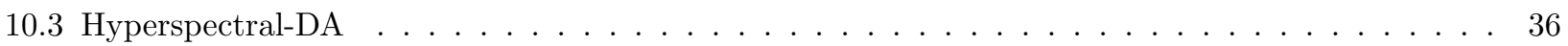

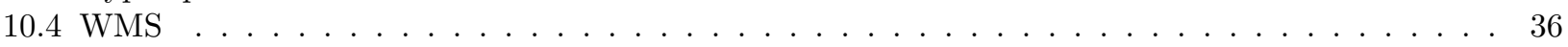

11 Sensors for Scramjets $\quad 37$

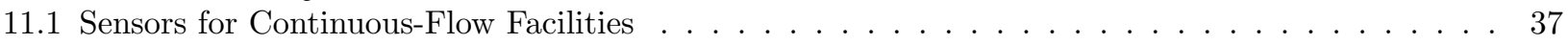

11.2 Sensors for Impulse Facilities $\ldots \ldots \ldots \ldots$

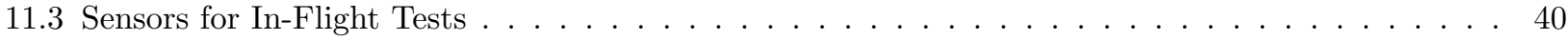

12 Sensors for Pulsed-Detonation Combustors $\quad 40$

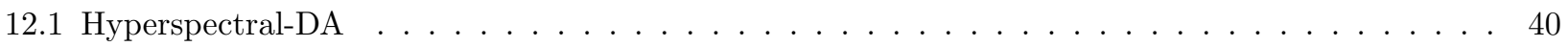

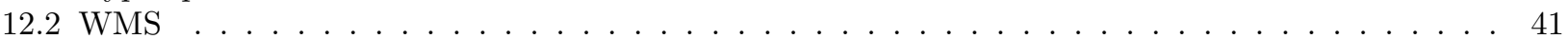

13 Sensors for Rotating-Detonation Combustors 43

14 Conclusions and Outlook $\quad 45$ 


\section{Introduction}

\subsection{History of IR-LAS}

80 Over the last $40+$ years, a wide range of laser diagnostics have been developed and deployed for characterizing combustion via measurements of gas temperature, pressure, velocity, and composition. The development and application of these measurement methods

85 has been highlighted in diagnostics colloquia during the past twenty bienniel International Combustion Symposia (published in the Proceedings of the Combustion Institute), as well as in several excellent review articles and text books. For example, applications of laser 90 diagnostics have been an important part of two Hottel papers at the International Combustion Symposium (1998 by Wolfrum [2] and 2010 by Hanson [3]) as well as numerous other reviews such as those by Allen [4], Werle [5], Lackner [6], and Schulz et al. [7], and most 95 recently, Bolshov et al. [8]. Several books have als8 been devoted to this subject. For example, Eckbreth [9] describes the early history and the basic fundamental science of laser-diagnostic techniques, Kohse and Jeffries [10] provide a review of practical applications, and most recently, Hanson, Spearrin, and Goldenstely [11] provide a thorough description of the fundamental spectroscopy and physics needed by researchers and students to apply laser-based diagnostics to their work. This review will focus on documenting the status of in-

105 frared laser-absorption spectroscopy (IR-LAS) sensoriso, and the role they play in studying combustion science and characterizing combustion-based systems.

While the spatial resolution of IR-LAS is typically limited by its path-integrated nature, it is arguably diagnostic technique. Its comparatively simple signal interpretation enables quantitative measurements of gas temperature, pressure, velocity and nearly all molecular species (excluding homonuclear diatomics, 15 although a low-lying electronic state makes $\mathrm{O}_{2}$ an exception). Furthermore, IR-LAS can be applied to virtually all temperatures and pressures without any fundamental limitation (e.g., collisional quenching). Perhaps most importantly, lasers and optical hardware stricted access throughout the IR ( 1 to $20 \mu \mathrm{m})$, with much of this hardware enabling the development of robust sensor packages suitable for field testing. However, like all methods, IR-LAS is not free from com125 plications and a wide-range of measurement challenges complicate its development and use. This review will highlight these challenges and the exceptional work done by many researchers to overcome them. First we will review some of the early milestones that have shaped the development of IR-LAS.

Soon after the laser was invented, Sulzmann [12] recognized the value of laser-absorption measurements of specific species in combustion gases, and the first applications using tunable diode lasers (TDLs) soon followed. In the late 1970s, TDLs and infrared detectors required cryogenic cooling and dispersive optics were required to select a single mode from the multimode laser output. To our knowledge the first application of TDLs for spectrally resolved direct-absorption sensing in flames was reported by Hanson in 1977 [13]. These methods were quickly extended to shockheated gases [14] and two-color thermometry in flame gases [15]. Not long after, frequency- and wavelengthmodulation spectroscopy (FMS and WMS) were developed to increase the sensitivity of TDL absorption $[16,17,18,19,20,21,22,23]$.

Ironically, the first IR-LAS measurements were conducted in the mid-infrared (mid-IR), although the associated hardware limitations at the time severely complicated or precluded measurements outside of the laboratory. This began to change in the mid 1980s, when the telecommunication industry began producing large numbers of AlGaAs and InGaAsP diode lasers that could be operated at room temperature. In 1988, Cassidy leveraged these advancements and used a fibercoupled room-temperature TDL near $1.3 \mu \mathrm{m}$ for tracegas sensing via WMS [24]. In 1991, room temperature TDLs were first applied to gasdynamic applications via the the development of the first diode-laser-based mass flux sensor based on spectrally resolved $\mathrm{O}_{2}$ absorption [25], a technique which would be used in-flight 20 years later [26, 27, 28]. In 1993 Arroyo and Hanson [29] became the first to use telecommunication TDLs near $1.4 \mu \mathrm{m}$ for two-color thermometry and $\mathrm{H}_{2} \mathrm{O}$ sensing in flames. Subsequent advancements leveraging telecommunication technology led to wavelength-multiplexed and fiber-coupled sensors for multi-parameter sensing $[30,31]$, and the first TDLAS sensor for closed-loop combustor control [32]. Even though the TDL industry was in its infancy, there were many early applications to combusting flows and the reader is referred to several excellent reviews by Allen [4], Werle [5], and Wolfrum [2].

Research in the 2000s focused on improving the robustness of LAS sensors for field measurements via increased spectral information and use of improved WMS techniques, and these trends continue today. The enhanced wavelength tuning capabilities of VCSELs were exploited for the first high-pressure measurements of $\mathrm{O}_{2}$ [33], as well as cesium spectra for temperature, pressure, and velocity measurements in detonation flows at high rates $[34,35]$. Soon after Fourier-domain mode-locked (FDML) lasers were used to acquired even greater spectral information of $\mathrm{H}_{2} \mathrm{O}$ near $1.4 \mu \mathrm{m}$ for thermometry and $\mathrm{H}_{2} \mathrm{O}$ sensing in a variety of combustion flows $[36,37,38]$. Around the same time, TDLbased sensors for combustion and propulsion applica- 

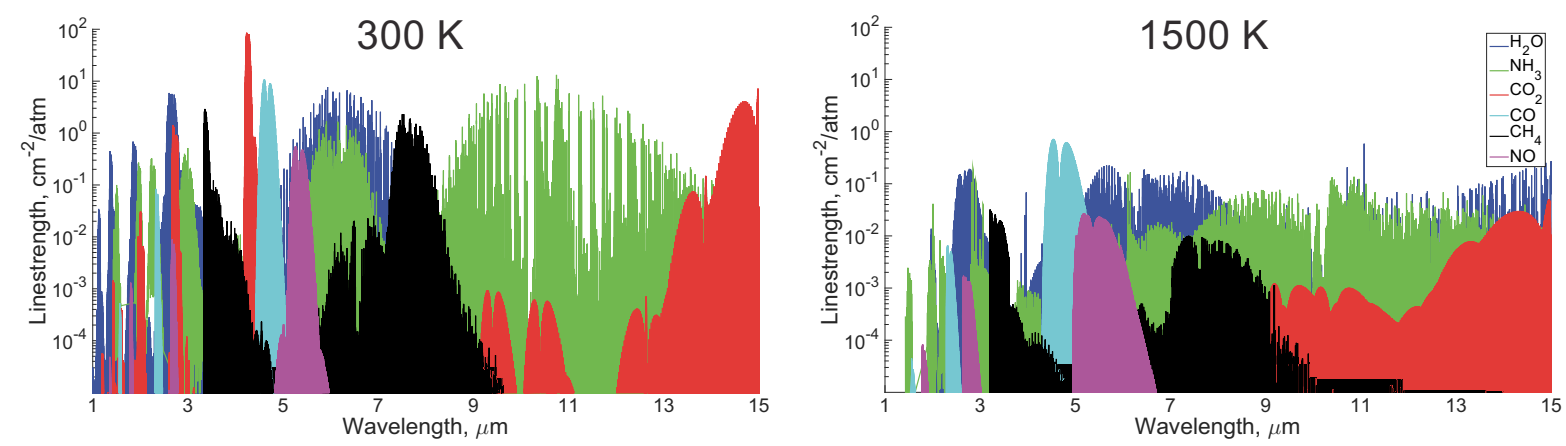

Figure 1: Linestrengths (i.e., absorption strength) of several molecular species of interest to combustion systems. Calculations performed using the HITRAN2012 database [1] with some erroneous linestrengths removed.

tions shifted towards WMS owing to the development of improved, calibration-free WMS models [39, 40, 41] order intensity modulation accompanying it, as well as $1 f$-normalization. These methods were first applied tв TDLAS sensors for temperature and $\mathrm{H}_{2} \mathrm{O}$ sensing in an IC-engine [42] and a model-scramjet combustor [43].

Today researchers developing IR-LAS techniques and sensors have never had more design freedom. Nearly any wavelength in the IR can be accessed bys single-mode, room-temperature lasers enabling sensitive monitoring of most molecular species of interest to combustion. Figure 1 shows the intensity of various IR absorption transitions for a few combustion species at 300 and $1500 \mathrm{~K}$. Unrestricted access to the IR spectrum has provided unprecedented opportunities for the development of LAS sensors that are tailored for unique tions to optimize signal strength and temperature sensitivity, and minimize interfering absorption. These compact, solid-state laser sources include diode lasers (TDLs, visible to $\approx 3 \mu \mathrm{m}$ ), interband cascade lasers (ICLs, 3 to $5 \mu \mathrm{m}$ ), and quantum cascade lasers (QCLs, ture or thermoelectric-cooled detectors. In addition, wide variety of hyperspectral lasers continue to be developed, including FDML, dispersed supercontinuum, 215 frequency combs, and external-cavity TDLs and QCLs. With these tools, there are few molecules that cannot be measured, which has taken us to an exciting time focused not only on the fundamental development of new LAS techniques, but on the transitioning of new LAS sensors into the field.

\subsection{Organization of Paper}

The goals of this review are: 1) to thoroughly explain the underlying fundamentals of IR absorption spectroscopy and the most widely used LAS tech- ing the application of IR-LAS techniques and sensors to combustion-related studies, and 3) to highlight the emerging sensor technologies and the needs and opportunities facing IR-LAS researchers today. We begin this paper by reviewing the basic fundamentals of LAS and follow with a comprehensive discussion of the various absorption-spectroscopy models and databases available to researchers today. Next, we discuss three of the most widely used IR-LAS techniques: direct absorption (DA), wavelength modulation spectroscopy (WMS), and cavity-enhanced absorption spectroscopy (CEAS), where we consider fixed- and scanned-wavelength variants of these methods. We then discuss the optical hardware (i.e., lasers, fibers, IR materials etc.) available today, and the role they play in various IR-LAS techniques. We then review and highlight some of the most impressive examples of IR-LAS applied to combustion and combustionbased systems; sensors for: flames, shock tubes, power plants, gasifiers, gas turbines, internal combustion engines, scramjets, and detonation combustors will all be discussed. Our review is primarily limited to work done in the last 15 years (to avoid repeating the excellent, earlier reviews), with particular emphasis on the last 5 years. Lastly, we conclude with a brief discussion on the outlook, needs, and opportunities for IR-LAS in combustion research.

\section{Fundamentals}

The fundamentals of LAS are described to establish the conventions and units used throughout this manuscript and facilitate the discussion of more complex theory and diagnostic techniques that follow.

Atoms and molecules with a dipole moment can absorb and emit radiation. In the infrared, absorption and emission can occur when the photon energy is equal (i.e., resonant) to the spacing between two discrete ro-vibrational states and the amount of absorbed 
or emitted radiation is proportional to the number density in the absorbing or emitting states, respectransitions within a given vibrational band (a group of transitions corresponding to a specific change in vibrational energy) and each individual transition within that band corresponds to a unique change in rotaare broadened by several mechanisms and a lineshape model is required to describe the distribution (in energy/wavelength/frequency space) over which a given transition absorbs or emits light. These concepts are 275 illustrated in Figure 2.

For a monochromatic light source, the light intensity transmitted through a uniform, absorbing gas is given by Beer's Law in the following form:

$$
I_{t}=I_{o} \exp \left(-k_{\nu} L\right)
$$

where $I_{o}$ and $I_{t}$ are the incident and transmitted light cient per unit length at optical frequency $\nu$, and $L[\mathrm{~cm}]$ is the path length through the absorbing gas. This formulation is valid for all cases where $k_{\nu}$ is constant along the measurement path (e.g., uniform gas properties and negligible absorption-induced population transfer). In this case, the absorbance $\left(\alpha=k_{\nu} L\right)$ can be related gas properties using

$$
\alpha(\nu)=\sum_{j} S_{j}(T) P \chi_{A b s} \phi_{j}(\nu, T, P, \chi) L
$$

where $S(T)\left[\mathrm{cm}^{-2}\right.$-atm $\left.{ }^{-1}\right]$ is the linestrength as a function of temperature $T[\mathrm{~K}], P$ [atm] is the gas pressure, $\phi$ $[\mathrm{cm}]$ is the lineshape function, and $j$ denotes a specific absorption transition. In combustion gases, the lineshape function is usually modeled by the Voigt Profile (VP) owing to it accounting for both Doppler and collisional broadening. However, in certain cases (discussed 295 in Section 3) the VP can lead to substantial errors aned more advanced lineshape models are more appropriate.

Typically $\phi$ is defined according to Eq. 3 such that the integrated absorbance, $A$, is given by Eq. 4 . When individual transitions can be resolved, the integrated gas properties, thereby avoiding the need for a priori knowledge of line broadening.

$$
\begin{gathered}
\int_{-\infty}^{+\infty} \phi d \nu=1 \\
A_{j}=S_{j}(T) P \chi_{A b s} L
\end{gathered}
$$

For a gas in thermal equilibrium, the transition linestrength, $S(T)$, is given by:

$$
\begin{gathered}
S(T)=S\left(T_{o}\right) \frac{Q\left(T_{o}\right)}{Q(T)} \frac{T_{o}}{T} \exp \left[-\frac{h c E^{\prime \prime}}{k}\left(\frac{1}{T}-\frac{1}{T_{o}}\right)\right] \\
{\left[1-\exp \left(-\frac{h c \nu_{o}}{k T}\right)\right]\left[1-\exp \left(-\frac{h c \nu_{o}}{k T_{o}}\right)\right]^{-1}}
\end{gathered}
$$

where $T_{o}$ is the reference temperature $(296 \mathrm{~K}), Q$ is the partition function of the absorbing species, $h[\mathrm{~J}-\mathrm{s}]$ is Planck's constant, $k\left[\mathrm{~J}_{-} \mathrm{K}^{-1}\right]$ is the Boltzmann constant, $c\left[\mathrm{~cm} \mathrm{~s}^{-1}\right]$ is the speed of light, $E^{\prime \prime}\left[\mathrm{cm}^{-1}\right]$ is the lower-state energy of the transition, and $\nu_{o}\left[\mathrm{~cm}^{-1}\right]$ is the transition linecenter frequency.

The measured integrated absorbance of a transition can be used to calculate the mole fraction of the absorbing species (with temperature, pressure, and path length known) and the gas temperature can be calculated directly from a measured two-color ratio of integrated areas using Eq. 6 [11].

$$
T=\frac{\frac{h c}{k}\left(E_{2}^{\prime \prime}-E_{1}^{\prime \prime}\right)}{\ln R+\ln \frac{S_{2}\left(T_{o}\right)}{S_{1}\left(T_{o}\right)}+\frac{h c}{k} \frac{E_{2}^{\prime \prime}-E_{1}^{\prime \prime}}{T_{o}}}
$$

Here $R$ is the two-color ratio of integrated areas for transitions 1 and 2. Note that Eq. 6 depends only on $\mathrm{R}$ and spectroscopic parameters and is independent of pressure, mole fraction, and path length.

In addition, the velocity of a gaseous flow along a line-of-sight can be determined by measuring the Doppler-shifted absorption. In practice, this is typically done using two laser beams, e.g., angled at $45^{\circ}$ and $135^{\circ}$ relative to the flow (i.e., one directed upstream and one downstream), to enable use of the differential Doppler-shift. In this case, the flow velocity, $U$, can be calculated using Eq. 7:

$$
U=\frac{\Delta \nu}{\nu_{o}} \frac{c}{2 \sin \left(45^{\circ}\right)}
$$

where $\Delta \nu$ is the difference between the Doppler-shifted transition linecenter measured across each line-of-sight.

For molecules with unresolved rotational spectra (e.g., large hydrocarbons such as heptane) it is more convenient to use Eq. 8 to relate the absorbance to gas properties.

$$
\alpha(\nu)=n \chi_{A b s} \sigma_{A b s}(\nu, T, P, \chi) L
$$

Here $n\left[\right.$ molecules- $\left.\mathrm{cm}^{-3}\right]$ is the number density of the gas and $\sigma\left[\mathrm{cm}^{2}\right.$-molecule $\left.{ }^{-1}\right]$ is the absorption cross section of the absorbing species. The key difference between Eq. 2 and 8 is that Eq. 2 calculates the spectral absorbance by modeling the contribution of each individual absorption transition whereas Eq. 8 avoids this complexity via use of an absorption cross section which quantifies how much light a molecule absorbs at a given wavelength and thermodynamic conditions. While the 


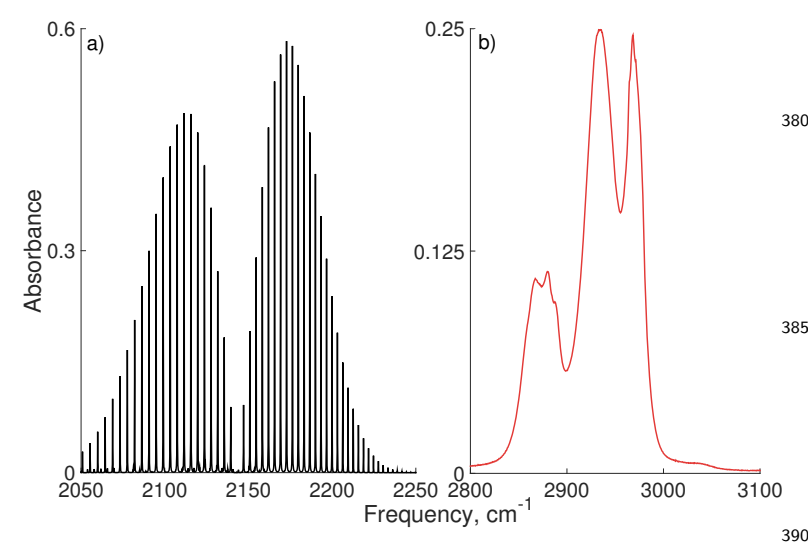

Figure 2: $\quad$ Simulated absorbance spectra of $\mathrm{CO}$ near $4.7 \mu \mathrm{m}$ [1] (a) and heptane near $3.4 \mu \mathrm{m}$ [44] (b) at 1 atm, $298 \mathrm{~K}, 1 \%$ absorber by mole in air, across a $1 \mathrm{~cm}$ path.

latter approach is less complex, it relies on a lookup not be extrapolated beyond the thermodynamic conditions for which the cross sections were obtained. In comparison, the former approach can be used to calculate the absorbance at an arbitrary thermodynamic condition as long as the fundamental spectroscopic parameters for the active transitions (e.g., linestrength, collisional-broadening coefficients) are available. Fig. 2 shows simulated absorbance spectra of the fundamental vibration band of $\mathrm{CO}$ (a) and the $\mathrm{C}-\mathrm{H}$ stretch

355 of heptane (b). The discrete absorbance spectrum of CO's fundamental vibration band was calculated using Eq. 2 and line-by-line parameters [1]. The absorbance spectrum of heptane's C-H stretch absorption band was calculated using Eq. 8 and the absorption cross section measured by Klingbeil et al. [44].

\section{Spectroscopic Models and Databases}

Line-by-line simulations of absorption spectra require 1) a spectroscopic model and 2) spectroscopic parameters that describe how the line positions, section will focus on discussing lineshape-physics and -models since the determination of linestrengths is relatively straightforward and most LAS techniques do not require precise knowledge of absolute line positions.

370 Our discussion will focus on models that are applicable to cases where the assumption of isolated lines (i.e., no line mixing) and the impact approximation are valid, however, both of these complexities will be touched on. This section will then conclude with a discussion on the status and availability of various spectroscopic databases with particular attention to those that an most relevant to elevated temperatures and pressures.

\subsection{Lineshape Physics and Models}

In the vast majority of combustion applications, researchers have used the Voigt profile to model absorption lineshapes owing to its relative simplicity and, typically, high accuracy, yielding residuals within $2 \%$ of measured lineshapes at number densities near STP. However, many researchers have shown that the Voigt profile can lead to gull-wing shaped residuals (between measured and model spectra, shown later in Fig. 4a) on the order of $1-10 \%$ of the peak absorbance at low to moderate number densities (i.e., when collisional narrowing or speed-dependent broadening can be significant). Furthermore, it is also well known that the Voigt and Lorentzian profiles breakdown at high number densities due line mixing and a breakdown of the impact approximation.

This section will discuss the underlying collision physics responsible for these complexities, the lineshape models that address them, and will point the reader to the relevant literature. Particular attention will be paid to $\mathrm{H}_{2} \mathrm{O}$ and $\mathrm{CO}_{2}$, however, more generally these molecules can be thought of as ambassadors for other molecules with large or small rotational-energylevel spacing, respectively. Table 1 introduces a few of these processes and points out the somewhat fortuitous nature of how molecules with large rotationalenergy level spacing are prone to only Dicke narrowing and speed-dependent broadening complications, whereas molecules with small rotational-energy-level spacing are prone to only line mixing and the breakdown of the impact approximation. Table 2 illustrates the parameters needed by each lineshape model discussed in this section.

Table 1: Importance of various collisional processes influencing IR spectra of $\mathrm{H}_{2} \mathrm{O}$ and $\mathrm{CO}_{2}$.

\begin{tabular}{lll}
\hline Process & $\mathrm{H}_{2} \mathrm{O}$ & $\mathrm{CO}_{2}$ \\
\hline Dicke Narrowing & $\mathrm{High}$ & Low \\
Speed-Dependent Broadening & High & Low \\
Impact Approximation & Low & High \\
Line Mixing & Low & High \\
\hline
\end{tabular}

\subsubsection{Doppler Broadening and the Gaussian Profile}

Doppler broadening occurs when molecules in the absorbing state have a velocity component in the direction of the photon propagation. The absorbing molecules then see a Doppler-shifted frequency and will absorb radiation when the Doppler-shifted frequency is resonant with the absorption transition. Since the absorbers are distributed across a range of velocities (i.e., they may belong to different velocity classes), Doppler broadening is said to be heterogeneous. In the absence of other broadening mechanisms, the absorp- 
tion lineshape of gases following a Maxwellian velocitydistribution function (VDF) is given by the Gaussian profile, $\phi_{D}$ :

$$
\phi_{D}(\nu)=\frac{2}{\Delta \nu_{D}}\left(\frac{\ln 2}{\pi}\right)^{1 / 2} \exp \left[-4 \ln 2\left(\frac{\nu-\nu_{o}}{\Delta \nu_{D}}\right)^{2}\right]
$$

where the Doppler full-width at half-maximum (FWHM), $\Delta \nu_{D}$, is given by Eq. 10 .

$$
\Delta \nu_{D}=7.1623 \times 10^{-7} \nu_{o} \sqrt{T / M}
$$

Here, $M$ is the molecular weight of the absorbing species in $\mathrm{g} / \mathrm{mol}$ and $T$ is in K. Eq. 10 indicates that the Doppler FWHM scales linearly with the transition 430 linecenter frequency and with $\sqrt{T / M}$ indicating that Doppler broadening is largest for high-frequency transitions, light-weight absorbers, and high temperatures

\subsubsection{Collisional Broadening}

Collisional broadening of an IR absorption transition when: 1) inelastic collisions reduce a molecule's lifetime in the absorbing state, 2) elastic dephasing collisions perturb the molecular rotation or vibration, or 3) elastic angular-momentum altering collisions reorient the angular momentum vector of the dipole [45].

440 Collisional broadening is typically modeled as homogeneous, meaning the broadening process is independent of the absorber's speed. In this case, collisional broadening is accurately described by a Lorentzian profile, $\phi_{L}$, and an ensemble-averaged collisional FWHM, $\Delta \nu_{c}$, given by Eq. 11 and 12, respectively.

$$
\begin{gathered}
\phi_{L}(\nu)=\frac{1}{2 \pi} \frac{\Delta \nu_{C}}{\left(\nu-\nu_{o}\right)^{2}+\left(\frac{\Delta \nu_{C}}{2}\right)^{2}} \\
\Delta \nu_{c}=2 P \sum_{k} \chi_{k} \gamma_{k}(T)
\end{gathered}
$$

Here $\gamma_{k}$ is the temperature-dependent ensembleaveraged collisional-broadening coefficient of perturbing-species $k$.

The temperature-dependence of $\gamma_{k}$ is usually modeled via a power-law, Eq. 13.

$$
\gamma_{k}(T)=\gamma_{k}\left(T_{o}\right)\left(T_{o} / T\right)^{n_{\gamma}}
$$

Here $n_{\gamma}$ is the collisional-broadening temperature es ponent. It should be noted that the accuracy of Eq. 13 can breakdown over a large temperature range, particularly when the parameters are used beyond the tem455 perature domain for which they were obtained. In our experience, usually a single value of $n_{\gamma}$ is sufficient f®a temperatures from 300 to $1200 \mathrm{~K}$, however, for greater accuracy we recommend using multiple values of $n_{\gamma}$, one for temperatures from 300 to $900 \mathrm{~K}$ and another

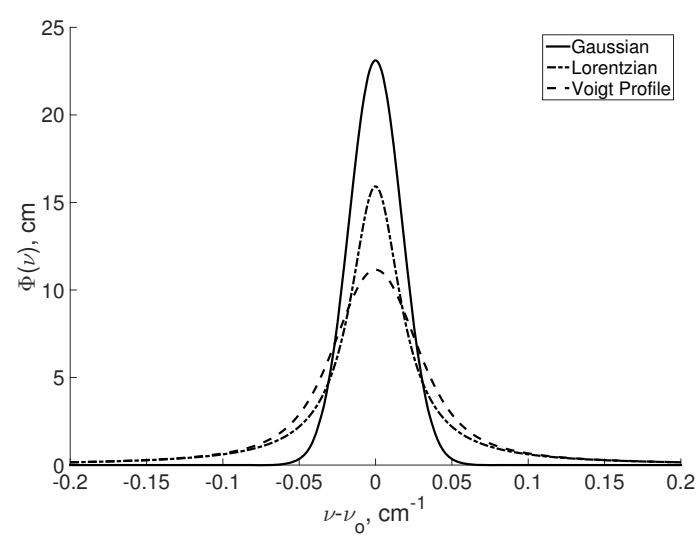

Figure 3: Simulated Gaussian, Lorentzian, and Voigt profiles with $\Delta \nu_{D}=\Delta \nu_{c}=0.04 \mathrm{~cm}^{-1}$.

for temperatures greater than $900 \mathrm{~K}$, however, these are not steadfast rules.

\subsubsection{Combined Doppler- and Collisional-Broadening and the Voigt Profile}

The Voigt profile (VP) is given by a convolution of the Gaussian and Lorentzian profiles and, therefore, accounts for both Doppler and collisional broadening. To date, the Voigt profile remains the lineshape of choice for most spectroscopic databases (e.g., HITRAN [1] and HITEMP [47]) owing to its wide applicability and balance between simplicity and accuracy. In practice, the Voigt profile is typically calculated using numerical approximations due to the computational cost of evaluating it directly. For example, algorithms developed by Humlicek [48] (later enhanced by Kuntz [49] and corrected by Ruyten [50]) and that of McClean et al. [51] are some of the most widely used approximations. The Voigt profile reduces to the Gaussian and Lorentzian profiles when $\Delta \nu_{c}$ or $\Delta \nu_{D}$ equal 0 , respectively. However, numerical approximations can breakdown in these limits, therefore the user should be cautious.

To illustrate the lineshape profiles discussed thus far, Figure 3 shows Gaussian, Lorentzian, and Voigt Profiles with $\Delta \nu_{D}=\Delta \nu_{c}=0.04 \mathrm{~cm}^{-1}$ (i.e., representative values of near-IR $\mathrm{H}_{2} \mathrm{O}$ transitions dilute in air at 1 atm and $1000 \mathrm{~K}$ ). Despite having equal FWHM, the Gaussian profile exhibits a considerably larger peak absorbance compared to the Lorentzian profile, thereby highlighting the non-equal nature of these broadening mechanisms. Furthermore, the FHWM of the Voigt profile is $0.066 \mathrm{~cm}^{-1}$ (i.e., representative of the convolution of Gaussian and Lorentzian distributions and, thus, is not equal to the sum of Doppler and collisional FWHM). 
a)

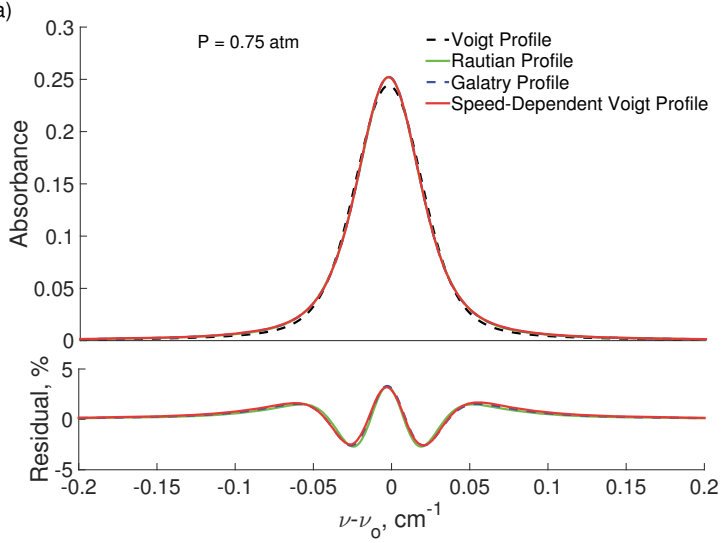

b)

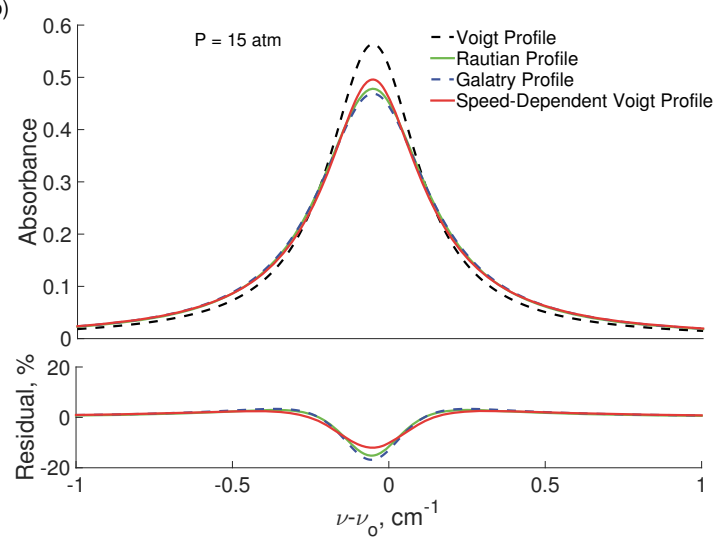

Figure 4: Best-fit lineshapes for $\mathrm{H}_{2} \mathrm{O}$ transition near $7472.21 \mathrm{~cm}^{-1}\left(J^{\prime \prime}=16\right)$ in $\mathrm{N}_{2}$ at $1100 \mathrm{~K}, 0.75$ atm (a) and simulated lineshapes (using best-fit parameters) at $1100 \mathrm{~K}$ and $15 \mathrm{~atm}$ (b). Lineshape parameters were taken from [46]. The residual is between the Voigt profile and all other lineshapes (quoted as percent of peak absorbance). Figures adapted from [46].
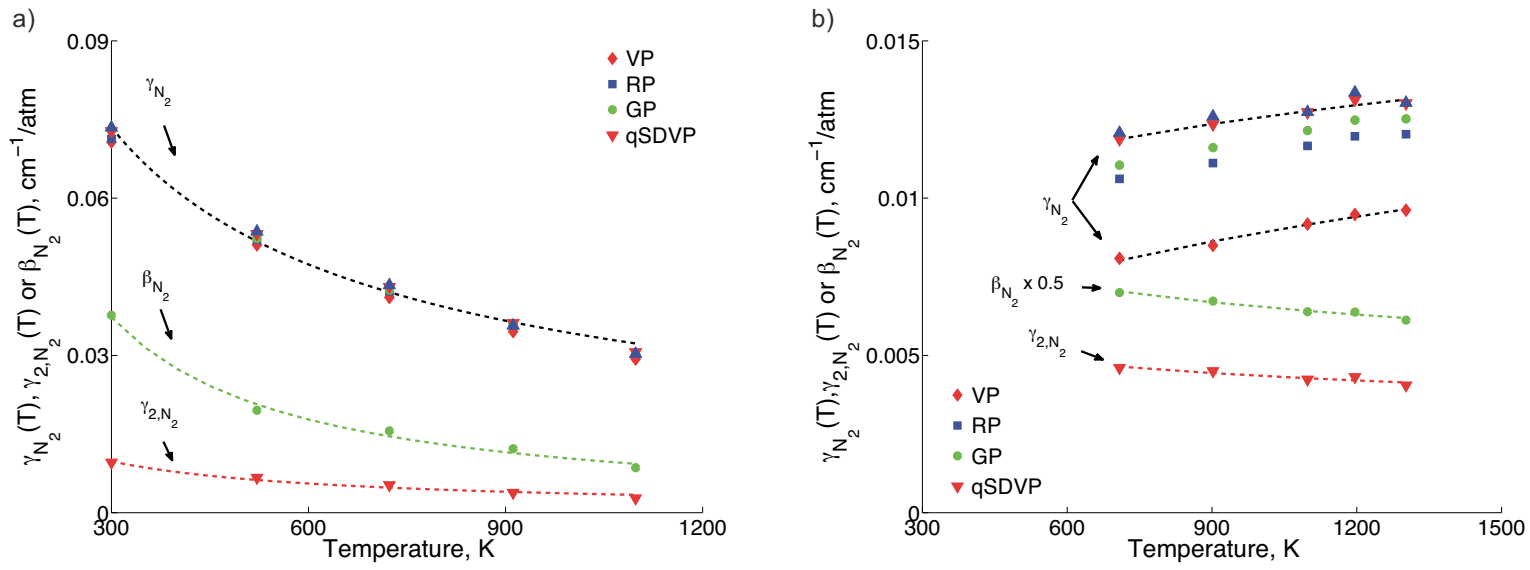

Figure 5: Best-fit lineshape parameters as a function of temperature for an $\mathrm{H}_{2} \mathrm{O}$ transition near $7413.02 \mathrm{~cm}^{-1}$ with $J^{\prime \prime}=8$ (a) and near $7471.7 \mathrm{~cm}^{-1}$ with $J "=16(\mathrm{~b})$. The results highlight the importance of using more advanced lineshape models for high- $J \mathrm{H}_{2} \mathrm{O}$ transitions influenced by line-narrowing processes. Figures adapted from [46].

\subsubsection{Dicke Narrowing and the Rautian and Galatry Profiles}

At number densities near STP, Dicke narrowing may lead to a narrowed lineshape, typically observed as gullwing residuals between a measured spectrum and its best-fit Voigt lineshape (see Figure 4a). Dicke narrowing [52] refers to a collision-induced reduction of the Doppler width (compared to that predicted by Eq. 10) that occurs when collisions confine the translational motion of the absorbers. It is most noticeable for high $J$ transitions of molecules with large rotational-energylevel spacing (e.g., $\mathrm{H}_{2} \mathrm{O}, \mathrm{HCl}, \mathrm{HF}$ ), since collisionalbroadening of such transitions is relatively weak, and is usually more pronounced when the mass of the collision partner is much greater than that of the absorbese

The Rautian [53] (RP) and Galatry [54] (GP) profiles address Dicke narrowing using hard- and soft-collision models, respectively, via a parameter, $z$, that is related to the frequency of velocity-changing collisions, $\beta$, according to:

$$
z=P \sum_{k} \chi_{k} \beta_{k}(T) / \Delta \nu_{D}^{\prime}
$$

Here, $\Delta \nu_{D}^{\prime}=\Delta \nu_{D} / 2 \sqrt{\ln 2}$ is the $1 / e$ Doppler HWHM. While $\beta$ is lineshape specific, due to the use of different collision models, it has been shown that the Rautian Profile is nearly identical to the Galatry Profile when $\beta_{\text {Rautian }}=3 / 4 \beta_{\text {Galatry }}$ (see [55] and Figure 4a). Similar to the collisional-broadening coefficient, the temperature dependence of $\beta_{k}$ is typically modeled using the power law. When $\beta=0$, the Rautian and Galatry profiles both reduce to the Voigt profile. 


\subsubsection{Speed-Dependent Collisional Broadening and the Speed-Dependent Voigt Profile}

${ }_{525} \quad$ Like Dicke narrowing, speed-dependent collisional broadening tends to narrow the absorption lineshape leading to gull-wing shaped residuals (when compared to the Voigt profile, see Fig. 4a) and is also most noticeable for high- $J$ transitions of molecules with 530 large rotational-energy-level spacing at number densities near STP. Speed-dependent collisional broadening (i.e., heterogenous or non-Lorentzian collisional broadening) refers to cases where the efficiency of collisional broadening depends on the absorber's speed. Rigorabsorber's speed alters the amount of kinetic energy available for promoting rotationally inelastic collisions and altering the trajectory of collisions; however, ignoring this complexity is surprisingly accurate in the ty of cases relevant to

Several lineshape models have been developed to address speed-dependent broadening (see [55] and the references therein), among the most popular is the quadratic-speed-dependent Voigt profile (qSDVP) [56, 57, 58] which addresses speed-dependent collisional broadening via a speed-dependent collisionalbroadening coefficient, $\gamma_{k, 2}$, and corresponding collisional FWHM, $\Delta \nu_{c, 2}$. In the event that $\Delta \nu_{c, 2}=$ $\gamma_{k, 2}=0$ (i.e., the speed dependence of broadening is 50 negligible), the speed-dependent Voigt profile reduces to the standard Voigt profile. Currently, very little temperature-dependent experimental data exists for, $\gamma_{k, 2}$, however the power-law model has been suggested [55] and demonstrated [46] (see Figure 5) as a suitable model for the temperature-dependence of $\gamma_{k, 2}$, albeit over only a moderate sized temperature range.

\subsubsection{The Impact Approximation and the $\chi$-Function}

At high number densities, the impact approximation can breakdown leading to elevated absorption in the transition wings (compared to that predicted by Lorentzian-based lineshape models, see Figure 6). The impact approximation assumes that collisions are $i^{555}$ stantaneous. Hartmann et al. [59] suggests that the impact approximation is valid when $2 \pi c\left|\nu-\nu_{o}\right| t_{c o l l}<<$

Table 2: Collisional-broadening and -narrowing parameters 1590 quired for different lineshape models.

\begin{tabular}{ccccc}
\hline Lineshape & $\nu_{D}$ & $\beta$ & $\nu_{c}$ & $\nu_{c, 2}$ \\
\hline Gaussian & $\mathrm{X}$ & & & \\
Lorentzian & & & $\mathrm{X}$ & \\
Voigt & $\mathrm{X}$ & & $\mathrm{X}$ & \\
Galatry & $\mathrm{X}$ & $\mathrm{X}$ & $\mathrm{X}$ & \\
Rautian & $\mathrm{X}$ & $\mathrm{X}$ & $\mathrm{X}$ & \\
qSD-Voigt & $\mathrm{X}$ & & $\mathrm{X}$ & $\mathrm{X}$ \\
\hline
\end{tabular}

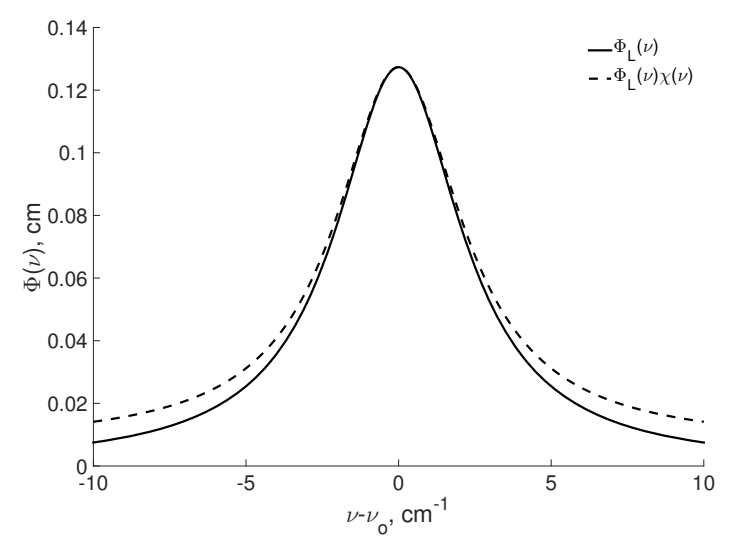

Figure 6: Simulated Lorentzian profile and that corrected with $\chi$-Function for an absorption transition with $\gamma=0.05 \mathrm{~cm}^{-1} / \mathrm{atm}$ at $50 \mathrm{~atm}$.

1 where the collision duration $t_{\text {coll }}=\frac{1}{\bar{g}} \sqrt{\frac{\Delta \nu_{c}}{n} \frac{c}{\bar{g}}}$ where $\bar{g}$ is the mean relative speed of collision partners. This corresponds to number densities $<\approx 5$ amagat, depending on species [59].

Several researchers have developed and investigated methods for addressing the breakdown of the impact approximation. The most widely used is the CKD model developed by Clough et al. [60] which uses an empirical frequency-dependent correction factor, known as the $\chi$-function, to correct the Lorentzian (or Voigt) lineshape according to $\phi(\nu)=\phi_{L}(\nu) \chi(\nu)$ where $\chi=1-\left(1-\chi^{\prime}\right) \frac{\left(\nu \pm \nu_{o}\right)^{2}}{25^{2}}$ for $\left|\nu \pm \nu_{o}\right| \leq 25 \mathrm{~cm}^{-1}$ and $\chi=\chi^{\prime}$ for $\left|\nu \pm \nu_{o}\right| \geq 25 \mathrm{~cm}^{-1}$ with $\chi^{\prime}=C \exp \left(-z^{2}\right)$ and $C=6.65$ and $z=\left|\nu \pm \nu_{o}\right| / 75$. However, several researchers have suggested $C$ is temperature dependent [59], and Rieker et al. [61] showed that $C=2.5$ was more accurate for $\mathrm{H}_{2} \mathrm{O}$ spectra near $7204 \mathrm{~cm}^{-1}$ at 700 $\mathrm{K}$.

\subsubsection{Line Mixing}

At high number densities, line mixing may also need to be accounted for to accurately model infrared absorption spectra. Line mixing (i.e., collisional coupling or collisional interference and not to be confused with line blending or overlapping) occurs when inelastic collisions transfer molecules between the two upper states and two lower-states of two absorption transitions. This process transfers absorption intensity (i.e., population) from the far wings of each transition into the region between transitions. In other words, collisions transfer absorbers from weakly absorbing regions to strongly absorbing regions leading to a narrowing of spectral structure [62] (see Figure 7). The absorption spectrum can no longer be described as the sum of that of individual transitions using Eq. 2 and models that account for this process are needed. Unlike Dicke nar- 

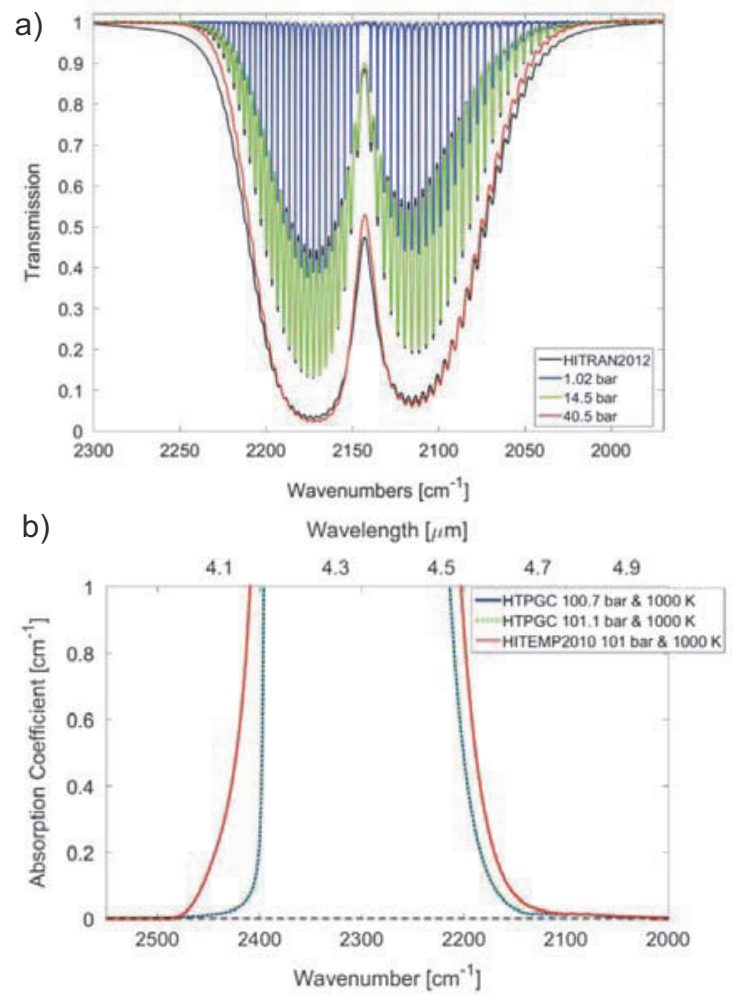

Figure 7: FTIR measurements of fundamental vibration bands of $1 \% \mathrm{CO}$ in $\mathrm{N}_{2}$ (a) and $5 \% \mathrm{CO}_{2}$ in $\mathrm{N}_{2}$ (b) compared witło simulated spectra calculated using HITRAN 2012 [1] and the Voigt Profile. Reprinted from [65] with permission from Elsevier.

rowing and speed-dependent broadening, line mixing is most pronounced when the spacing between rotation $\sigma^{6}-$ energy-levels is small such that molecules can easily be shuffled between levels. As a result, line mixing is much more problematic for $\mathrm{CO}_{2}$ and $\mathrm{CO}$ compared to $\mathrm{H}_{2} \mathrm{O}$.

605 For example, Farooq et al. [63] showed significant line mixing effects at only 10 amagat for $\mathrm{CO}_{2}$ transition near $2.7 \mu \mathrm{m}$, while Nagali [64] suggests that line mixing for $\mathrm{H}_{2} \mathrm{O}$ is not significant below 50 amagat using relations put forth by Hartmann et al. [59].

\subsubsection{Results and Discussion}

Numerous researchers $[66,67,68,69,70,58,71,72$, $73,74,46]$ have shown that using lineshape models that account for Dicke narrowing and/or speed-dependent collisional broadening at number densities comparable 615 to STP can reduce mildly inaccurate lineshape residwo als (i.e., $1-5 \%$ error) of the Voigt profile by $\approx 1$ order of magnitude, typically leading to residuals below $0.1 \%$ of the peak absorbance. While these modest gains in accuracy may seem insignificant from the standpoint of practical sensing applications in combustion applications, using such lineshape models (when appropriate) to develop an empirically derived collisionalbroadening database can translate into large gains in accuracy at higher pressures. For example, the recent study by Goldenstein et al. [74] showed that simulations performed using $\mathrm{N}_{2}$-broadening coefficients derived from least-squares fitting the Voigt profile to $\mathrm{H}_{2} \mathrm{O}$ spectra at low pressures (i.e., 0.1 to $1 \mathrm{~atm}$ ) overpredicted the peak absorbance of several high- $J \mathrm{H}_{2} \mathrm{O}$ lines in $\mathrm{N}_{2}$ by $\approx 30 \%$ at $15 \mathrm{~atm}$ and $1500 \mathrm{~K}$. In comparison, simulations performed using $\mathrm{N}_{2}$-broadening coefficients derived from Galatry-profile fits to low-pressure spectra matched the measured spectra at 15 atm within $5 \%$ of the peak absorbance. This being said, it is particularly important to use more sophisticated lineshape profiles when the best-fit parameters will be used to simulate spectra at temperatures and pressures outside the domain for which they were obtained.

Figure 4a shows simulated lineshapes of the $\mathrm{H}_{2} \mathrm{O}$ transition near $7471.6 \mathrm{~cm}^{-1}$ using empirically derived lineshape parameters for Voigt, Rautian, Galatry, and speed-dependent Voigt profiles. The more advanced lineshape profiles exhibit a strikingly similar shape which leads to controversy regarding the underlying physics that is primarily responsible for the gull-wing residuals of the Voigt profile (Dicke narrowing or speeddependent broadening). However, a growing body of literature (see [46] and the references therein) showing transition-specific Dicke narrowing parameters suggests that speed-dependent broadening may be the primary mechanism, since Dicke narrowing is a speciesspecific process and should not be transition-specific [73]. The recent study by Goldenstein and Hanson [46] shows that the speed-dependent Voigt profile yields similar improvements at high temperatures, however, with best-fit lineshape parameters that exhibit a much more physical behavior than those of the Rautian and Galatry profiles, further supporting this theory that the speed-dependent Voigt profile is a more physical model. Regardless, further studies over a broad range of temperatures and pressures are needed to confirm this theory, and if possible, lineshape models that account for both Dicke narrowing and speed-dependent broadening need to be studied.

Several researchers have studied the influence of the impact approximation and line mixing on IRabsorption spectra of $\mathrm{H}_{2} \mathrm{O}[59,75,64,61]$ and $\mathrm{CO}_{2}$ $[76,77,63,78,65]$, and some studies have been conducted at high temperatures [76, 59, 75, 64, 61, 78, 65]. Regarding $\mathrm{H}_{2} \mathrm{O}$, Nagali and Hanson [75] acquired TDLAS measurements of $\mathrm{H}_{2} \mathrm{O}$ absorption spectra near $7117 \mathrm{~cm}^{-1}$ in an $\mathrm{N}_{2}$ bath at number densities up to 18 amagat. The authors found that simulations performed with the Voigt profile and additive approximation agreed with measured spectra (within an uncertainty of $\approx 5 \%$ ) in a $2 \mathrm{~cm}^{-1}$ region around the transition linecenter. Later these authors investigated the transition near $7185 \mathrm{~cm}^{-1}$ at similar conditions, and found 
that if the $\chi$-Function was used to model the wings of 680 a strong neighboring transition, simulations performed with the Voigt profile agreed with measurements. As result, the authors concluded that line mixing was not significant (for the $\mathrm{H}_{2} \mathrm{O}$ transitions studied) at up to 18 amagat. Furthermore, Nagali [64] later estimated that line-mixing is not significant for $\mathrm{H}_{2} \mathrm{O}$ below 50 amagat using the relations put forth by Hartmann [5a] who states that the spacing between strongly coupled $\mathrm{H}_{2} \mathrm{O}$ transitions in the $\mathrm{P}$ and $\mathrm{R}$-branch is typically 10 $\mathrm{cm}^{-1}$. However, experimental results for a wide range of transitions remain needed to validate this prediction.

In comparison to $\mathrm{H}_{2} \mathrm{O}$, line mixing is much mozes significant for $\mathrm{CO}_{2}$. Farooq et al. [63] acquired FTIR measurements of the $\nu_{1}+\nu_{3} \mathrm{CO}_{2}$ band near $2.7 \mu \mathrm{m}$ at room temperature and up to 30 bar, and showed that a spectroscopic model developed by Niro et al. [79] (which accounts for line mixing) significantly inbo proved the accuracy of the modeled spectra in the band center and wings compared to that calculated using the Lorentzian and $\chi$-Function. More recently, Chris700 tiansen et al. [65] acquired FTIR measurements of $\mathrm{CO}_{2}$ absorption near 2, 2.7 and $4.2 \mu \mathrm{m}$ at high-temperaturess and -pressures (see Figure 7). Measured spectra of the $\nu_{3}$ fundamental band at 100 bar and $1000 \mathrm{~K}$ are reported, and simulations with the Voigt profile and AN2012 database 11] were shown to significantly overpredict the absorption in the band wings, perhapos due to line-mixing effects. In addition, Christiansen et al. [65] show similar results for the $\mathrm{CO}$ fundamental vibration band at 40.5 bar and $296 \mathrm{~K}$. Several authors modeling of high-pressure $\mathrm{CO}_{2}$ absorption spectra beyond that of the Voigt profile [76, 80, 81, 82, 83], however, significant experimental research remains needed to evaluate the accuracy of these models.

\subsection{Line-by-line data}

For relatively small combustion species, with approximately 8 atoms or less, spectroscopic parameters in the infrared are mostly catalogued on a line-by-line basis by a few well-established databases, namely HITRAN/HITEMP [1, 47], GEISA [85], and CDSD [867 with the latter database for carbon dioxide only. These databases are updated every few years and combine data collected from numerous laboratories with the${ }_{22}$ oretical models to compile parameters for collisional broadening, intensity/linestrength, and pressure shifst used, amongst other purposes, for predictive modeling of absorption spectra as described in the previous section. Pressure broadening and shift parameters are 730 generally limited to collisions with nitrogen, air, or the absorbing species (i.e., self). The line-by-line databases cover most products and intermediates of combustion due to their small size, but the number of lines within the databases for each species varies considerably (e.g. $\approx 170,000$ lines for $\mathrm{CO}_{2}, \approx 12,000$ lines for $\mathrm{C}_{2} \mathrm{H}_{2}$ in $\mathrm{HI}-$ TRAN) based on the relative number of active vibrational modes, the linestrength cutoff implemented in models, and the degree of investigation.

It should be noted that most spectroscopic databases have been constructed with low-temperature data and applications (e.g., atmospheric sensing) in mind, and combustion scientists must be cautious when utilizing these basic data for high-temperature simulations. Limitations of spectral modeling at combustion temperatures using data acquired at lower temperatures is largely due to insufficiency of the power law for modeling the temperature-dependence of collisionalbroadening and pressure-shift as mentioned in Section 3.1.2. The Carbon Dioxide Spectroscopic Databank (CDSD) and HITEMP are notable exceptions, with line lists for five key combustion species $\left(\mathrm{H}_{2} \mathrm{O}\right.$, $\left.\mathrm{CO}_{2}, \mathrm{CO}, \mathrm{NO}, \mathrm{OH}\right)$ created to more accurately represent high-temperature spectra at $1000 \mathrm{~K}$ or above. While managed independently, the current version of HITEMP (2010) incorporates parameters of CDSD1000 [86]. More recently, the CDSD, managed by Tashkun et al, released CDSD-4000 [87], a line list developed for the temperature range of 2500 to $5000 \mathrm{~K}$. Another high-temperature, species-specific line list is the BT2 water database [88] developed by Barber et al., which is also incorporated in HITEMP.

\subsection{Cross-section data}

For larger combustion species, namely fuels and larger fuel intermediates $(\approx \mathrm{C} 3$ or higher $)$, the high number of vibrational modes and corresponding infrared-active bands present a more daunting spectra to model on a line-by-line basis. This is exacerbated by the increased difficulty to experimentally investigate individual rovibrational transitions due to the more crowded and blended spectra, even at very low pressures. As such, spectroscopic data for these larger molecules are more commonly documented in a cross-section format at a given condition (temperature, pressure, composition). The absorption cross-section is most often resolved as a function of wavelength using a Fourier-Transform Infrared (FTIR) spectrometer. Two of the most comprehensive efforts to collect and aggregate infrared cross-section data have been led by the Pacific Northwest National Lab (PNNL) [89] and the National Institute of Standard and Technology (NIST) [90] with each database containing hundreds of species and thousands of spectra. The majority of these spectra are collected at or very near room temperature, with the PNNL database offering spectra up to $50 \mathrm{C}$, and use nitrogen as the balance gas for various concentrations of the absorber. Due to the limited tempera- 

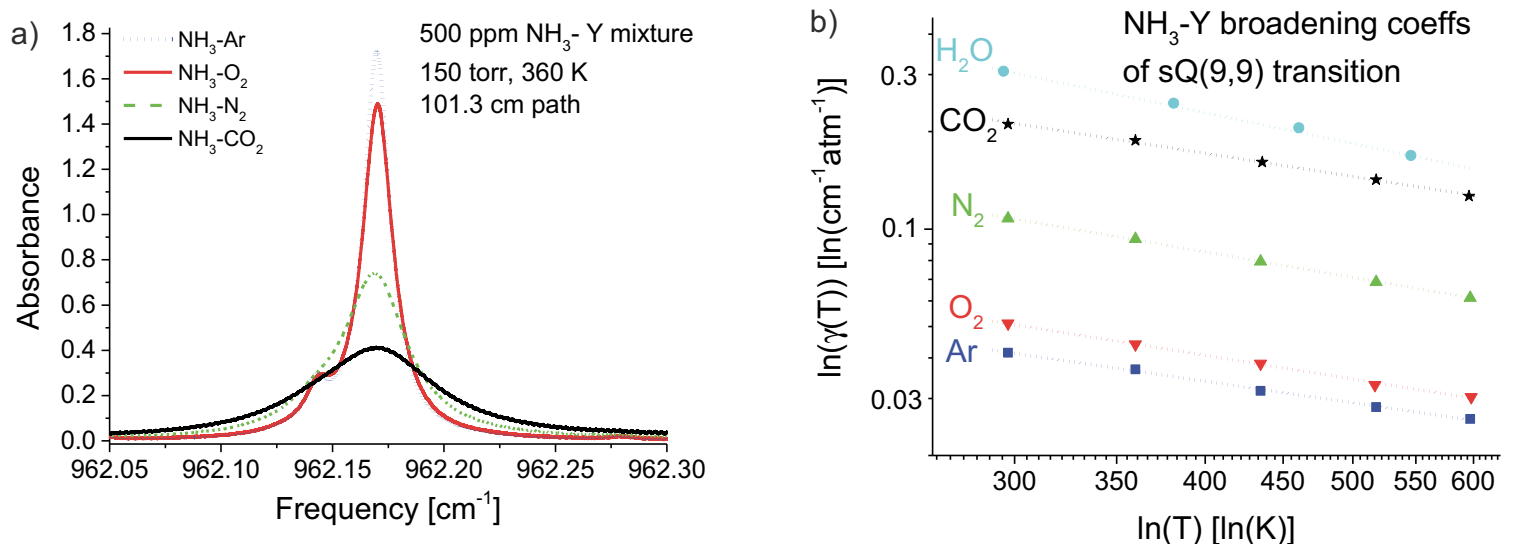

Figure 8: Measured absorption spectra of $\mathrm{NH}_{3}$ near $962.17 \mathrm{~cm}^{-1}$ for different bath gases (a) and corresponding best-fit collisionalbroadening coefficients of bath gas as a function of temperature (b). Results taken from Sur et al. [84].

ture range of the cross-section data in PNNL and NIST29 the spectra for various fuels of interest to combustion must be cautiously used with regards to wavelength seanother option, however, is to use uncatalogued data published by a wide range of researchers, some of whick is discussed in the next section.

\subsection{Uncatalogued data}

Efforts to aggregate and standardize the format of spectroscopic data on a line-by-line or cross-section bæe sis inherently require limitations of scope that leave an enormous amount of published spectroscopic data without centralized access. A significant portion of this 300 uncatalogued data has been produced for combustion sensing purposes and falls into two primary categoriess (1) high-temperature data, and (2) data collected with combustion species (e.g. $\mathrm{CO}_{2}, \mathrm{H}_{2} \mathrm{O}$ ) as bath gas. The difficulty with extrapolating low-temperature spectroto elevated temperatures drives the need for hightemperature datasets, while highly variable speciesspecific pressure broadening motivates the latter. Figure 8, reprinted from Sur et al. [84], highlights the impact of balance gas composition on the observed absorption spectra for $\mathrm{NH}_{3}$ at slightly elevated temperatures, representative of combustion exhaust. $\mathrm{CO}_{2}$ and $\mathrm{H}_{2} \mathrm{O}$ have been observed to have collisional broadeas ing coefficients that are much more than twice that ${ }^{315}$ of nitrogen or air, such that a gas mixture with only perhaps $5-10 \%$ of these combustion species can significantly alter the lineshape and peak absorption for a given transition. While documenting all such releva畹 but uncatalogued infrared data is beyond the scope of this paper, we note some recent contributions (either at high temperature or with combustion species as balance gas) that have been made for a number of molecules found in combustion flows including: $\mathrm{H}_{2} \mathrm{O}$ [91, 92, 88, 93, 94, 74, 46, 95, 96], $\mathrm{CO}_{2}[97,98,99,100]$, $\mathrm{CO}[101,102,103], \mathrm{CH}_{4}[104,105,106], \mathrm{C}_{2} \mathrm{H}_{4}$ [107], $\mathrm{C}_{3} \mathrm{H}_{6}$ [108, 109], $\mathrm{iC}_{4} \mathrm{H}_{8}$ [110], NO [111], $\mathrm{NO}_{2}$ [112], and $\mathrm{NH}_{3}[84,113,114,115]$. In addition, temperature, pressure-, and bath-gas-dependent cross sections for a large number of hydrocarbons at elevated temperatures have been published [116, 117, 108, 118, 119]. It should be noted that recent efforts have been taken to integrate and centralize spectroscopic data at the simulation and visualization level such that many datatypes and databases (line-by-line, cross-section, etc.) may be readily utilized together for spectral modeling [120]. This approach provides for opportunity to integrate current uncatalogued data and future data that may not fit into the more focused scope of existing databases.

\section{Techniques}

This section discusses the fundamentals of the most common LAS techniques; recent applications of these techniques are discussed in Sections 7 through 13. Specifically, two categories of LAS techniques will be discussed: direct absorption (DA) and wavelengthmodulation spectroscopy (WMS) and these techniques will be further broken into the subcategories: fixed wavelength or scanned wavelength. In the former, the laser's wavelength is resonant with an absorption transition of interest and constant in time (for DA) or sinusoidally modulated about a fixed wavelength (for 

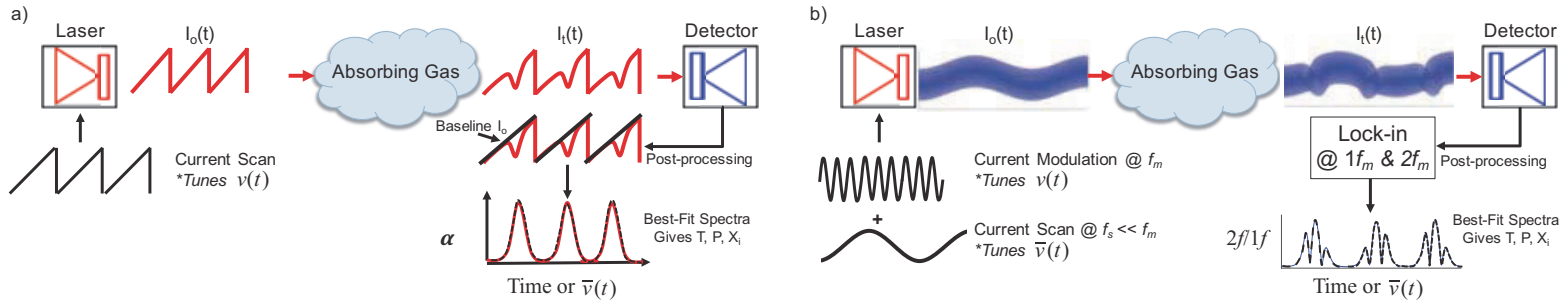

Figure 9: Schematics for scanned-wavelength direct absorption (a) and scanned-WMS (b).

WMS). In the latter, the laser's wavelength is scanned across a portion of the absorption spectrum of inteds est, and in WMS, this wavelength scanning is accompanied by simultaneous sinusoidal wavelength modulation performed at a much higher frequency (typically 50 to 100 times greater than scan rate). In addition, a variety of hyperspectral direct-absorption techniques have emerged in the last 15 years and, due to their unique abilities, will be discussed separately from conventional scanned-wavelength DA.

Fixed-wavelength techniques are inherently less robust due to their limited spectral information and, as a result, are typically only used when extreme time reso ${ }_{865}$ olution (order of $\mathrm{MHz}$ ) is required and non-absorbing transmission losses are negligible or can be corrected for (e.g., with use of a non-resonant laser [121]). Fixedwavelength direct absorption has been used extensively and effectively in shock-tube studies of chemical kinetics $[3,122]$, and some work has been done in IC-engines [123] and pulse-detonation combustors [121, 104].

Currently, the majority of laser-absorption sensor development for combustion flows revolves around advancing the state of scanned-wavelength techniques due 875 to their 1) increased robustness and versatility, 2) $\mathrm{in}^{915}$ creased potential for monitoring practical combustion systems, and consequently, 3) their increased complexity. As a result, this section will focus on describing the fundamentals of the various LAS techniques, as well as,

\subsection{Scanned-Wavelength Direct-Absorption Spec- troscopy}

Scanned-wavelength direct absorption (scanned-DA2) was first applied to combustion gases in the late ity of its data interpretation, continues to be widely used to study combustion when SNR-levels permit $[124,102,125,126]$. Modern scanned-DA is typicalłyo performed with distributed-feedback diode, interband-

${ }_{890}$, or quantum-cascade lasers. In this case, the laser's wavelength is scanned (via injection-current tuning) across one or a few absorption transitions to resolve the absorption spectrum of interest (see Figure 9aops
Gas properties are then calculated from the absorbance at a given wavelength or, more typically, from the integrated absorbance using Eq. 2 or 4, respectively. In harsh combustion environments, scanned-DA can suffer from low SNR, particularly in cases of lowabsorbance $(\alpha<0.001)$ or at high pressures $(>5$ to 10 atm) where individual transitions cannot be resolved. To overcome this, numerous researchers have developed more advanced diagnostic techniques (e.g., WMS, Hyperspectral-DA) discussed in the following subsections.

\subsection{Hyperspectral Direct-Absorption Spectroscopy}

Strictly speaking, scanned-DA is typically used to describe cases where the laser's wavelength/frequency is tuned over a narrow spectral range $(\approx 0.1$ to $10 \mathrm{~cm}^{-1}$ ) to resolve one or several absorption transitions. Here, hyperspectral direct-absorption spectroscopy (hyperspectral-DA) will be used to refer to all direct-absorption techniques spanning a region of the spectrum greater than $10 \mathrm{~cm}^{-1}$; further distinction will only be provided when necessary. Such techniques have been referred to as wavelength-agile DA [36, 127], swept-wavelength DA [38], and supercontinuum laserabsorption [128], typically to indicate differences in the type of light source used and these differences are discussed in detail in Sect. 6. Regardless of these differences, all hyperspectral-DA techniques are used to measure a large portion of an absorption band, typically at very high rates (order of 50 to $100 \mathrm{kHz}$ ) and with a relatively broad linewidth (on the order of 0.1 $\mathrm{cm}^{-1}$ ). This unique combination of features has mandated the use of a variety of unique data processing and spectral-fitting techniques to overcome challenges associated with large datasets and the need to accurately model significant portions of an absorption band convoluted with an estimated instrument function. Despite these complexities, hyperspectral-DA techniques have demonstrated the ability to overcome most of the challenges limiting conventional scanned-DA, and as a result, have emerged as one of the most promising LAS technologies for characterizing harsh combustion environments. 
Fixed-WMS

W
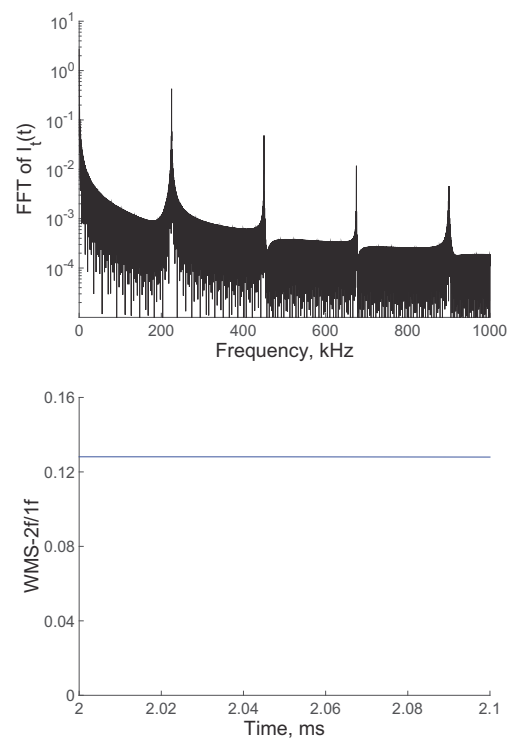

Peak-Picking Scanned-WMS
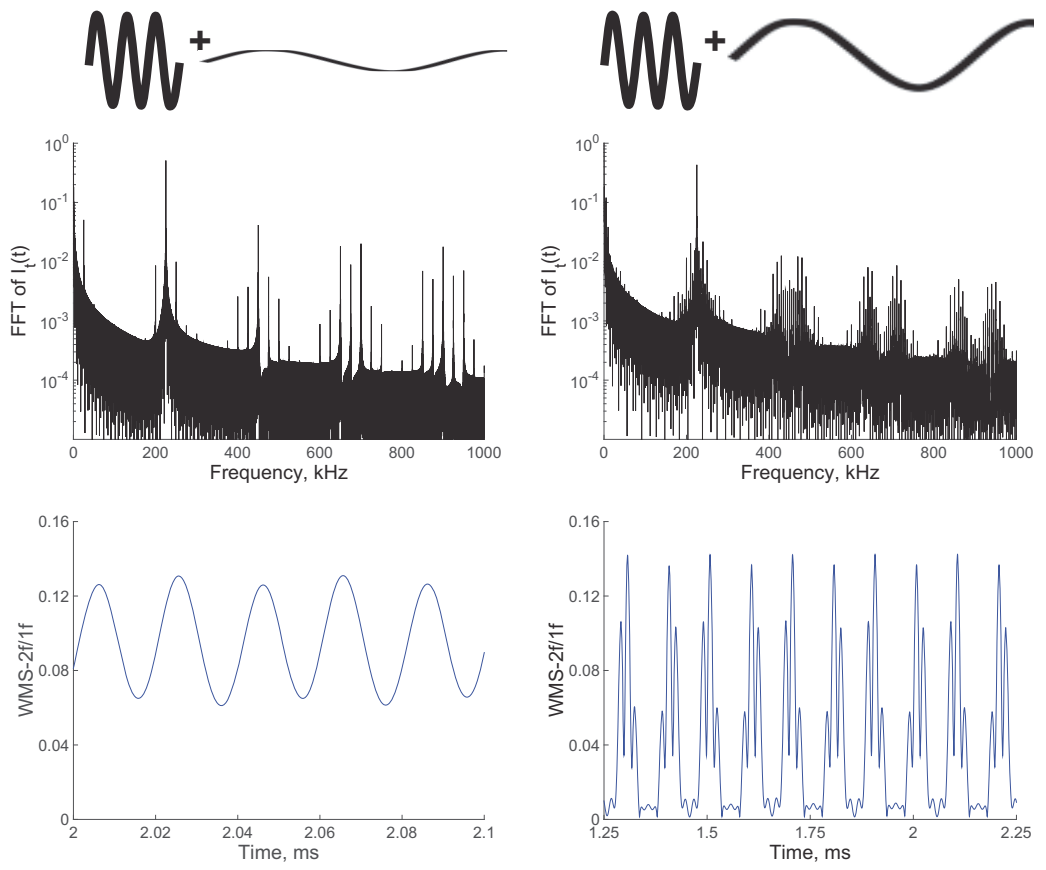

Scanned-WMS
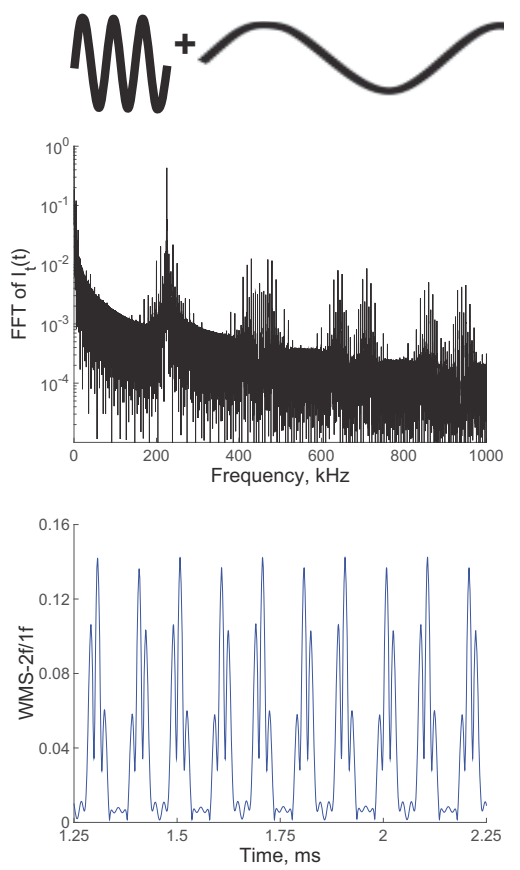

Figure 10: FFT of transmitted light intensity for a fixed-WMS, peak-picking scanned-WMS, and scanned-WMS experiment interrogating the $\mathrm{H}_{2} \mathrm{O}$ absorption transition near $7185.59 \mathrm{~cm}^{-1}$ and corresponding WMS- $2 f / 1 f$ time-histories for a diode laser modulated at $200 \mathrm{kHz}$. The scan frequency for the peak-picking scanned-WMS figures is $25 \mathrm{kHz}$ and that for the scanned-WMS figures is 5 $\mathrm{kHz}$.

\subsection{Wavelength-Modulation Spectroscopy}

Wavelength- and frequency-modulation spectroscopy were first developed in the early 1980s [20] to reduce the influence of laser and detector 940 noise, thereby improving measurement fidelity in gas-sensing applications. Since then, wavelengtbes modulation spectroscopy (WMS) has been used extensively to study a wide range of harsh combustion environments $[3,129,39,27]$ particularly due to several experimental and theoretical advancements enabling robust, calibration-free WMS techniques [130, 40, 41, 131, 132, 133]. Typically, the first- and second-harmonic signals are used to calculate gas properties and will be the focus here. However it should be noted that using higher harmonics ( $3 f, 4 f$, etc.) has been shown to exhibit some unique advantagess as discussed in Section 4.5.4 and [134, 135, 136, 137]. Several variants of WMS have been used and will be discussed here, namely: fixed-WMS, peak-picking 955 scanned-WMS, and scanned-WMS. A schematic illustrating scanned-WMS-2 $f / 1 f$ is shown in Fig. 96 and Figure 10 illustrates how the WMS-2f/1f signals associated with each of the aforementioned techniques differ in the Fourier and time domain. A detailed discussion of these differences can be found in [138].

In fixed-WMS the laser's wavelength is sinusoidally modulated (typically via injection current modulation) about a given location on an absorption feature of interest. The modulation shifts absorption information to the harmonics of the modulation frequency, $f$, and the harmonic signals ( $1 f, 2 f, 3 f$ etc.) can be extracted from the raw detector signal using digital lock-in filters during post-processing [41] or using analog lock-in amplifiers prior to data acquisition. In this case, the lock-in filter bandwidth defines the theoretical sensor bandwidth. By modulating at high frequencies ( $f$ typically from $10 \mathrm{kHz}$ to $1 \mathrm{MHz}$ ), the harmonic signals are elevated above lower-frequency noise sources (e.g., electronic, environmental) which can improve their SNR [139]. Cassidy and Reid [130] first showed that when WMS is performed using injection-current tuned lasers (e.g., diode lasers, quantum-cascade lasers), the higher harmonics can be normalized by the $1 f$ signal to remove their dependence on the DC light intensity impinging on the detector. This attribute enables WMS$n f / 1 f$ signals that are immune to intensity variations (e.g., from beam steering or window fouling or other non-absorption losses) that vary in time slowly com- 
pared to $f$.

985 ulation is accompanied by a small-amplitude wavelength scan to resolve the peak of the WMS- $2 f / 1 f$ signal near linecenter, thereby providing a known wavelength reference. This improves measurement robustdrift) while minimizing the additional frequency content centered around each harmonic, thereby reducing potential for cross talk and facilitating frequency multiplexing of multiple lasers. In this case, only the value 95 of the WMS- $2 f / 1 f$ signal near the transition linecenters is used to calculate gas properties (see [138]) and the measurement bandwidth is set by the scan frequency.

In scanned-WMS the nominal wavelength of the laser is scanned across an absorption transition to extract WMS spectra, thereby enabling spectral fitting and rose moving the need for a priori knowledge of the transition line broadening [132]. In this case, gas properties can be extracted from measured WMS spectra analogous to scanned-wavelength direct absorption using the spectral-fitting technique developed by Goldenstein 10st al. [132] (later extended by Qu et al. [133] to include more laser parameters). In this method, WMS spectra are simulated by passing the simulated laser-intensity time-history $\left(I_{t}(t)\right.$, given by Eq. 15) through digital 1010 lock-in filters to extract the WMS spectra of interesso (e.g., WMS- $1 f,-2 f$, etc.).

$$
I_{t}(t)=I_{o}(t) \exp \left[-\sum_{j} S_{j}(T) P \chi_{A b s} \phi(\nu(t), T, P, \chi) L\right]
$$

Here $I_{o}(t)$ and $\nu(t)$ are given by a summation of sinusoids describing the laser's intensity- and wavelengthtuning response to the sinewaves applied to its injec1015 tion current (see [132]). It is important to note that modern calibration-free WMS models are applicable to all optical depths [40, 41, 131, 132, 133], however only the most recent $[131,132,133]$ accurately account for the laser intensity varying during injectioncurrent scanning (performed to scan the laser's nominal wavelength across the absorption transition of interest). This enables gas properties to be calculated from WMS spectral-fitting routines [132] (analogous to scanned-wavelength direct absorption), thereby removing the need for a priori knowledge of line broadening. It should be noted that methods for recovering absorption transition lineshapes have also been developed using WMS- $1 f$ signals [140, 141]; however, this approach requires use of non-resonant wavelengths (analogous to

\subsection{Cavity-Enhanced Techniques}

The previous sections discussed absorption techniques primarily aimed at improving sensor accuracy and robustness by rejecting signal noise or increasing the amount of useful information extracted by the sensor. However, in reality the sensitivity of all absorption measurements is fundamentally limited by the strength of the interrogated absorption transition(s) and/or the path length over which light interacts with the absorbers. The former is dictated by the physics of target species (i.e., Einstein-A coefficient, degeneracy, and Boltzmann statistics), however, the latter can be manipulated by the experimentalist by conducting the measurement in an optical cavity. This section will discuss a few of the most useful cavity-enhanced LAS techniques for increasing the sensitivity of LAS sensors.

There are three primary cavity-enhanced LAS techniques applicable to combustion applications: (1) intracavity-absorption spectroscopy, (2) cavity-ring down spectroscopy (CRDS), and (3) cavity-enhanced absorption spectroscopy (CEAS) or integrated-cavity output spectroscopy (ICOS). In intracavity-absorption spectroscopy the test gas is located within the laser cavity. Absorption transitions within the gain curve of the laser are sensitively detected by measuring the reduction in gain at these specific wavelengths. There is a long (40+ years) history of intracavity absorption using broadband laser sources (e.g., broadband dye lasers or relatively broadband gas lasers) and the application to combustion diagnostics has long been recognized, as such, we refer the reader to an excellent article by Harris [142], as well as a few recent papers employing fiber lasers $[143,144]$.

Figure 11 illustrates the operating principles of CRDS and CEAS. Typically in CRDS, pulsed laser light is injected on-axis into an optical cavity (formed by two dielectric coated mirrors) and the light intensity exiting the cavity is measured to determine the characteristic decay time $(\tau)$. In the limit of no scattering losses, the decay time is dictated by the reflectivity of the cavity mirrors and the single-pass absorbance $\left(\alpha_{S P}\right)$ given by Eq. 2 . With the mirror reflectivity known (e.g., from a measurement in the empty cavity), Eq. 16 can be inverted to solve for $\alpha_{S P}$ and, thus, gas properties.

$$
\begin{aligned}
I_{t}(t) & =I_{o} \exp (-t / \tau) \\
& =I_{o} \exp \left(-\left((1-R)+\alpha_{S P}(\nu)\right) c t / L\right)
\end{aligned}
$$

CRDS with high-finesse (i.e., high-reflectivity) cavities can increase the optical path by $10^{4}$ or more, thereby enabling highly sensitive measurements of trace species. However, these cavities suffer from very small transmission (typically requiring mode-locking with the TEM00 cavity-mode) and long residence times of the light which limits their time response. Furthermore, high-finesse cavities are highly susceptible to window fouling (e.g., from dust or soot) and require 

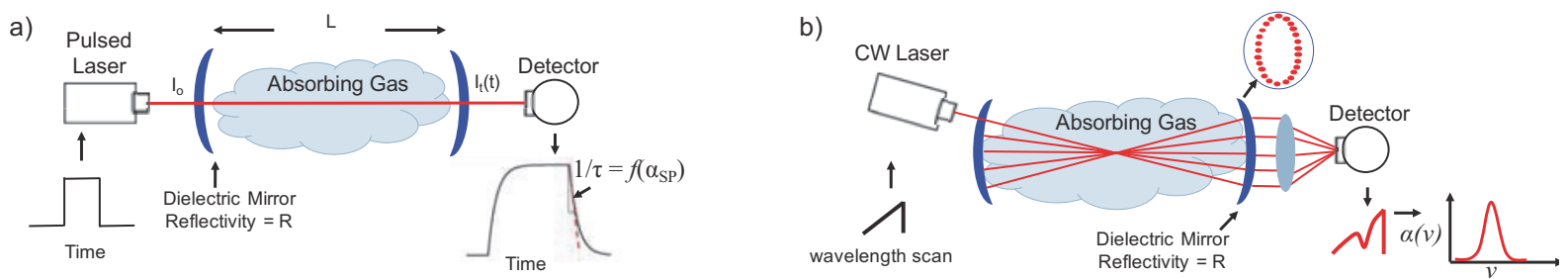

Figure 11: Schematics for cavity ringdown spectroscopy (a) and cavity enhanced absorption spectroscopy (b). Figures adapted from [11].

extreme care to maintain high mirror reflectivity.

In comparison, in CEAS continuous-wave laser light is typically injected off-axis into an optical cavity and the intensity of light exiting the cavity is measured on a photodetector. By using off-axis injection the reflecs tions are separated in space as they move around the perimeter of each mirror and many roundtrips are required before the light retraces its initial trajectory and overlaps upon itself. This leads to an effective cavity length on the order of $m$ to $\mathrm{km}$ and, therefore, a freespectral range that is comparable to the laser linewidth and is much smaller than the absorption transition HWHM. As a result, scanned-wavelength techniques can be employed to measure absorption spectra and the non-absorbing baseline. Gas properties are calculated using Eq. 17 (an analogue of Beer's Law) and a spectroscopic model.

$$
\alpha_{C E A S}(\nu)=-\ln \left(\frac{I_{t}}{I_{o}}\right)=\ln \left(1+G \alpha_{S P}(\nu)\right)
$$

Here, $\alpha_{C E A S}$ is the measured, cavity-enhanced absoripro tion and $G$ is the cavity gain set by the mirror reflectivity (determined by CRDS or calibration).

CEAS has been used extensively in high-finesses configurations for trace-gas sensing $[145,146,147,148]$ and the use of WMS has also been explored [149], howevens like CRDS these methods suffer from long cavity residence times and susceptibility to mirror fouling. Recently, low finesse (gain of $100 \times$ in path length) cavities have been integrated into shock tubes to improve the measurement sensitivity of combustion species whilfe maintaining measurement rates $(\approx 100 \mathrm{kHz})$ sufficient to resolve reaction kinetics $[150,151]$ and these studies are highlighted in Section 7.1.

It should be noted that there is a long history of using intracavity laser absorption and CRDS for combustion diagnostics, especially to monitor the concentration of minor reactive species. Much of this work has focused on the distribution of radicals in low-pressure flames using transitions in the visible and ultra-violet to test models of flame chemistry. Since this work is beyond the scope of this paper (being outside the IR domain), we refer the interested reader to excellent reviews by Cheskis [152] and by Mercier and Desgroux [153] who have been pioneers of intracavity laser absorption and CRDS for combustion applications.

\subsection{Challenges and Solutions}

This section will discuss some of the most common challenges and current solutions pertaining to infrared LAS sensors in combustion environments.

\subsubsection{Beam Steering and Emission}

In harsh combustion environments the effective baseline $I_{o}$ may vary in time due to non-absorbing transmission losses (e.g., from window fouling, light scattering or beam steering) or fluctuations in background emission. If these losses or gains in light intensity are not properly accounted for or avoided, the gas properties inferred from the detector signal will be incorrect.

In direct absorption, these challenges are typically mitigated using rapid and/or broad wavelength scanning such that these confounding effects are constant during the measurement time and can be more accurately accounted for. For example, baseline emission levels can be recorded in-between pulses or scans, assumed constant during the measurement, and subtracted from the measured detector signal during the measurement time. In addition, non-absorbing transmission losses in $I_{o}$ can be accounted for if constant or near-constant during the measurement by floating the baseline intensity in spectral-fitting routines. Broad wavelength tuning can improve the robustness of the latter by increasing the amount of spectroscopic information relative to the "noise" (i.e., uncertainty in baseline).

In WMS, high-frequency modulation and $1 f$ normalization are used to reject background emission and non-absorbing variations in light intensity 

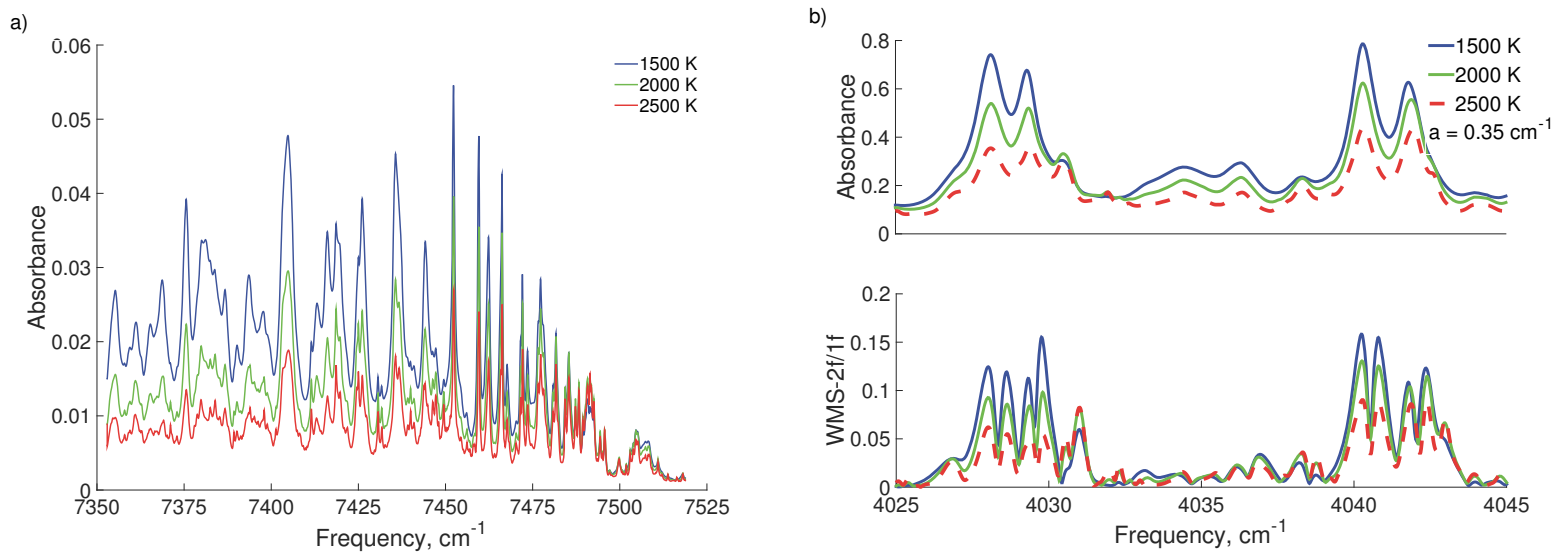

Figure 12: Simulated transmission spectra of MEMS VCSEL used for hyperspectral-DA in [154] (a) and WMS- $2 f / 1 f$ spectra near wavelengths used in [155] to measure $\mathrm{H}_{2} \mathrm{O}$ and temperature (b). Simulated spectra are for $10 \% \mathrm{H}_{2} \mathrm{O}, \mathrm{P}=25 \mathrm{bar}, \mathrm{L}=5 \mathrm{~cm}$, legend indicates temperature.

that vary slowly compared to $f$. As a result, measured WMS- $n f / 1 f$ spectra are inherently immune ito such transmission variations and no corrections are required during post-processing. However, despite $1 f$ normalization, variations in $I_{o}$ that occur at frequencies comparable to $f$ can introduce noise in the WMS

1165 signals. To reduce this noise one must improve the lighbt collection efficiency or modulate at higher frequencies to escape the frequency spectrum of the noise and/or increase the bandwidth over which $1 f$-normalization applies.

Several researchers have also developed strategies figor minimizing beam-steering effects via optical engineering. For example, Petersen [156] (and most recently Spearrin et al. [111]) found that using an integrating sphere can dramatically reduce beam-steering ef-

1175 fects in high-pressure gases and rigorous design rulere for maximizing light-collection efficiency are presented. Kranendonk et al. [157] developed a general design framework for minimizing the influence of beam steering without requiring a priori knowledge of the beam

1180 steering field. Whitney et al. [158] provides a de्s5 tailed analysis and discussion describing how to minimize beam-steering effects in sensors employing multimode fibers, and presents the design of a sensor using a fused-fiber lens and fiber-fused detector, the latter of which to minimize the influence of spatially nonuniform detector response. Most recently, Strand [159] showed that using an aspheric-lens doublet to collimate and focus (onto a detector) light exiting a multi-mode catch fiber can also dramatically improve tolerance to
1225

\subsubsection{Broad and Blended Spectra}

Broad and blended absorbance spectra complicate the determination of gas properties in three primary ways: 1) the absorption spectra at a given wavelength is governed by multiple transitions which have a unique dependence on gas conditions $(T, P, \chi), 2)$ nonabsorbing wavelengths may not exist nearby, complicating or precluding in situ determination of the nonabsorbing baseline, and 3) differential absorbance and WMS- $2 f$ signals are smaller.

The first complication has been addressed by several researchers by developing wavelength- and modulationdepth-selection routines that account for the influence of neighboring transitions $[160,161,162,155]$. For example, An et al. $[160,161]$ developed a quantity (the frequency-dependent lower-state energy) for quantifying the temperature sensitivity of a given wavelength and showed it could be used to identify the optimal wavelengths for thermometry. Goldenstein et al. [155] showed that by using wavelengths and specific modulation depths in regions of temperature-dependent spectral curvature, two-color WMS- $2 f / 1 f$ thermometry and mole fraction sensing could be performed using a single modulation depth over a range of 1000 to 3500 $\mathrm{K}$ and 5 to 50 bar (see Figure 12b).

The second complication only pertains to directabsorption techniques, and this challenge has been overcome by using an additional non-resonant light source (e.g., see [121, 104, 123, 163]) or broadly tunable light sources (e.g., see [33, 34, 164, 165, 154]). Figure 12a illustrates the wealth of spectral information that hyperspectral light sources can extract, enabling the baseline intensity to be floated in spectral-fitting routines. However, in the former approach it is critical to use non-resonant wavelengths as close to the resonant wavelengths as possible to minimize differences in wavelength-specific transmission losses (e.g., from droplet or particulate scattering) [121].

The last complication pertains to WMS, and this 
1230 challenge has been overcome by using large modulation depths $[166,42,167,162]$ and, most recently, by combining large modulation depths with strong midebs infrared absorption [97, 155, 46, 102]. WMS-1f sensing has also been demonstrated as an effective technique for sensing molecules with extremely broad spectra (e.g., large hydrocarbons and gasoline [168]). While WMS- $1 f$ is not immune to non-absorbing transmissiom losses, it is immune to background emission, as well as, laser and detector noise that exist outside the band1240 width of the lock-in filter used.

\subsubsection{Breakdown of the Impact Approximation}

As discussed previously in Section 4.5.3, at high number densities the impact approximation can breakdown, thereby introducing errors in spectroscopic mod1245 els that do not account for this. Since the impact approximation primarily effects the absorption transition wings, such errors can be mitigated by using wavelengths near linecenter. However, if multiple absorption transitions are overlapping (as is likely the case

1250 at number densities where the impact approximation breaks down), the contribution from neighboring transitions to the region near linecenter may be signifib cant. In this case, Rieker et al. [61, 77] showed that WMS-2 $f$ signal can be less susceptible to such errors since the contribution to differential absorbance (across the modulation depth) resulting from the far-wings of neighboring transitions can be small.

\subsubsection{Non-Zero WMS Background}

Real semiconductor lasers do not respond perfect担 linearly to injection-current tuning (i.e., sinusoidal current modulation results in intensity modulation with frequency components at $1 f, 2 f, 3 f$, etc.). As a result, the higher harmonic signals exhibit a non-zero background from higher-order intensity modulation (often

1265 referred to as residual amplitude modulation (RAM)). The RAM background decreases with increasing harmonic $[134,135,169,137]$, but increases with increasing modulation frequency and modulation depth [40]. $\mathrm{Li}$ et al. [40] showed that for telecommunications grade TDLs the second-order intensity modulation exhibits a quasi-quadratic dependence on the modulation depth, with a maximum value of $\approx 3 \%$ for $100 \%$ intensity modulation. However, it should be noted that we have found these results can vary substantially deo

1275 pending on the type of diode laser and manufacturer. To solve this issue, calibration-free WMS models that account for higher-order intensity modulation can be used $[40,41,131,132]$ or a measured intensity time history (in the absence of absorbers) can be used 16

1280 simulate WMS signals in the recently developed bruteforce calibration-free WMS models $[131,132]$. The latter approach can also be used to account for WMS background signals resulting from etalon effects in the beam path, and as a result, is currently the most accurate method if the etalons are stable. However, several researchers have shown that dithering of optical components [170], intelligent triggering timing [136], intelligent selection of the modulation depth [132], and the use of higher harmonics (e.g., $4 f$ ) [134, 135, 137] can all be used to reduce WMS background signals originating from etalon effects.

\subsubsection{1f-Normalization with Large Absorbance}

If normalization is optimal for, but not limited to, cases where the absorbance is small such that the $1 f$ signal is dominated by the laser's intensity modulation. As the peak absorbance increases, the $1 f$ signal near linecenter approaches zero. As a result, even small noise levels are magnified, leading to a WMS- $2 f / 1 f$ signal that can vary widely and erroneously near linecenter. This effect typically becomes problematic at absorbances greater than $\approx 0.5$. However, the $1 f$ signal away from linecenter typically remains sufficiently large for $1 f$ normalization. This can be exploited by normalizing the higher harmonic signals by the scan-averaged $1 f$ signal, as opposed to the local $1 f$ signal which, to the best of our knowledge, was first demonstrated in [131].

\section{Solutions for Nonuniform Flows}

Laser-absorption spectroscopy provides a pathintegrated measurement. In cases where the gas conditions vary along the measurement line-of-sight (LOS), the measured absorbance is given by Eq. 18 .

$$
\alpha(\nu)=\int_{0}^{L} \sum_{j} S_{j}(T) P \chi_{A b s} \phi_{j}(\nu, T, P, \chi) d l
$$

The path integral in Eq. 18 complicates the relationship between the measured absorbance and gas properties and a thorough discussion regarding the influence of non-uniform temperature, pressure, and composition can be found in [171]. Briefly, the measured absorbance is influenced by linear and non-linear dependences on pressure and absorbing-species mole fraction and a non-linear dependence on temperature through the transition linestrength and lineshape function. The non-linear dependencies can lead to the pathintegrated measurement deviating from path-averaged gas properties unless certain measures are taken [171].

Despite this complication several researchers have developed strategies to provide useful and quantitative measurements of gas properties in non-uniform gases $[172,173,32,174,175,176,177,178,179,180,181,182$, 
171]. This section will focus on discussing solutions ap1330 plicable to single-LOS measurements, however, a discussion of some of the most widely used and emerging tomographic LAS techniques will conclude this section.

\subsection{Single Line-of-Sight Solutions}

Several researchers have developed strategies to identify temperature non-uniformities $[174,178]$ and quantify the measurement uncertainty they induce [182]. Seitzman and Scully [174] developed a broadband sensor for monitoring temperature non-uniformity and $\mathrm{H}_{2} \mathrm{O}$ mole fraction in a gas-turbine combustor. By

1340 measuring the absorbance in at least three spectral windows with different temperature dependence, two temperatures can be calculated (one "cold" and onere "hot") with the difference between them quantifying how thermally non-uniform the flow is. A similar strat-

1345 egy was later used by Palaghita and Seitzman [177], Liu et al. [178], and Goldenstein et al. [138] using diode lasers to resolve individual absorption transitions fю̈ a simplified interpretation. Rieker et al. [43] built upon this technique by monitoring differences in the 1350 frequency content (from an FFT) of the "cold" and "hot" temperatures and showed that the measurements of fluctuations could be used to predict scramjet unbo start.

Strategies have also been developed to reduce sento non-uniform gas conditions $[173,174,175]$. For example, Ouyang and Varghese [173] developed a strategy for measuring absolute absorbing-species conbs centration in the presence of thermal and concentration boundary layers. This strategy relies on the use of transitions with specific values of lower-state energy such that the absorption measurement is insensitive to gases in the boundary layer, and relations areo put forth to identify suitable values of $E$ " for a given temperature of interest in the limit of small boundary

1365 layers. In their broadband absorption sensor, Seitzman and Scully [174] used a spectral window where the absorbance is nearly independent of temperature to measure $\mathrm{H}_{2} \mathrm{O}$ mole fraction with a maximum error of $8 \%$ for an unknown temperature (or temperature non-uniformity) from 1000 to $2100 \mathrm{~K}$.

Several LAS techniques have been developed to correct for or tolerate the large non-uniformities encounbe tered in flames $[172,171,183]$. Schoenung and Hanson [172] acquired thermocouple and line-of-sight ab-

1375 sorption measurements at multiple locations spanning the transverse (relative to the flow) axis of the flame to correct the path-integrated absorption measurement of $\mathrm{CO}$ assuming axisymmetric temperature and species distributions. Goldenstein et al. [171] developed a tweres color LAS strategy for measuring the absorbing-species column density, $N_{i}$, and absorbing-species-weighted path-averaged temperature, $\bar{T}_{n_{i}}$, given by Eq. 19 and
20, respectively, and showed that this technique is more accurate over a broad temperature range than a constant-linestrength approximation (similar to that used in [174]).

$$
\begin{gathered}
N_{i} \equiv \int_{0}^{L} n_{i} d l \\
\bar{T}_{n_{i}} \equiv \frac{\int_{0}^{L} T n_{i} d l}{\int_{0}^{L} n_{i} d l}
\end{gathered}
$$

This strategy requires use of absorption transitions with a linestrength that varies near linearly with temperature over the domain of the temperature nonuniformity. This can be achieved by using transitions with specific values of lower-state energy and is shown to be accurate over large temperature non-uniformities $(500$ to $1000 \mathrm{~K})$ at combustion temperatures (> 1000 $\mathrm{K})$. This strategy was later deployed to study flames in a scramjet combustor [184] and low-pressure burner [183].

Single-LOS strategies have also been developed to measure LOS distributions of temperature and composition [176, 179]. Sanders et al. [176] developed a strategy for recovering a discretized LOS distribution of temperature by scanning a VCSEL across 10 $\mathrm{O}_{2}$ A-band transitions with a unique temperature dependence. In this technique the LOS is discretized into bins ( 1 bin per absorption transition) and a system of linear equations is solved to determine the mole fraction and temperature in each bin. The discretized solution is then least-squares fit to a distribution function to provide a finer solution grid (provided that a representative distribution function is known a priori). The authors clarify that this technique works best when a distribution function can be assumed as the discretization technique alone may either be too coarse or illconditioned. Liu et al. [179] later built upon this technique by developing 1) a profile-fitting technique to solve for the defining parameters of a postulated temperature distribution profile and 2) a temperature binning technique to determine the probability density function of prescribed temperature bins along the LOS. These techniques were demonstrated in a twotemperature experiment using 5 fiber-coupled diode lasers targeting $\mathrm{H}_{2} \mathrm{O}$ absorption transitions.

\subsection{Laser-Absorption Tomography}

A wide range of laser-absorption-tomography (LAT) (a subclass of chemical species tomography (CST)) techniques have been developed and used to reconstruct $2 \mathrm{D}$ chemical-species and temperature fields in combustion flow fields $[185,186,187,124,188,189$, 190, 191, 192, 193], and several excellent reviews have 
been devoted to this subject $[194,195]$. In tomogra1430 phy, multiple lines-of-sight are used to sample a hetermo geneous measurement volume from unique viewpoints and the measured projections (i.e., path-integrated absorption) are used to reconstruct the 2D (or even 3D) field. In contrast to medical-imaging techniques employing 1000s of LOS (e.g., MRI, CT scan), LAT typas ically employs only 10 s to 100 s of LOS due to the optical-access limitations imposed by combustion applications. This sparse sampling precludes the use of the Fourier-transform-based algorithms (e.g., filtered 1440 backprojection) employed in medical imaging, and requires a distinct approach.

In most LAT applications, the $2 \mathrm{D}$ field is reconstructed from measured projections (i.e., the pathintegrated absorbance) by solving a linear systems of equations, $A x=b$, where the $m x n$ matrix $A$ specifies the known path length of each ray through each pixel, the $m$-vector $b$ contains the measured projections for each line-of-sight, and the $n$-vector $x$ specifies the unknown (i.e., to-be-solved for) absorbance or species-concentration in each pixel. A multitude of algorithms have been developed to solve for $x$ (e.g., in a least-squares sense or though minimization of an objective quantity) and address the ill-posed nature of this problem (i.e., multiple solutions often exist ${ }^{1500}$

1455 the solution is unstable and sensitive to input noise) [196, 197], including algebraic-reconstruction tomography (ART), simultaneous-ART (SART) [198], statistical image reconstruction (SIR) [199], and totalvariation-based compressed sensing (TVCS) [200, 201]. Daun et al. [195] points out that it is particularly important to distinguish between two cases in order to identify the most well-suited approach: 1) $A$ is discrete ill-posed [202] (e.g., when employing dense sampling of the tomography space) or, more likely in combustion 1465 applications, 2) the matrix equation is rank deficient (e.g., when employing sparse sampling) and, therefore, a priori information about the flow field is required to identify a unique solution.

Recently hyperspectral-tomography (HT) tech1470 niques have been developed and deployed to reconstruct 2D temperature and $\mathrm{H}_{2} \mathrm{O}$-concentration fields $[203,185,186,189]$. This method utilizes many absorption transitions, thereby adding increased spectral information to the reconstruction process. In practice, the temperature and species-concentration fields are reconstructed by minimizing the function:

$$
D\left(T^{r e c}, X^{r e c}\right)=\sum_{j=1}^{J} \sum_{i=1}^{I} \frac{\left[p_{m}\left(L_{j}, \lambda_{i}\right)-p_{c}\left(L_{j}, \lambda_{i}\right)\right]^{2}}{p_{m}\left(L_{j}, \lambda_{i}\right)^{2}} 1525
$$

where $p_{m}\left(L_{j}, \lambda_{i}\right)$ is the measured projection at location $L_{j}$ and a wavelength $\lambda_{i}, p_{c}\left(L_{j}, \lambda_{i}\right)$ is the corresponding projection calculated using the reconstructed $T$ and $X$ profile (denoted $T^{r e c}$ and $X^{r e c}$ ), and $J$ and $I$ are the total number of wavelengths and projection locations, respectively. For context, 12 unique wavelengths (chosen using the methods presented in [204]) were used for the jet-engine exhaust sensing presented in [189]. The authors state that this method has several advantages, including: 1) reducing the number of projections required for accurate tomographic reconstruction, 2) improved tolerance to noise, and 3) the ability to image temperature and species concentration simultaneously.

\section{Sources and Hardware}

This section will discuss the capabilities and limitations of both established and emerging light sources and hardware that are relevant to IR-LAS.

\subsection{Near-Infrared Hardware}

Historically, the majority of LAS sensors for combustion systems have operated in the near-infrared $(\approx 760$ to $\approx 2500 \mathrm{~nm}$ ) in order to leverage the robust and affordable hardware born out of the telecommunication industry in the 1990s. While the detection limit of these sensors is limited by the weaker absorption strength of the overtone and combination bands located in the near-IR, they have achieved widespread success, particularly in the detection of highly anharmonic absorbers (e.g., $\mathrm{H}_{2} \mathrm{O}, \mathrm{HF}, \mathrm{HCl}, \mathrm{NH}_{3}$ ) due to their greater absorption strength (only $\approx 10$ times less than their fundamental bands) and in flows with large quanitites of weaker absorbers or potential for long path-length measurements. This section will discuss some of the most widely used near-IR light sources and hardware to highlight their strengths and weaknesses.

\subsubsection{Distributed-Feedback (DFB) Tunable Diode Lasers (TDLs)}

DFB TDLs are one of the most widely used lasers due to their ease-of-operation, low cost, wavelength stability, rapid wavelength tunability, and ability to be manufactured over a wide range of custom wavelengths (760 nm to $3 \mu \mathrm{m}$ ). These DFB TDLs typically produce 5 to $25 \mathrm{~mW}$ of power with a laser linewidth $<5 \mathrm{MHz}$, leading to negligible instrument broadening. In addition, DFB TDLs are frequently available in fiber-pigtail packages further facilitating their integration into LAS sensors suitable for the field. In a DFB TDL, a p-n junction acts as a lasing medium and the bandgap (i.e., the energy gap between the conduction and valence bands) of the semiconductor sets the range of wavelengths emitted. A diffraction grating (located near the p-n junction) is used to effectively filter the emitted light and reflect a single wavelength back into the gain medium for lasing. By changing the 
1530 temperature of the laser (directly or via current tuning i.e., ohmic heating) the pitch of the grating is adjusted, and the emitting wavelength is tuned.

In practice, these lasers are typically operated by commercial temperature and current controllers with wavelength tuning achieved via external injectioncurrent modulation. Depending on the manufacturer and type of semiconductor used, the wavelength of DFB TDLs can typically be injection-current tuned from 1 to $10 \mathrm{~cm}^{-1}$ during DC operation. The tuning range typically decreases to around 0.01 to $0.1 \mathrm{~cm}^{-1}$ at modulation frequencies on the order of $100 \mathrm{kHz}$ $[40,155]$. This tunability enables the measurement of several adjacent absorption lines at modest rates (order of $1 \mathrm{kHz}$ ) [205] and portions thereof at high-frequencies

1545 (order of $100 \mathrm{kHz}$ ) as done in WMS [40, 166, 155].

\subsubsection{Vertical Cavity Surface Emitting Lasers (VC- SELs)}

VCSELs are a type of diode laser where light is emitted perpendicular to the semiconductor wafer (in comparison to edge-emitting lasers that emit radiation pase allel to the surface). The resonator is formed by two Bragg mirrors with an active region spanning only a few $\mu \mathrm{m}$ in-between. In comparison to edge-emitting DFB lasers, VCSELs produce much lower optical power (order of $1 \mathrm{~mW}$ ), however, can exhibit superior waves length tunability (near $10 \mathrm{~cm}^{-1}$ at modulation frequencies near $1 \mathrm{MHz}$ ). The latter of which has been exploited to acquire increased spectral information, for example, for resolving line-of-sight temperature dis-

1560 tributions [176] and collision-broadened lines in higippo pressure gases [33, 165]. At present, VCSELs are available from 750 to $1000 \mathrm{~nm}$ and near 1300, 1550 and $2000 \mathrm{~nm}$ and have been used to measure $\mathrm{O}_{2}[33,176]$, $\mathrm{H}_{2} \mathrm{O}$ [165], $\mathrm{CO}$ and $\mathrm{CO}_{2}$ [206], and $\mathrm{CO}$ [207], respectively, to name only a few examples. In addition, recently a micro-electro-mechanical (MEMS) VCSEL capable of scanning from 1330 to $1365 \mathrm{~nm}$ at $100 \mathrm{kHz}$ became commercially available and has been used for hyperspectral measurements of $\mathrm{H}_{2} \mathrm{O}$ and temperature in combustion flows [154].

\subsubsection{Fourier-Domain Mode Locked (FDML) Lasers}

FDML lasers [208, 209] are capable of providing broad spectral coverage (up to $100 \mathrm{~nm}$ ) at high repetition rates (order of $100 \mathrm{kHz}$ ) with a moderate linewidth

$1575(\approx 3 \mathrm{GHz})$. A schematic illustrating the operating pribl-5 ciples of FDML lasers is shown in Fig. 13 (taken from [210]). These lasers consist of three primary components: a fiber-coupled semiconductor optical amplifier (SOA), a ring-fiber-based resonator, and a Fabry-Perot

1580 tunable filter (FP-TF). Upon startup, broadband anbe plified spontaneous emission (ASE) leaving the SOA travels to the sinusoidally modulated FP-TF which

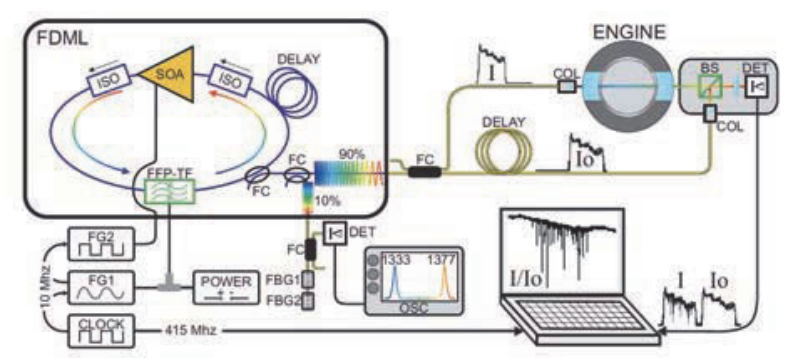

Figure 13: Schematic for hyperspectral absorption sensing using FDML lasers. Reprinted from [210] with permission from The University of Wisconsin.

passes a time-varying wavelength, thereby effectively sweeping the wavelength exiting the FP-TF. This filtered light (dispersed in time) then passes to the SOA for amplification via stimulated emission and the process repeats. By modulating the FP-TF with a drive period corresponding to the round-trip time of light in the cavity, each wavelength passed by the filter is passed on each subsequent roundtrip ensuring amplification and refinement of the wavelength sweep. The sweep rate can be varied by altering the length of the fiber-ring cavity [164].

FDML lasers have been deployed in a variety of combustion flows for hyperspectral ( $\approx 10$ to $100 \mathrm{~nm}$ ) measurements of $\mathrm{H}_{2} \mathrm{O}$ (and gas properties thereof) near 1.3 to $1.4 \mu \mathrm{m}[36,37,38,189,164]$. Their high sweep-rates makes them well suited for studying transient combustion flows, however, this combined with their broad spectral coverage mandates the use of extremely fast detection electronics (order of $100 \mathrm{MHz}-1 \mathrm{GHz}$ bandwidth) in order to resolve the fast wavelength scanning.

\subsubsection{Dispersed Supercontinuum Lasers}

Like FDML lasers, dispersed supercontinuum lasers (DSLs) can provide high-repetition rate, broadband measurements of absorption spectra with a moderate laser linewidth $(\approx 3 \mathrm{GHz})[211,212,213,214,215,216$, $217,128,218]$. To name a few examples, DSLs have been used to measure acetylene [211, 212, 213], and $\mathrm{H}_{2} \mathrm{O}$ and $\mathrm{CO}_{2}$ in gas cells [212], $\mathrm{CH}_{4}$ in atmospheric pressure jets [215] and flames [128, 218], and $\mathrm{H}_{2} \mathrm{O}$ in an HCCI engine [214].

These sources use a dispersing optical fiber (or other non-linear optical component) to separate the wavelengths contained within a broadband ps- or fs-pulse in time. The net result is that faster-travelling wavelengths reach the detector first, effectively producing a swept wavelength source and enabling the measurement of transmission spectra, and ultimately absorption spectra, in the time-domain. These concepts are illustrated in Fig. 14. Until recently, most previous studies using DSL were focused on fundamental 

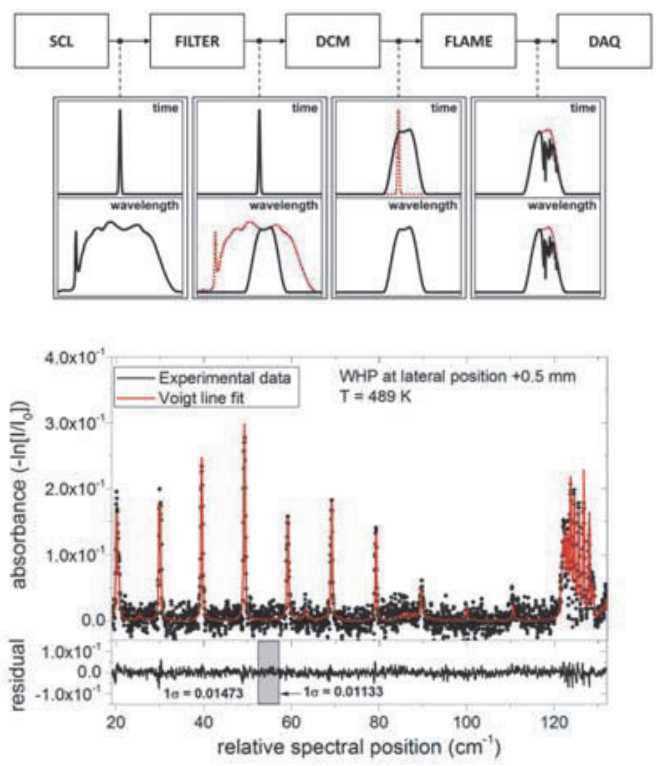

Figure 14: Schematic illustrating the principles of a supercore tinuum laser and example of $\mathrm{CH}_{4}$ spectra measured using a supercontinuum laser. Figures are reprinted from [128] with permission from The Optical Society of America.

studies with long and/or multi-pass path lengths (> $>$ FO $\mathrm{cm}$ ) and lacked use and validation in combustion gases [211, 212, 213, 215, 216, 217]. Blume and Wagner [128] recently reported single-pass measurements (spanning $4.1 \mathrm{~cm}$ ) of $\mathrm{CH}_{4}$ mole fraction in a well-characterized non-premixed, laminar $\mathrm{CH}_{4}$-air flame. The DSC source enabled measurement across $110 \mathrm{~cm}^{-1}$ of the $\mathrm{CH}_{4} \mathrm{ab}_{1680}$ sorption band near $1650 \mathrm{~nm}$ with a resolution of 0.152 $\mathrm{cm}^{-1}$ at a repetition rate of $2 \mathrm{MHz}$. A spectral-fitting routine was employed to calculate absolute mole fractions and measurements were able to resolve $3 \% \mathrm{CH}_{4}$ by volume (1210 ppm-m) after averaging 9760 pulses for a near $200 \mathrm{~Hz}$ measurement rate. The results were also shown to agree well with previous measurements acquired in the burner indicating that DSLs have the potential for accurate measurements of combustion species in flames.

\subsubsection{Near-Infrared Optical Fibers}

Silica-based fibers and fiber components have been developed by large investments from the telecommunications industry and, as a result, fiber optics (e.g95 combiners/splitters, rings) are widely available from 16451.25 to $2 \mu \mathrm{m}$. Now, single-mode and polarizationmaintaining fibers with low bend-losses are available well beyond $2 \mu \mathrm{m}$ (e.g., NuFern). These fiber components enable the lasers, detectors and automatic dater processing electronics to be remotely located away from temperatures and, potentially, large acoustic and electrical noise.

\subsection{Mid-Infrared Hardware}

Advances in mid-infrared $(\approx 2.5$ to $12 \mu \mathrm{m})$ optical equipment over the past 15 years has provided several new capabilities for LAS sensing in combustion environments. Most significantly, the fundamental vibrational frequencies of most combustion species are in this domain, and typically provide several orders of magnitude greater absorption relative to the overtone and combination bands in the near-infrared. This allows for (a) more sensitive detection and/or (b) detection at shorter pathlengths. Increasingly convenient access to the mid-infrared has enabled new applications of LAS in short path-length combustion systems that were simply not feasible in the near-infrared. For example, the high-fidelity measurements of $\mathrm{CO}$ in scramjet [219] and detonation combustors [220] were made possible through the use of quantum-cascade lasers enabling access to $10^{4}$ times greater absorption strength compared to that accessible via telecommunication-grade lasers. More of these new applications are highlighted in the latter part of this paper. Here we offer brief descriptions of key hardware for laser absorption in the mid-IR, with a focus on recent advancements.

\subsubsection{Quantum Cascade Lasers (QCLs)}

QCLs are semiconductor lasers that can emit in the mid-infrared to far-infrared $(\approx 3.5$ to $100 \mu \mathrm{m})$ with similar tuning capability and usability to roomtemperature diode lasers. Unlike diode lasers the emission wavelength is not purely a material property (i.e. band gap), but rather the result of an engineered superlattice, a layered composite of semiconducting materials that introduces a variable electric potential across the length of the system. Electron transitions and photon emission occur and cascade (via tunneling) between the subbands of each layer. The respective energy levels between each layer and emission wavelength depend on the respective layer thicknesses and can be designed to cover a broad spectral region with a given material set. The first demonstration of the quantum cascade laser occurred at Bell Laboratories in 1994 [221]. Rapid improvement in manufacturing and lasing properties (i.e., tuning range, efficiency, power) has followed [222, 223, 224]. Similar to diode lasers, wavelength tuning of a QCL can be achieved with a distributed feedback (DFB) or external cavity architecture, with the latter offering greater range $\left(\approx 100 \mathrm{~cm}^{-1}\right)$ at the expense of slower tuning and reduced scan-to-scan repeatability. Both tuning architectures can provide narrow linewidth emission $(<5 \mathrm{MHz})$, suitable for the LAS techniques discussed previously. QCLs are now 
available for a wide range of wavelengths from multiple vendors (e.g., Alpes, Adtech, Daylight Solutions, Hamamatsu, Pranalytica).

\subsubsection{Interband Cascade Lasers (ICLs)}

ICLs leverage interband transitions and electron-hole recombination similar to a diode, but also use a layered heterogeneous bandstructure to achieve cascading of 1710 photon emission similar to a QCL. As such, ICLs may be viewed as a hybrid of conventional diode lasers and quantum cascade lasers. The use of interband transitions (as opposed to intersubband transitions) allows for lower electrical input powers compared to QCLs, but also yields a more narrow range of wavelengths over which lasing can be achieved at room temperature $(\approx$ 2.9 to $5.7 \mu \mathrm{m})$ [225]. Invented around the same time as the QCL was demonstrated [226], the first continuous wave ICL was demonstrated in 2008 [227]. Subse-

1720 quent advancements in tuning response and efficiency $[228,229]$ have enabled commercially available, compact, narrowband DFB lasers that fill a gap $(\approx 3$ to $4 \mu \mathrm{m})$ in the mid-wave infrared between conventional room-temperature diodes and quantum cascade lasers.

1725 This region is especially important to combustion due to the very strong $\mathrm{C}-\mathrm{H}$ stretch vibrational mode common to all hydrocarbons near $3.4 \mu \mathrm{m}$.

\subsubsection{Mid-IR Optical Fibers}

Since conventional silica-based fibers do not transmit light beyond $\approx 2.3 \mu \mathrm{m}$, alternatives are required for the mid-IR. Fiber materials for the mid-infrared are more expensive, generally less robust mechanically, and have higher attenuation, thus limiting the length that is acceptable for meaningful transmission levels. portant than transmission loss for the relatively short fiber length $(<100 \mathrm{~m})$ needed to remotely locate combustion sensors. With increasing demand for mid-IR fibers, a number of materials have matured commer1740 cially in the last fifteen years or so $[230,231]$. A present, several types of mid-infrared fibers are available for use in harsh environments. Figure 15 highlights some of these fiber materials and their respective transmission spectra in the infrared. Two of the more attractive fiber types are fluoride glasses (ZBLAN arre $\mathrm{InF}_{3}$ ) and hollow-core waveguides. Both fiber types are mechanically more robust than chalcogenide, which is known to be brittle [232] and together offer transmission across a broad wavelength range from $\approx 2$ to 12

$1750 \mu \mathrm{m}$. The fluoride glass fibers offer lower attenuation than the hollow-core waveguides in the 2 to $5 \mu \mathrm{m}$ wavelength domain and are available in greater lengths $(>$ $5 \mathrm{~m})$. The fluoride glass fibers are also available as single-mode and were successfully utilized for multiple

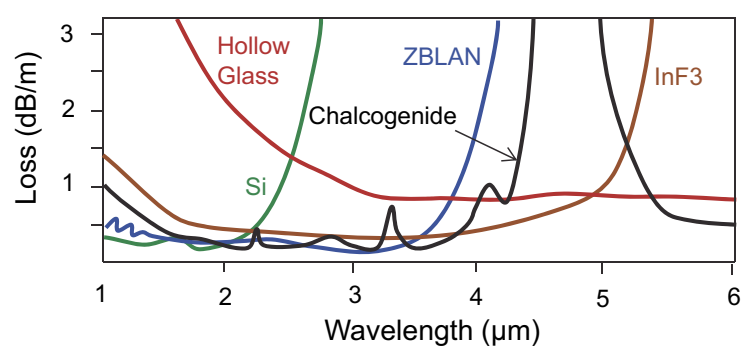

Figure 15: Transmission loss of various mid-IR optical fibers as a function of wavelength. Figure reprinted from [233] with permission from Stanford University.

tion 12). The hollow-core waveguides offer transmission over a broader wavelength range compared to the fluoride glasses and also typically come in larger diameters for ease of coupling which is especially needed for non-Gaussian beams. Additionally, because there is no solid core to the fiber, there is less risk of spurious back reflections that often occur during fiber coupling and lead to wavelength and intensity jitter. The hollowcore fibers do tend to induce substantial light attenuation when bent and may require purging to avoid absorption due to ambient $\mathrm{CO}_{2}$ and $\mathrm{H}_{2} \mathrm{O}$.

\subsubsection{Mid-IR Optical Materials}

Table 3 lists a number of common optical materials (frequently used as windows) that transmit in the infrared. Quartz, or fused silica $\left(\mathrm{SiO}_{2}\right)$, is the most common and inexpensive optical material, but it only transmits up to $\approx 3.5 \mu \mathrm{m}$, thus it is not suitable for sensing in most of the mid-infrared. Sapphire $\left(\mathrm{Al}_{2} \mathrm{O}_{3}\right)$ is an excellent material for harsh environments due to its superior strength and high melting point, which makes it suitable for most high-pressure combustion applications. Sapphire also has relatively high thermal conductivity and low thermal expansion, making the material highly resistant to thermal shock. For many cases, sapphire is the ideal material selection for optical ports/windows in high-temperature gas flows. However, sapphire transmission degrades around $5 \mu \mathrm{m}$ and the transmission cutoff has a strong temperature dependence. This should be considered when designing an LAS sensor for $\mathrm{CO}_{2}(\approx 4.3 \mu \mathrm{m}), \mathrm{CO}(\approx 4.8 \mu \mathrm{m})$, or NO $(\approx 5.2 \mu \mathrm{m})$ at high temperatures. An empirical model of the temperature-dependent transmission of sapphire was developed by Thomas et al. [234], and results are shown in Figure 16 for a temperature range of 300 to $2100 \mathrm{~K}$ with a window thickness of $1 \mathrm{~cm}$. If probing a wavelength in the $\approx 3.5$ to $6 \mu \mathrm{m}$ domain, it is important to understand that time-varying transmission may occur (e.g., during thermal transients) if using the diagnostic in a high-temperature system.

Transmission cutoffs aside, quartz and sapphire are 
Table 3: Properties of mid-infrared optical materials.

\begin{tabular}{cccccc}
\hline Material & $\lambda_{\text {low }}[\mu \mathrm{m}]$ & $\lambda_{\text {high }}[\mu \mathrm{m}]$ & $T_{\text {melt }}[\mathrm{K}]$ & $n_{\text {refractive }}$ & $E_{\text {rupture }}[\mathrm{ksi}]$ \\
\hline $\mathrm{SiO}_{2}$ & 0.16 & 3.5 & 1980 & 1.5 & 7 \\
$\mathrm{Al}_{2} \mathrm{O}_{3}$ & 0.17 & 5 & 2300 & 1.75 & 65 \\
$\mathrm{BaF}_{2}$ & 0.15 & 12 & 1620 & 1.45 & 3.9 \\
$\mathrm{CaF}_{2}$ & 0.15 & 8 & 1700 & 1.4 & 5.3 \\
$\mathrm{MgF}_{2}$ & 0.13 & 7 & 1520 & 1.35 & 7.2 \\
$\mathrm{ZnSe}$ & 0.55 & 20 & 1800 & 2.4 & 8 \\
$\mathrm{ZnS}$ & 0.38 & 14 & 1450 & 2.5 & 8.7 \\
$\mathrm{Ge}$ & 1.8 & 21 & 1200 & 4 & 7 \\
$\mathrm{Si}$ & 1.2 & 10 & 1690 & 3.5 & 18.1 \\
\hline
\end{tabular}

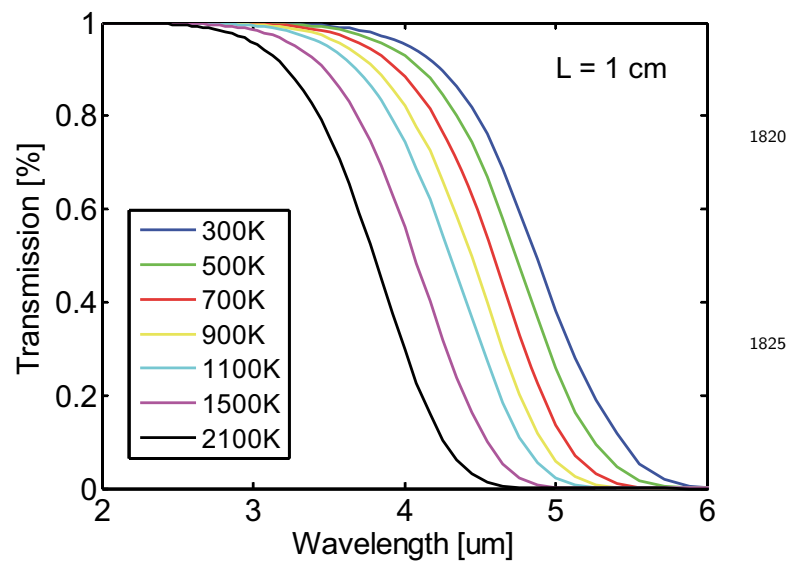

Figure 16: Transmission through a $1 \mathrm{~cm}$ thick sample of sap3o phire as a function of wavelength for various temperatures. Results were calculated using the model presented in [234]. Figure reprinted from [233] with permission from Stanford University.

the best infrared materials for spectroscopic windows on harsh combustion systems and should be used whæm feasible. Other classes of mid-infrared optical materials are less robust than quartz or sapphire but may crystals including $\mathrm{BaF}_{2}, \mathrm{CaF}_{2}$, and $\mathrm{MgF}_{2}$ have broad transmission from the ultra-violet up to $12 \mu \mathrm{m}$ (in that case of $\left.\mathrm{BaF}_{2}\right)$ and low indices of refraction $\left(n_{\text {refractive }}\right)$ which helps mitigate back reflections and etalons, but they are somewhat weak and highly susceptible to thermal shock due to their large thermal expansion coefficients. Furthermore, when heated above $800 \mathrm{~K}$ tha fluoride crystals react with water vapor. Zinc Selenide $(\mathrm{ZnSe})$ and Zinc Sulfide $(\mathrm{ZnS})$ offer even broader transmission into the far-infrared (up to $20 \mu \mathrm{m}$ ). They are a bit stronger than the fluoride crystals, and offer greater resistance to thermal shock, but are relatively britso tle. Perhaps most problematic for high-temperature applications, these zinc materials oxidize above 500 $\mathrm{K}$. The surface reaction issues with both the fluoride and zinc optical materials may be resolved with high- temperature coatings. Germanium and Silicon are also good infrared optical materials at room temperature, but these materials rapidly become opaque as temperature is elevated [235]. It should be noted that for shock tube applications, the windows are only exposed to a high temperature gas for a very short time period (order of ms), thus many of these temperature-dependent issues are less important, since minimal heat transfer occurs during that time.

\subsection{Emerging Sources}

This section will discuss several novel light sources and/or techniques that have recently been developed and demonstrated in IR-LAS sensors. These methods have been shown to offer improvements ranging from measurement rate to spectral bandwidth and measurement fidelity.

\subsubsection{Chirped QCLAS}

Chrystie et al. [236] recently demonstrated the use of a chirped-QCL for measurements of $\mathrm{H}_{2} \mathrm{O}$ near 7.62 $\mu \mathrm{m}$ at rates as high as $3.125 \mathrm{MHz}$ and temperature measurements at up to $250 \mathrm{kHz}$. The authors demonstrated this technique with measurements acquired in a static cell at known conditions and behind reflected shock waves in a reacting gas, thereby demonstrating the utility of this method for studying highly transient combustion processes (e.g., chemical kinetics). The ability to acquire calibration-free species and temperature measurements at $\mathrm{MHz}$ (or near) rates that employ wavelength tuning for increased robustness represents a significant advancement. This achievement has the potential for offering improved understanding of a variety of highly-transient combustion processes.

This technique operates by pulsing a QCL's current with a high-frequency, long-duration squarewave which, within the pulse, rapidly increases the temperature of the laser causing its wavelength to vary (i.e., chirp). In LAS applications, the pulse duration and amplitude are specified such that the laser's wavelength 
a)

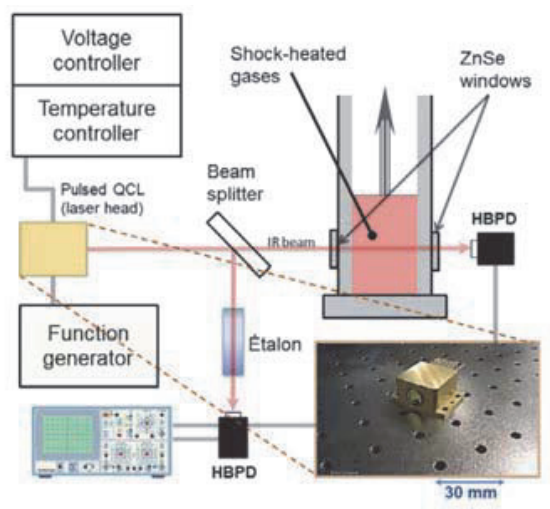

b)

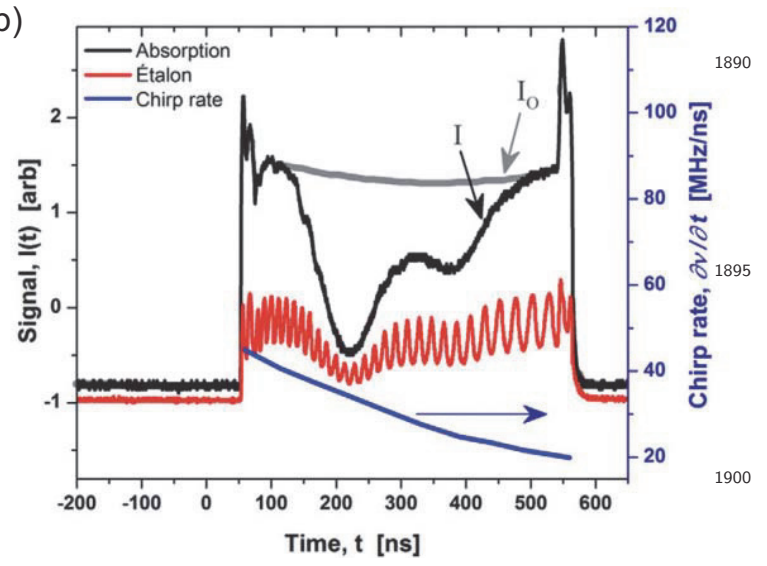

Figure 17: Schematic of experimental setup for chirped QCLAS (a) and example of raw transmission data for a single pulse chirped across $\mathrm{H}_{2} \mathrm{O}$ absorption transitions near $7.6 \mu \mathrm{m}$ ( $\mathrm{b}_{965}$ Reprinted from [236] with permission from The Combustion Institute.

chirps (i.e., tunes) across an absorption transition analogous to scanned-wavelength direct-absorption experio ments. In this method, a primary concern is the chirpinduced broadening of the laser linewidth. The greater the chirp rate, the greater the instantaneous linewidth [236] exhibited chirp rates from 20 to $60 \mathrm{MHz} / \mathrm{ns}$ yield[236 exhibited chinp rates from 20 to $60 \mathrm{MHz} / \mathrm{ns}$ yieldultimately the limiting factor preventing faster thermometry measurements (since a greater chirp rate is needed to scan across two transitions). These concepts are illustrated in Figure 17.

\subsubsection{Frequency Comb Spectrometers}

Recently, Rieker et al. [237] used a dual-comb spectrometer (DCS) to measure gas temperature, $\mathrm{H}_{2} \mathrm{O}$, $1870 \mathrm{HDO}, \mathrm{CO}_{2},{ }^{13} \mathrm{CO}_{2}$, and $\mathrm{CH}_{4}$ simultaneously across a 2 $\mathrm{km}$ open path through the turbulent atmosphere. Tlare DCS spanned 1600 to $1670 \mathrm{~nm}$ with 80,000 comb teeth and a tooth spacing of $100 \mathrm{MHz}$. The authors reported absorbance noise levels below $5 \times 10^{-4}$ enabling one-
$\mathrm{CH}_{4}$ after 5 minutes of signal averaging.

The operating principles of a DCS are shown in Fig. 18 (taken from [237]). In a DCS, two frequency combs with teeth at optical frequencies (i.e., in the $\mathrm{THz}$ regime) are locked together with a fixed offset $\left(\Delta f_{r}\right)$ in their repetition rate $\left(f_{r}\right)$. These combs are combined together to create a $r f$-comb (via heterodyning) where each tooth (in the $\mathrm{MHz}$ regime) exhibits a one-to-one relationship with its corresponding pair of $\mathrm{THz}$ teeth that are attenuated according to Beer's Law and the absorbance spectra of each chemical species in the test gas. The measured detector signal therefore yields an interferogram which can be processed to yield a measured transmission and absorption spectrum.

DCSs offer a number of unique capabilities including broad spectral coverage (e.g., 5990 to $6260 \mathrm{~cm}^{-1}$ in [237]), ultra-narrow linewidth (order $1 \mathrm{~Hz}$ per comb tooth), and precisely known absolute wavelength (frequency of each tooth known to better than $1 \mathrm{kHz}$ ). These attributes make DCSs ideal tools for high-fidelity measurements of molecular spectra and are in stark contrast to other hyperspectral sources (e.g., FDML, Supercontinuum) that exhibit comparatively broad linewidths on the order of $0.1 \mathrm{~cm}^{-1}(2.99 \mathrm{GHz})$ or lack wavelength stability (e.g., external cavity quantumcascade lasers). These benefits, however, currently come with the requirement to average many pulses which reduces the ultimate measurement rate. Regardless, it is clear that DCS-based sensors offer great potential in expanding upon the measurement capabilities offered by more established IR-LAS technologies.

During the compilation of this manuscript, Schroeder et al. [238] presented the first use of a DCS laser-absorption sensor in an industrial environment. The authors presented measurements of temperature, $\mathrm{H}_{2} \mathrm{O}$, and $\mathrm{CO}_{2}$ at 1 to $10 \mathrm{~Hz}$ in a 16 MW gas-turbine exhaust, thereby demonstrating the potential of this technology to be applied to a wide range of practical combustion systems.

\subsection{Compact Sensors}

The need to characterize combustion systems with limited optical access has driven the development of numerous compact LAS sensors $[239,240,42,241$, $242,158,164,243,183,244,245,246,154,247]$ and the majority of these sensors have leveraged robust telecommunication-grade optical hardware. Specifically, Wehe et al. [239, 240] embedded a diode-laserbased sensor into a probe for gas temperature, velocity, and $\mathrm{H}_{2} \mathrm{O}$ in hypervelocity flows, Schultz et al. [243] and Strand and Hanson [244] developed fibercoupled sensor packages for model scramjets housed in vacuum chambers, Ebert et al. [247] and Whitney et al. [158] developed compact arrangements for providing line-of-sight access in IC engines, and Caswell 

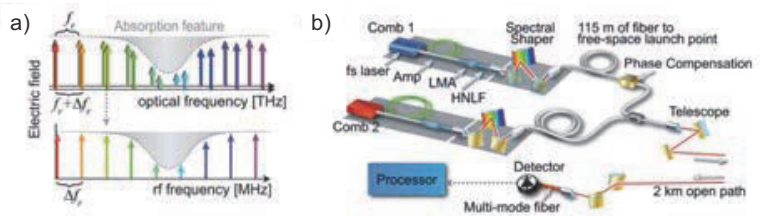

c)

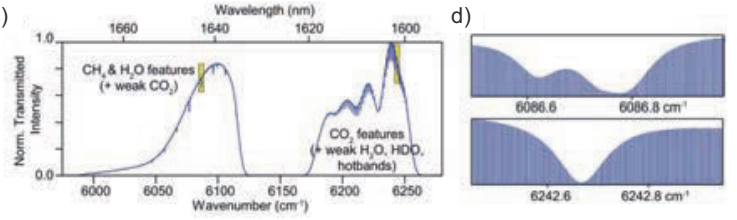

Figure 18: Frequency spectrum of DCS interrogating an absorption transition (a), schematic of experimental setup (b) and example transmission spectra (c) and (d). Figures adapted from [237].

et al. [164] developed a fiber-optic package providing counter-propagating beams for facilitating velocimetry sensing. While the aforementioned examples are certainly compact compared to other laser-diagnostic techniques (e.g., PLIF, CARS), this section will highlight only the most compact sensors, specifically, singleended LAS sensors.

\subsubsection{Probe-Based Single-Ended Sensors}

Several researchers have developed single-ended probes for studying IC-engines via IR-LAS [248, 249, 42, 250, 241, 242]. Hall and Koenig [248] were the first to implement IR fibers into a spark-plug probe (similar to that shown in Figure 19) for delivering and receiving IR light. The authors used a broadband IR source to monitor the absorption of fuel from 3.3 to $3.5 \mu \mathrm{m}$, thereby enabling monitoring of the fuel-air ratio near the spark plug. A similar configuration and diagnos tic technique was more recently used by Cundy et al. [250]. Tomita et al. [249] used a similar probe design, but used two sapphire rods cleaved at $45^{\circ}$ to direct light and of the engine and used a HeNe near $3.39 \mu \mathrm{m}$ to monitor fuel absorption. More recently, Rieker et [42] integrated a fiber-coupled WMS- $2 f / 1 f$-based TDLAS sensor into a spark plug and demonstrated crankangle resolved measurements of temperature and $\mathrm{H}_{2} \mathrm{O}$ concentration in motored and fired tests. The sensor used a SMF to pitch laser light near $1.4 \mu \mathrm{m}$ across a $6 \mathrm{~mm}$ gap and a small mirror was used to reflect light back towards a multi-mode catch fiber (see Fig. 19). Jeffries et al. [241] later used a similar probe line. The authors used a fiber-coupled DFG techniqid\& to generate two colors near 2951 and $2970 \mathrm{~cm}^{-1}$ for exploiting the strong $\mathrm{C}-\mathrm{H}$ stretch absorption band for high-sensitivity measurements of gas temperature and gasoline concentration. The injection current of each

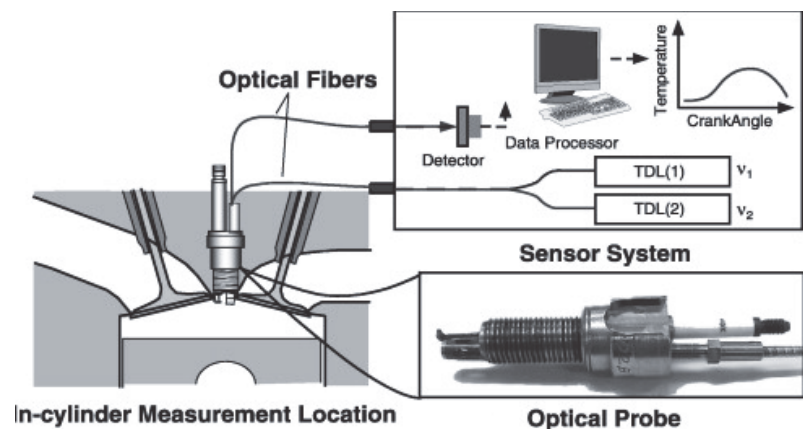

Figure 19: Schematic of fiber-coupled TDLAS temperature and $\mathrm{H}_{2} \mathrm{O}$ sensor embedded into a spark plug. Reprinted from [42] with permission from The Combustion Institute.

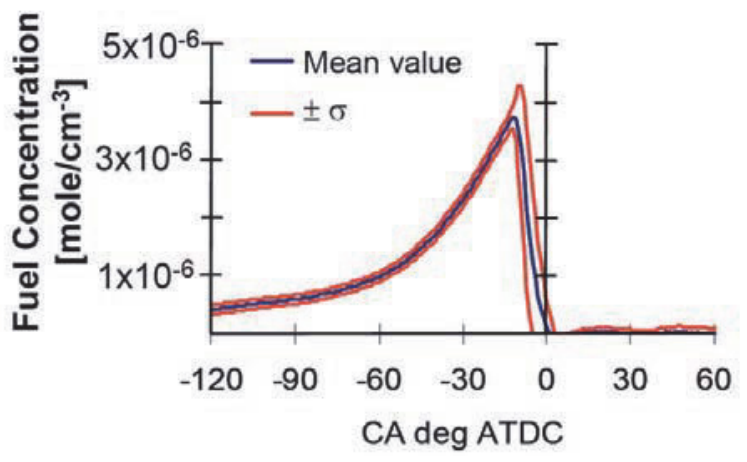

Figure 20: Time-resolved measurements of gasoline concentration acquired near spark plug using WMS-1 $f$ sensor during fired operation. Reprinted from [241] with permission from SAE.

pump laser was modulated at a unique frequency to enable frequency-multiplexing and demultiplexing of each color with a sensor bandwidth of $10 \mathrm{kHz}$. The WMS$1 f$ signal of each laser was then extracted via postprocessing using digital lock-in amplifiers and the twocolor ratio of WMS- $1 f$ signals was used to calculate temperature analogous to as done in fixed-wavelength direct-absorption techniques. Details regarding the development of this sensor can be found in [168] and quantitative measurements of gasoline were enabled via a spectroscopic model developed by Klingbeil et al. [44]. Fig. 20 illustrates time-resolved measurements of gasoline concentration acquired at pressures up to 40 atm during fired operation. Measurements of air-fuel ratio acquired during operation agreed well with the air-fuel ratios inferred from analysis of independently acquired exhaust emissions measurements. In addition, Rein and Sanders [242] also used a similar probe in an IC-engine, but used a fiber-coupled FTIR spectrometer to measure $\mathrm{H}_{2} \mathrm{O}, \mathrm{CO}_{2}$ and temperature. While useful, the primary disadvantage of all the aforementioned designs is that probes can perturb the local flow field and heat transfer to the probe surfaces can influence the local gas temperature and perturb the local chemistry. 
a)

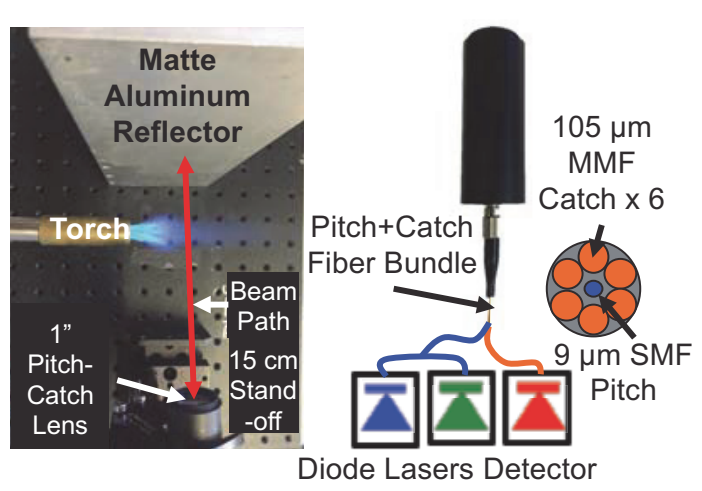

b)

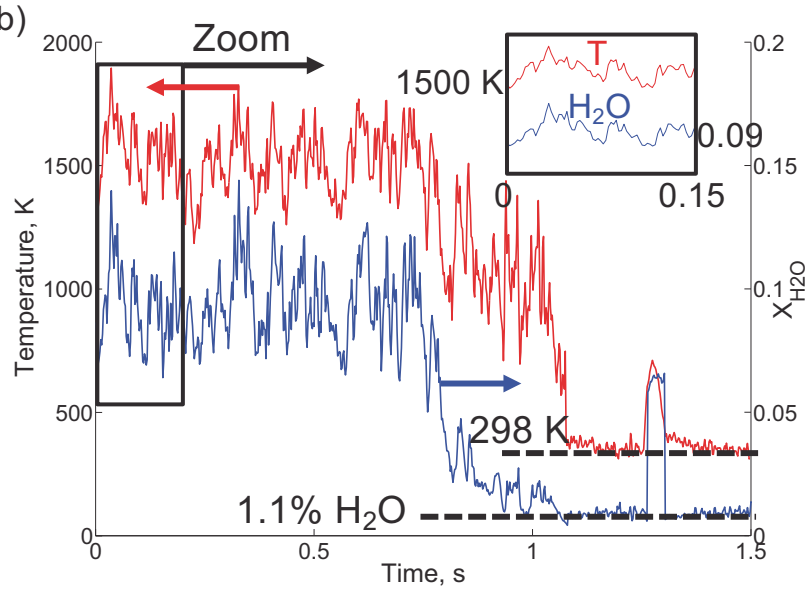

Figure 21: Schematic of a single-ended LAS sensor using a fiber-bundle (a) for stand-off measurements of gas temperature, pressure, and $\mathrm{H}_{2} \mathrm{O}$ near $1.4 \mu \mathrm{m}$ (b). Figures adapted from [246].

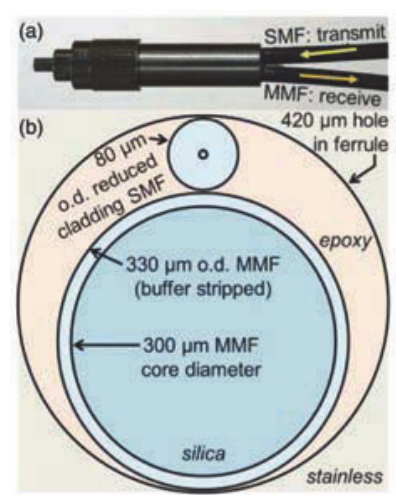

Figure 22: Schematic of dual fiber-optic used to acquire singleended measurements of gas temperature and $\mathrm{H}_{2} \mathrm{O}$ in a rotatingdetonation combustor in [154]. Figure reprinted from [164] with permission from The Optical Society of America.

\subsubsection{Single-Ended Sensors Using Native Surfaces}

Due to the intrusive nature of probes, several researchers have recently developed single-ended LAS sensors relying on backscattering off native surfaces (e.g., a combustor wall) $[245,246,154]$. Wang and Sanders [245] recently demonstrated a single-ended near-IR LAS sensor for monitoring $\mathrm{H}_{2} \mathrm{O}$ near 1.4 with scanned-WMS- $2 f / 1 f$. They showed that WMS$2 f / 1 f$ with $f=100 \mathrm{kHz}$ was able to reject speckle noise and demonstrated accurate measurements off a age could be deployed in an engine/combustor with moving parts. Goldenstein et al. [246] developos a single-ended, fiber-coupled near-IR diode-laser sensor for stand-off measurements of gas temperature, pressure, and composition using scanned-WMS- $2 f / 1 f$. This sensor employed a fiber-bundle with 6 multi-mode catch fibers surrounding a single single-mode pitch fiber housed in a $25.4 \mathrm{~mm}$ diameter lens tube. This configuration was shown to provide collection efficiencies (fraction of incident photons collected) from 1 to $10^{4}$ parts-per-million at stand-off distances from $10 \mathrm{~m}$ to $10 \mathrm{~cm}$, respectively. The sensor was demonstrated with measurements of temperature, pressure, and $\mathrm{H}_{2} \mathrm{O}$ in a flame at a stand-off distance of $15 \mathrm{~cm}$ and $\mathrm{CH}_{4}$ at a stand-off distance of $10 \mathrm{~m}$. Diode lasers near 1.4 $\mu \mathrm{m}$ and $1.65 \mu \mathrm{m}$ were used to interrogate $\mathrm{H}_{2} \mathrm{O}$ and $\mathrm{CH}_{4}$ transitions, respectively. A schematic showing the sensor package is shown in Fig. 21. Most recently, Rein et al. [154] deployed a single-ended, fiber-coupled MEMS VCSEL sensor operating near $1.4 \mu \mathrm{m}$ for measurements of temperature and $\mathrm{H}_{2} \mathrm{O}$ at $100 \mathrm{kHz}$ in a rotating-detonation combustor (RDC). This sensor utilized a single-mode pitch fiber and multi-mode catch fiber housed side-by-side in the fiber bundle developed by Caswell et al. [164] (see Figure 22) to transmit and receive laser light reflected off the RDC centerbody. A single lens was used to collimate the incident light and collect the backscattered light after traversing $1.52 \mathrm{~cm}$ (round trip) through the test gas.

\section{Sensors for Fundamental Combustion Chem- istry Studies}

Here we begin our review of recent applications of IRLAS for studying combustion gases with an overview of sensors used for investigating combustion chemistry. These systems include various flame apparatuses as well as shock tubes, where the primary goal is the determination of fundamental rate constants or insights about reaction pathways that describe the kinetic behavior of fuels or fuel byproducts. 


\subsection{Shock Tubes}

Studies of chemical kinetics in shock tubes have driven the development of a wide range of IR-LAS sensors. Shock tubes provide exceptionally uniform properties along the line-of-sight and are relatively quicent (compared to most combustion flows), providing ideal conditions for high-fidelity time-resolved LA $A^{12}$ measurements of species and gas properties. In reciprocity, species time histories, especially those with multiple time-scales, place internal constraints on comparameters such as ignition delay times, and have ledes to significant advances in the modeling of combustion chemistry [251]. As such, the application of infrared LAS in the context of shock tube kinetics has a rich history that has been discussed in other review papers, including the recent paper by Hanson and Davidsơfl [122]. Here we highlight only very recent works in order to identify emerging areas in IR LAS for combustion, which are often first developed and demonstrated on

2060 shock tubes, prior to utilization in other environments.

\subsubsection{Thermometry}

2115

In chemical-kinetics studies, accurate knowledge of temperature is critical for the determination of rate constants due to their exponential temperature depentubes, test temperature can be well-defined $(\approx 1 \%$ umpe certainty) by the measurement of incident shock velocity and utilization of the normal-shock relations. However, in experiments with transient temperature, only the initial value is well known by this method, and there are also cases where temperature measurements better than $1 \%$ uncertainty are desired as temperature may still be the largest uncertainty factor when propagated to a measured rate constant. Given the rel-

2075 ative importance of temperature, a number of efforts over the past ten years have been dedicated to proviobe ing a sensitive LAS-based thermometer for shock-tube studies. Several nascent combustion species (e.g., $\mathrm{H}_{2} \mathrm{O}$, $\mathrm{CO}, \mathrm{NO}$ and $\mathrm{CO}_{2}$ ) that are infrared active have been investigated for two-color thermometry, with reported uncertainties $(\approx 1 \%)$ that are competitive with the cons ventional approach while also providing time-resolved data for transients [252, 101, 253].

$\mathrm{H}_{2} \mathrm{O}$ and $\mathrm{CO}_{2}$ have received perhaps the most attention for shock tube thermometry, being relatively chemically inert and major combustion products thato can also be seeded into an initial reactant mixture. The mid-infrared spectra, and recent advances in laser technology in this domain, has enabled notable progress in the development of LAS sensors interrogating these species. Farooq et al. [98, 254] and Ren et al. [255] utilized the $\mathrm{CO}_{2}$ spectra near $2.7 \mu \mathrm{m}$ for temperature sensing behind reflected shock waves using two-color wavelength-modulation spectroscopy. Spearrin et al. $[99,256]$ later introduced a cross-band thermometry technique for $\mathrm{CO}_{2}$ that leverages the inverse temperature dependence of two lines from the $2.7 \mu \mathrm{m}$ and 4.2 $\mu \mathrm{m}$ vibrational bands, respectively, for enhanced sensitivity and a broader temperature range. Recent efforts for $\mathrm{H}_{2} \mathrm{O}$ thermometry include a pulsed-scanning, or intra-pulse chirp method, developed by Chrystie et al. [236] to resolve $\mathrm{H}_{2} \mathrm{O}$ lineshapes near $7.6 \mu \mathrm{m}$ as already discussed in Section 6.3. Campbell et al. [256] has further shown that scanned-WMS may be used for partially-resolving $\mathrm{H}_{2} \mathrm{O}$ lineshapes while still temporally-resolving temperature transients behind reflected shock waves. Though both species $\left(\mathrm{CO}_{2}\right.$ and $\mathrm{H}_{2} \mathrm{O}$ ) are very useful, $\mathrm{CO}_{2}$ does not readily adsorb to surfaces and has a much higher vapor pressure, making it easier to seed into a gas mixture.

\subsubsection{High-Pressure Studies and Sensor Development}

Species sensing at high pressures is another important capability for shock tube studies (as well as other combustion experiments) as most practical combustion devices operate at tens or hundreds of atmospheres, and there is a need for improving our understanding of kinetic behavior in this domain. LAS is inherently more challenging to implement at elevated pressures due to lineshape broadening and the associated blending of many spectral lines that complicate the interpretation of an absorption measurement, not to mention the practical challenges of increased beam steering and boundary layer effects. It can be quite challenging to validate absorption models (a prerequisite for quantitative LAS) at high-pressure and high-temperature conditions as creating a controlled, well-known environment at these extremes is difficult. Shock tubes serve an important role in this regard since temperature and pressure can be known quite well behind the reflected shockwave (albeit for short time) in a nonreactive experiment. Further, the impulsive nature of the experiment also lends to greater safety than static or steady-flow experiments since the extreme conditions behind the reflected shock typically only lasts a few milliseconds. Shock tubes have played a critically important role in the development of high-pressure infrared LAS diagnostics in recent years, in addition to facilitating kinetics studies. Here we identify a few notable works that extend measurement capabilities to pressures above $10 \mathrm{~atm}$.

The helium-neon gas laser near $3.39 \mu \mathrm{m}$ has been used for fixed-wavelength LAS measurements of numerous hydrocarbons for fuel pyrolysis and oxidation studies in shock tubes $[257,258]$. Similarly, the carbon dioxide gas laser $(\approx 10.6 \mu \mathrm{m})$ has been used for ethylene measurements at pressures higher than 50 atm $[258,259,260]$, with much of the development 

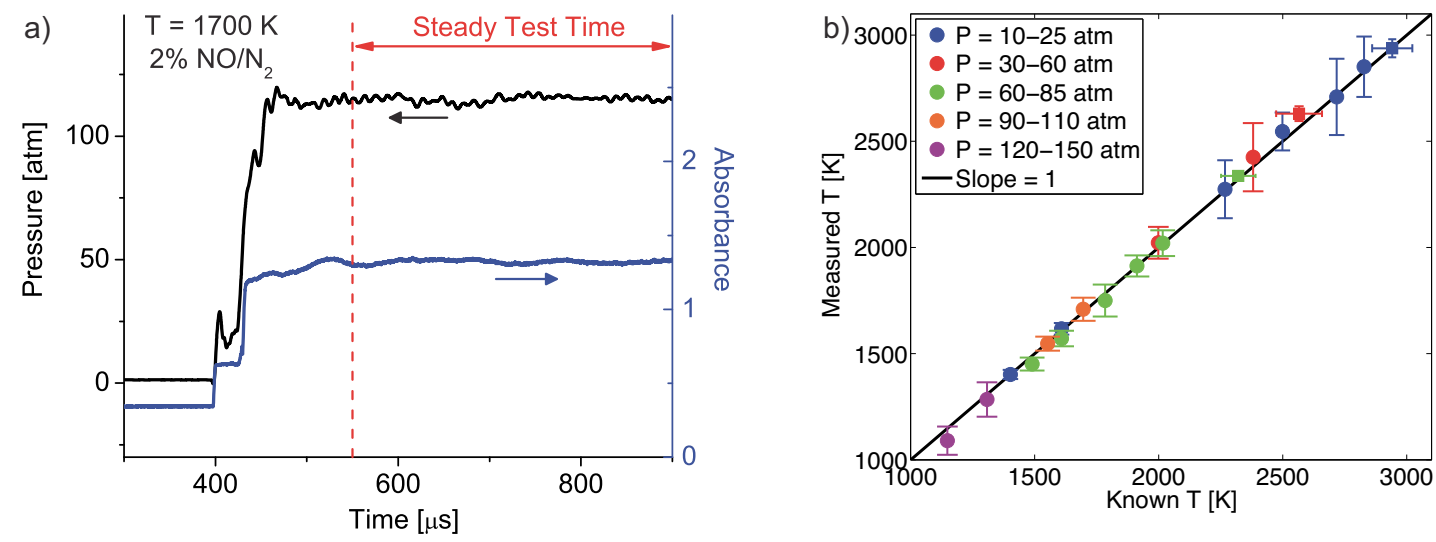

Figure 23: Measured time history of NO absorption behind a reflected shock wave (a) and summary of thermometry performance (b) for LAS thermometer based on equilibrium of nitric oxide at high pressures (15 to 150 atm) and temperatures. Reprinted from [111] with permission from IOP Publishing.

effort led by Pilla and Ren. Often, these two gas lasers are used simultaneously to observe larger hydro2150 carbons decomposing into smaller hydrocarbons (e.gsq ethylene). Semi-conductor diode and quantum-cascade lasers have enabled the detection of several other combustion species at elevated pressures. Farooq et al. [167] demonstrated WMS of $\mathrm{CO}_{2}$ near $2.7 \mu \mathrm{m}$ above 10 atm during heptane oxidation in a shock tube. Moss recently, Spearrin et al. [97, 102] and Goldenstein et al. [162, 155] used these tunable sources for acquiring measurements of $\mathrm{CO}_{2}, \mathrm{CO}, \mathrm{H}_{2} \mathrm{O}$, and temperature at pressures up to $\approx 50 \mathrm{~atm}$ in a shock tube, and later ap2160 plied these sensors to a pulsed-detonation combustrous [220]. For thermometry in high-enthalpy air, Spearrin et al. [111] validated and demonstrated a sensor based on NO detection at pressures up to $\approx 150 \mathrm{~atm}$ in a shock tube using a quantum-cascade laser near

$21655.2 \mu \mathrm{m}$ [111]. This sensor inferred the temperatures by comparing the measured concentration of $\mathrm{NO}$ to that given by temperature-dependent thermochemical equilibrium calculations; example results are shown in Figure 23.

\subsubsection{Fuel Intermediates}

At high temperatures, large hydrocarbon fuels breakdown into smaller intermediate species. Timehistories of fuel intermediates during combustion typically occur on at least two time-scales: formation aprob decomposition. Multiple time scales provide more rigid constraints for kinetic modeling which makes these species, similar to other intermediates like $\mathrm{OH}$ and
$\mathrm{CO}$, high-value targets in shock tube studies. Unfortunately, most hydrocarbons have been historically inaccessible in the infrared due to gaps in wavelength coverage for semiconductor lasers despite the strong fundamental vibrational bands such as $\mathrm{C}-\mathrm{H}$ stretch $(\approx 3$ $\mu \mathrm{m})$ and $\mathrm{C}-\mathrm{H}$ bend $(\approx 10-12 \mu \mathrm{m})$ that are shared by many important combustion species. Gas lasers such as the $\mathrm{HeNe}$ and $\mathrm{CO}_{2}$ lasers have enabled some species sensing in this domain, but the lack of continuous tuning capability and usual interference associated with a mixture of hydrocarbons has limited the expansion of these LAS methods beyond a few species. Fortunately, the emergence of interband and quantum cascade lasers over the past fifteen years has removed this constraint, providing near continuous wavelength coverage from the near to far infrared $(\approx 1-15 \mu \mathrm{m})$ via tunable, roomtemperature, coherent light sources. Correspondingly, this has led to many new sensors for fuel intermediates.

As mentioned previously, early work in the detection of fuel intermediates for shock tube kinetics studies focused on ethylene due to its importance and abundance in large alkane decomposition pathways and accessibility with a $\mathrm{CO}_{2}$ gas laser near $10.6 \mu \mathrm{m}[107,260]$. This was followed by efforts that utilized difference frequency generation (DFG) lasers to target methane [261] and formalydehyde [262] in the 3.4 to $3.6 \mu \mathrm{m}$ region. Improved methods for methane detection with regards to interference in combustion gases have more recently been developed by Sur et al. [106] using an interband cascade laser near $3.2 \mu \mathrm{m}$ and Sajid et al. $[105,263]$ using a quantum-cascade laser near $8 \mu \mathrm{m}$. 


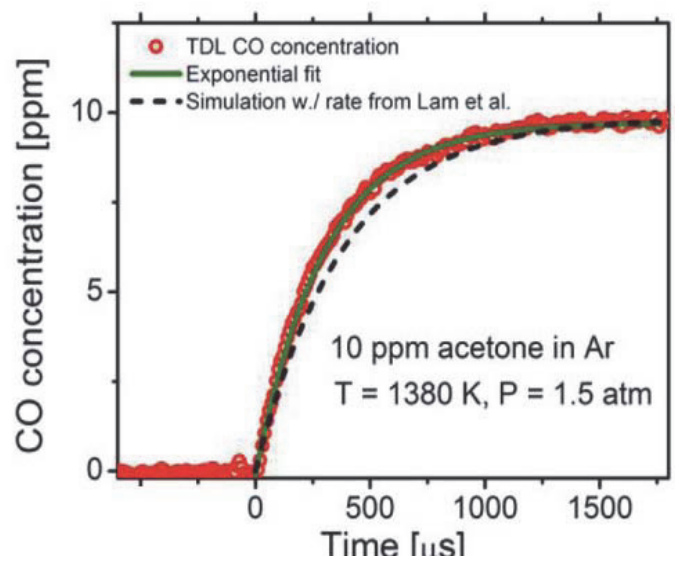

Figure 24: Formation of $\mathrm{CO}$ (red) measuring via CEAS during thermal decomposition of acetone behind reflected shock waves. Figure reprinted from [150] with permission from The Optical Society of America.

Similarly, interband and quantum cascade lasers have

2210 helped expand the list of fuel intermediates measureable in shock tubes to include acetylene [264, 265], propene [109], and iso-butene [110].

\subsubsection{Cavity-Enhanced Studies}

As discussed in Section 4.4, optical cavities can be to improve the sensitivity of an LAS measuremen by increasing the distance over which light interacts with the absorbing species. Increased sensitivity in the detection of a given gas species facilitates improved shock tube experiments in two primary ways. First, experiments may be more highly diluted with an inęrt bath gas such that the test time involves dampened transients of temperature and pressure when reaction occurs. Dampened transients, especially temperature, reduce experimental uncertainties. Second, enhanced

2225 sensitivity allows for the detection of certain species that may have been considered infeasible over the typical inner diameter of a shock tube $(\approx 10 \mathrm{~cm})$.

Several variations of cavity-enhanced absorption have been deployed in shock tubes. Sun et al. has demonstrated low-finesse, off-axis integrated cavity output spectroscopy for carbon monoxide [150] and acetylene [151] detection in the infrared. Figure 24 shows the formation of $\mathrm{CO}$ from the thermal decomposition of only $10 \mathrm{ppm}$ acetone, thereby highlighting the

2235 ability of CEAS to provide high-fidelity species timgs histories in highly dilute gases. With a cavity-enhanced absorption gain factor of 91 , a detection limit of $\approx 100$ ppb of $\mathrm{CO}$ was achieved at combustion temperatures using a near-IR telecommunication-grade TDL. Addi2240 tionally, Alquaity et al. [266] utilized a pulsed QCL near $4.6 \mu \mathrm{m}$ in a cavity-ringdown spectroscopy technique for sensitive measurements of ethylene. Both 2 f these techniques involve replacing the shock tube win- dows with mirrors that are partially transmissive. Another technique, intra-cavity absorption spectroscopy (ICAS), does not require high reflectivity mirrors but rather involves gas detection within the cavity of a broadband laser. Fjodorow et al recently demonstrated this approach to measure acetylene near $1.5 \mu \mathrm{m}$ in a shock tube using a home-made erbium fiber laser [267].

All of these cavity-enhanced techniques require more complex alignment than conventional LAS and take advantage of the relatively quiescent environment behind a reflected shock wave. As such, their extension to more harsh combustion conditions has promise, but comes with additional practical challenges.

\subsection{Laboratory Flames}

Laboratory flames, ranging from low-pressure flatflame burners to high-pressure flow reactors, are used for the study of combustion chemistry primarily by assessing the variation of species and gas properties in space (along the axis of flow), as opposed to time for shock tubes. Historically, species measurements in flames have typically been acquired using gas chromatography (GC) and mass spectrometry (MS) methods coupled with sampling probes. A number of chemistry studies using low-pressure burners have also leveraged ultraviolet laser absorption and fluorescence measurement techniques, but the implementation of IRLAS has been sparse. A few recent efforts are discussed here.

Similar to shock tube studies, temperature is a critical parameter for interpreting the chemistry governing flames. Li et al. [268] introduced an $\mathrm{H}_{2} \mathrm{O}$ two-line thermometry technique near $2.9 \mu \mathrm{m}$ for low-pressure flames and demonstrated this with measurements of spatially resolved temperature and $\mathrm{H}_{2} \mathrm{O}$ concentration in methane-fueled flames. To minimize the influence of line-of-sight non-uniformities, sapphire rods were used to direct the light across a relatively uniform portion of the flame. Smith et al. [183] improved upon this thermometry technique by implementing scannedWMS spectral fitting and by using two sapphire rods cleaved at $45^{\circ}$ to direct light in and out of the flame. More recently, Nau et al. [269] used a quantum cascade laser near $4.5 \mu \mathrm{m}$ to measure $\mathrm{CO}, \mathrm{CO}_{2}$, and temperature in $2 \mathrm{D}$ (via tomography) in fuel-rich methane flames. In addition to these studies, which provided insight into 1D-chemistry models, many other IR-LAS sensors have simply been demonstrated in flames. Such demonstrations have been omitted from this section.

\section{Sensors for Power Plants and Gasifiers}

LAS-based measurements are frequently needed to characterize the behavior of industrial-scale combustion systems since their harsh and often corrosive environment complicates the use of intrusive sensors (e.g., 

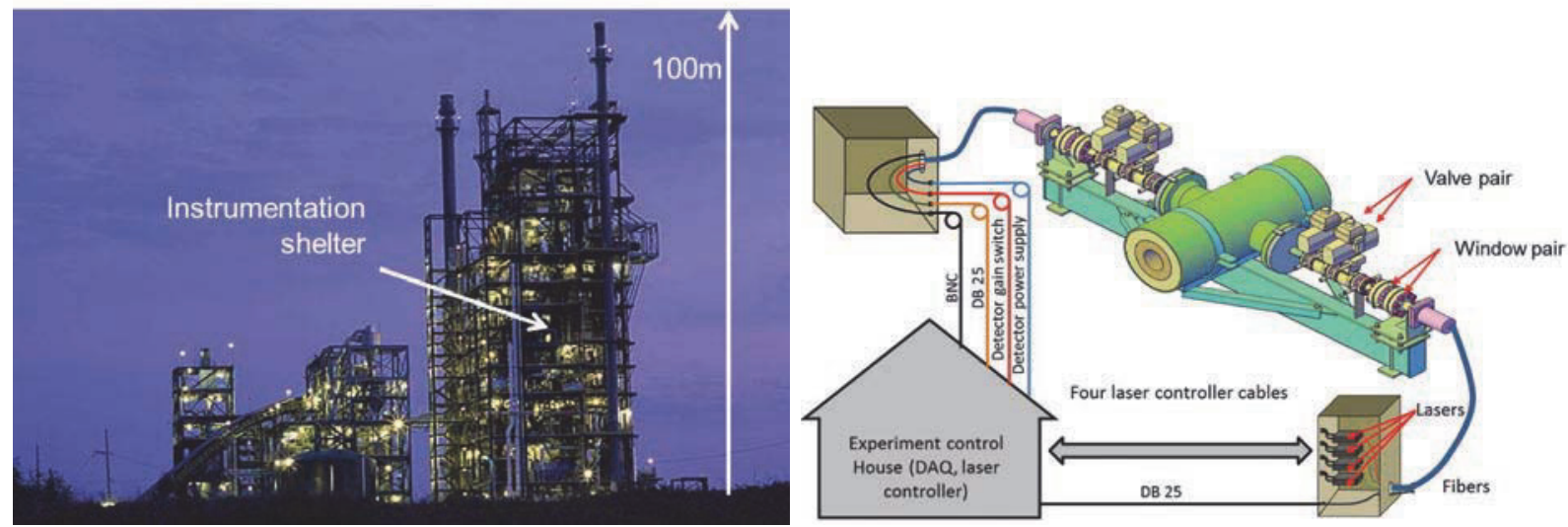

Figure 25: Photo of the circulating fluidized bed coal gasifier (right structure) and the coal processing and pulverization and preparation plant (left structure) at the National Center for Carbon Capture. The measurement station was located on the 6th floor on piping containing the flow of hot, pressurized gasifier products after fluidized bed particles were removed from the flow. Sketch showing the sensor installation on the process product delivery pipe. Laser light is delivered with a $2 \mathrm{~m}$ long fiber bundle, then collimated along a LOS across the gas flow, and focused onto a detector. All ADP was located remotely $(\approx 30 \mathrm{~m})$ in an instrument shelter. Figures adapted from [270].

thermocouples, sampling probes). As such, there is a long history of IR-LAS for such systems. To our knowledge the earliest TDLAS measurements in a large-scale industrial combustor were conducted by Ebert et al [271] who measured the concentration of $\mathrm{O}_{2}$ and $\mathrm{H}_{2} \mathrm{O}$ in a waste incinerator. This work was followed by TDLAS measurements of $\mathrm{O}_{2}, \mathrm{CH}_{4}, \mathrm{CO}_{2}$, and $\mathrm{H}_{2} \mathrm{O}$ in a natural-gas fired power plant [272] and $\mathrm{CO}$ and $\mathrm{H}_{2} \mathrm{O}$ such systems are maturing and Zolo Technology currently provides a commercial sensor for $\mathrm{H}_{2} \mathrm{O}, \mathrm{CO}, \mathrm{O}_{2}$, and $\mathrm{CO}_{2}$ along multiple paths, enabling tomographic reconstruction of the distribution of combustion products and gas temperature [274]. More recently, measurements of $\mathrm{NO}$ and $\mathrm{CO}$ were acquired in the exhaust of coal-fired boilers [275, 276]. These pioneering works identified many of the difficulties of field measurements in industrial-scale combustion applications. For exam-

2315 ple, combustion-driven power plants and glass furnaces operate continuously for months (or even years) precluding any opportunity to install new window ports or change engineering designs. Again, when the largescale combustor is heated to operating temperatures, the entire assembly (fire-box, boiler, exhaust ducts) all move significant distances by thermal expansion, and the firewalls were not designed to be mechanically stable requiring at least remotely driven beam alignment and at best an auto-alignment arrangement. Sin?23 much of this early work has already been reviewed $[277,3]$, now we will direct our focus to more recent work done in coal-fired power plants and gasifiers.

The biggest challenge for LAS sensing in coal-fired systems is to overcome the large non-resonant trans-

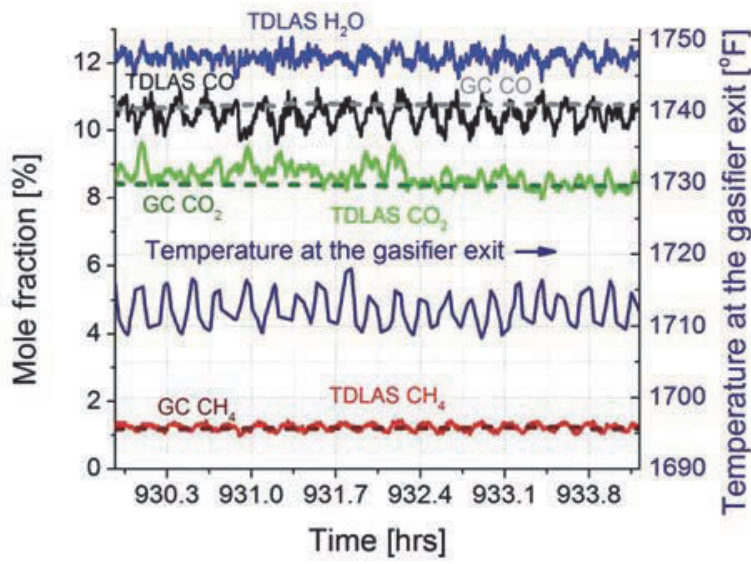

Figure 26: Forty minutes of time-resolved species mole-fractions $\left(\mathrm{CH}_{4}, \mathrm{CO}_{2}, \mathrm{CO}\right.$, and $\left.\mathrm{H}_{2} \mathrm{O}\right)$ from laser absorption sensor using scanned-WMS in the gasifier exit flow taken during the 39th day of operation; also plotted are sampled GC data for $\mathrm{CH}_{2}, \mathrm{CO}_{2}$, and $\mathrm{CO}$, as well as thermocouple temperature at the gasifier exit. Fluctuations in laser absorption data are not noise but follow actual fluctuations in the gasifier product mole fractions. Figures adapted from [270].

ticulates (e.g., soot, coal dust, ash). Recently, Sun et al. [278] demonstrated that this challenge could be overcome using WMS- $2 f / 1 f$ which enables calibrationfree measurements despite unknown levels of nonresonant extinction (as discussed in Section 4.3 and 4.5.1). The authors demonstrated measurements of temperature and $\mathrm{H}_{2} \mathrm{O}$ in a university-scale coal gasifier despite losing $99.99 \%$ of the laser light to particulate scattering.

Another challenge complicating LAS measurements in gasifiers is their high-operating pressures (20 to 50 atm) and the widely variable composition of the prod- 
uct gas. This severely complicates the modeling of absorption spectra and, therefore, quantitative interpre-

2345 tation of WMS- $2 f / 1 f$ signals. Recently this challenge was overcome by Sur et al. [270] by using the scannedWMS simulation and spectral fitting techniques developed by Sun et al. [131] and Goldenstein et zxtpo [132]. By determining the line broadening in situ, these methods enabled quantitative, calibration-free measurements of $\mathrm{CO}$ near $2326 \mathrm{~nm}, \mathrm{CO}_{2}$ near 2017 $\mathrm{nm}, \mathrm{CH}_{4}$ near $2290 \mathrm{~nm}$, and $\mathrm{H}_{2} \mathrm{O}$ near $1352 \mathrm{~nm}$ in the product stream (just downstream of the circulatos ing fluidized bed reactor as marked on the photo on

2355 the left side of Figure 25) of an engineering-scale coal gasifier [270] at the National Carbon Capture Center in Wilsonville, AL. This facility run by the Southern Company for DoE is meant to explore the feasibility of commercial-scale coal gasification for power generationto Figure 25 illustrates the size of the facility including the coal-pulverization plant and the gasifier facility. The TDLAS sensor architecture is illustrated on the right side of Figure 25. The automatic data processing and the laser control electronics were located $30 \mathrm{~m}$ away

2365 from the measurement location and the lasers were located within $2 \mathrm{~m}$ of the window ports due to the lack of quality fiber for the extended NIR wavelengths (2017, 2290 , and $2326 \mathrm{~nm}$ ). Note that higher-quality optical fiber for this wavelength region is now available that 2370 would now allow the lasers to be located with their control electronics (30 m from measurement location). Light from the four lasers was combined into a fiber bundle, collimated in free-space and pitched from one side of the process pipe to the other. The four laseress were time-multiplexed to use a single detector and each laser was wavelength scanned and modulated.

The sensor was able to provide time-resolved tempêrature, $\mathrm{CO}, \mathrm{CO}_{2}, \mathrm{CH}_{4}$, and $\mathrm{H}_{2} \mathrm{O}$ concentration throughout a 54 day campaign (including a 18-day period of unattended operation) of relatively stable gasification. Typical results are illustrated in Figure 26. Note the oscillations in time observed for the product gas mole fractions. These unexpected oscillations were corres lated with the temperature in the reactor and were synchronous with the batch feeder for pulverized coal injection. The average values of $\mathrm{CO}, \mathrm{CO}_{2}$ and $\mathrm{CH}_{4}$ measured by the laser absorption sensor were in good agreement with extractive gas chromatography (GGa), however, the extractive gas analysis was delayed by $2390 \approx 20$ minutes and had $\mathrm{a} \approx 15$ minute time response due to the transport of the sampled gas and diffusion in the large conditioning volume (filtering and drying) before the GC. The delay of the sampling system required for GC measurements highlights an advantage of LAS

\section{Sensors for Gas Turbines}

Several researchers have developed LAS sensors employing a variety of light sources for gas temperature and non-uniformities therein, $\mathrm{H}_{2} \mathrm{O}, \mathrm{CO}_{2}$, and $\mathrm{CH}_{4}$ in model gas-turbine combustors. The majority of work done to date has focused on characterizing the fuel loading and combustion processes relevant to lean blowout (LBO) via single LOS-measurements, and this section will focus on discussing the measurement science and technology enabling these works.

\subsection{Direct Absorption}

As already discussed, Seitzman and Scully [174] developed a strategy using a spectrally broad light source near $2.5 \mu \mathrm{m}$ for measuring temperature uniformity and $\mathrm{H}_{2} \mathrm{O}$ concentration at the exit of gas turbine combustors, however no demonstration measurements were presented. Later, this strategy was implemented using diode laser absorption of three $\mathrm{H}_{2} \mathrm{O}$ transitions near 2 $\mu \mathrm{m}$ to provide open-loop control of temperature nonuniformities at the exit of a stratified methane-air combustor [177]. Meyer et al. [279] developed a diode laserbased mid-IR source using difference-frequency mixing and demonstrated its use with measurements of $\mathrm{CO}_{2}$ near $4.5 \mu \mathrm{m}$ in the exhaust of a liquid-fueled model gas-turbine combustor. The authors demonstrated a detection limit of $44 \mathrm{ppm}$ and measurement rates up to $10 \mathrm{kHz}$. Regarding TDLAS sensors, Liu et al. [280] developed a TDLAS sensor for measurements of $\mathrm{H}_{2} \mathrm{O}$ and temperature via absorption of two $\mathrm{H}_{2} \mathrm{O}$ transitions near $1.4 \mu \mathrm{m}$. This sensor used time-division multiplexing and scanned-wavelength direct absorption, and was demonstrated in the exhaust of an industrial-scale gas turbine coupled to a $20 \mathrm{MW}$ electric generator. Data processing was performed in real-time to provide temperature measurements at $3 \mathrm{~Hz}$. The sensor was validated in controlled static-cell experiments with an accuracy of 2 to $5 \mathrm{~K}$ at temperatures from 350 to 1000 $\mathrm{K}$.

\subsection{Hyperspectral-DA}

Kranendonk et al. [38] used an FDML laser spanning 1330 to $1380 \mathrm{~nm}$ to measure gas temperature at $200 \mathrm{~Hz}$ (after averaging 1000 scans at $200 \mathrm{kHz}$ ) via $\mathrm{H}_{2} \mathrm{O}$ absorption in a gas-turbine combustor. Measurements were acquired at equivalence ratios and pressures from 0.22 to 0.32 and 3.4 to 6.9 bar, respectively, $6 \mathrm{~cm}$ downstream of the brightest region of the JP8-fueled combustor. The sensor revealed steady-state temperatures within 50 to $100 \mathrm{~K}$ of the semi-empirical flame temperature (derived from a combination of exhaust gas composition measurements and chemical equilibrium calculations) for each condition studied. The authors note that a significant aspect of this work was demonstrating 
a

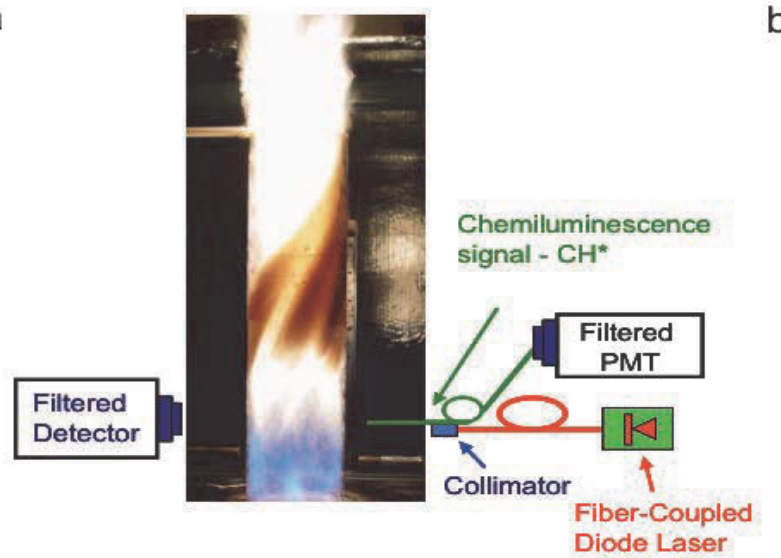

b

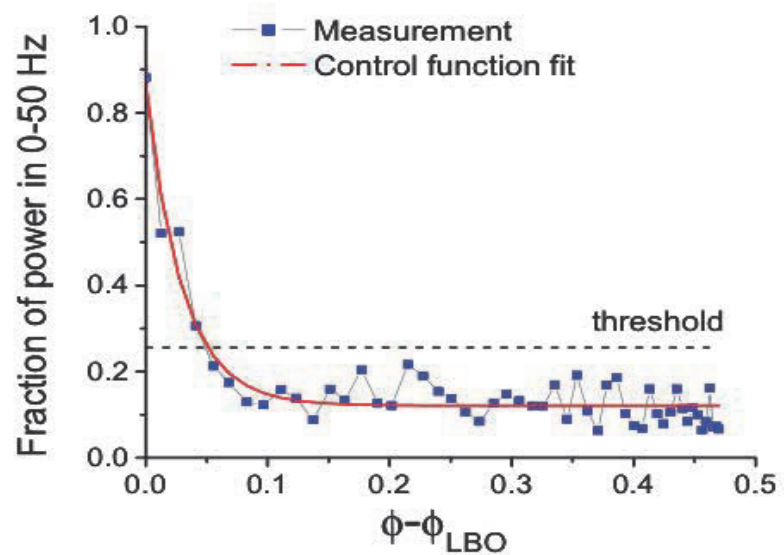

Figure 27: Experimental setup used to monitor lean-blowout via TDLAS (a) and frequency content in temperature signal as a function of differential equivalence ratio (b). Reprinted from [3] with permission from Elsevier.

the sensor's ability to tolerate the bright background emission observed in the gas-turbine combustor compared to previous combustion studies performed using FDML lasers (e.g., [36, 37]).

Later, Caswell et al. [281] developed two light sources using fiber Bragg gratings for measuring gas temperature, $\mathrm{H}_{2} \mathrm{O}$, and $\mathrm{CH}_{4}$ in a high-pressure gasturbine combustor. In comparison to diode laser- and FDML-based sensors, the authors point out that these light sources can provide absorption information at multiple discrete wavelengths over a wider spectral range (between 1329 and $1667 \mathrm{~nm}$ here) at high rates $(30 \mathrm{kHz})$. This sensor employed 4 discrete wavelengths to yield $\mathrm{H}_{2} \mathrm{O}$ concentration and optimal thermometry performance from 500 to $2500 \mathrm{~K}$ and at pressures from 3 to 30 bar, 4 wavelengths to monitor linebroadening near the wavelengths for thermometry, and two nonresonant wavelengths on the extreme ends of the spectral bandwidth to infer $I_{o}$. Regarding $\mathrm{CH}_{4}$ sensing, 4 additional wavelengths near $1665 \mathrm{~nm}$ were employed to monitor temperature-sensitive and -insensitive $\mathrm{CH}_{4}$ absorption, pressure broadening, and inferring $I_{o}$. By detecting only discrete wavelengths, a sample rate of only $22 \mathrm{MHz}$ was needed, which is approximately 10 times less than that required by FDML-based sensors of comparable measurement rates. Fiber optics were used to transmit and collect laser light pitched across the combustor inlet and exit and were intelligently designed to rid the need of user alignment. Time-resolved measurements of temperature and $\mathrm{CH}_{4}$ at the inlet, and temperature and $\mathrm{H}_{2} \mathrm{O}$ at the exit are presented at temperatures up to $1700 \mathrm{~K}$ and a pressure near 10 bar.

Most recently, Ma et al. [189] developed and deployed a hyperspectral tomographic sensor for reconstructing the 2D temperature and $\mathrm{H}_{2} \mathrm{O}$ concentration field in the exhaust plane of a General Electric J aero-propulsion engine. This work employed 32 fiber-
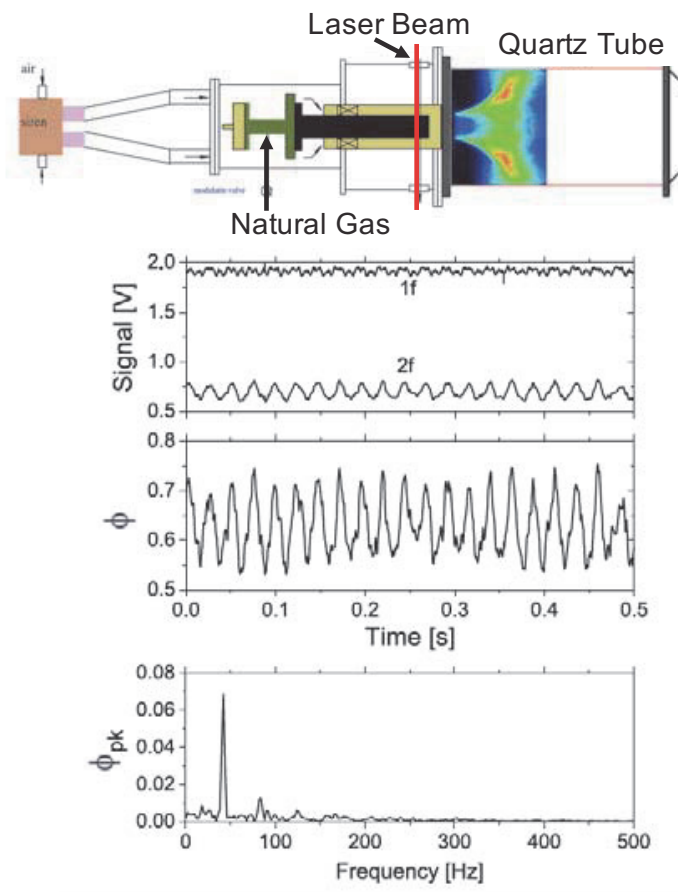

Figure 28: Schematic of experimental setup used to measure equivalence ratio in a model gas-turbine combustor and example results during a modulated-fuel experiment. Figures adapted from $[282]$.

coupled laser beams and used 12 wavelengths to recover the 2D fields on a 225-pixel grid at $50 \mathrm{kHz}$. More details can be found in the review by Bolshov et al. [8] and the original manuscript [189].

\section{3. $W M S$}

Several WMS-based sensors have been developed for studying lean-blowout in gas-turbine combustors. 
Zhou et al. [283] developed diode-laser-based sensors for real-time (onboard post-processing) measurementas of gas temperature and $\mathrm{H}_{2} \mathrm{O}$ in a swirl-stabilized model gas-turbine combustor. Two single-laser sensors were developed, one near $1.8 \mu \mathrm{m}$ using scanned-DA and one near $1.4 \mu \mathrm{m}$ using WMS-2f. The WMS-based sensor provided measurements of gas temperature at $2 \mathrm{~kb} 50$ and demonstrated higher-SNR (compared to direct absorption) in flowfields with soot or droplet scattering. Later, Li et al. [284, 285] extended the WMS- $2 f$ sensor near $1.4 \mu \mathrm{m}$ to be the first laser-based sensor for active lean-blowout control of swirl-stabilized flames ím a model gas-turbine combustor. The authors found

2505 that by monitoring the fractional power contained in the FFT (of temperature) between 0 and $50 \mathrm{~Hz}$, leanblowout could be predicted and prevented using an active control system. This enabled continuous flang holding at equivalence ratios only 0.03 higher than the LBO condition. Fig. 27 shows a schematic of the experimental setup used and the power-spectrum of the temperature time-history as a function of the difference between the equivalence ratio and the LBO value. ${ }^{2565}$ Using similar technology, Li et al. [282] later de2515 veloped a WMS- $2 f$ based TDL sensor for measuring $\mathrm{CH}_{4}$ using the $\mathrm{R}(3)$ line near $1653.7 \mathrm{~nm}$. These measurements enabled determination of the equivalence ratio of the gas entering a model gas-turbine combustign chamber in real time. By measuring the equivalence

2520 ratio in a modulated fuel experiment, the authors were able to correlate oscillations in equivalence ratio with that of heat release, and used the results as a direct input into an analytical model for predicting thermobrs coustic instability and LBO. Fig. 28 shows a schematic

2525 of the experimental setup used and time-resolved measurements of the equivalence ratio calculated from the WMS- $f$ signal. The results shown highlight the oscillations in equivalence ratio (induced by modulating the fuel flow rate) of the gas entering the combustor,

2530 and the FFT reveals that the oscillation occurs at near $50 \mathrm{~Hz}$.

\section{Sensors for IC Engines}

As discussed in Section 6.4, several researchers have developed LAS sensors integrated into spark plugs [248,

$2535249,42,250,241]$. Since these sensors have already been discussed, this section will focus on non-intrusive line-of-sight sensors for measurements of gas properties in IC-engine systems.

\subsection{Fixed-Wavelength DA}

Fixed-wavelength direct absorption has been used to study IC engines due to 3 primary advantages: 1) exas tremely fast time response $(>1 \mathrm{MHz})$ limited by the sampling rate and detector bandwidth only, 2) simple and computationally efficient data processing, and 3) tolerance to non-wedged windows common to optical engines. Mattison et al. [123] developed a three-color fixed-wavelength fiber-coupled sensor for two HCCI optical engines. The sensor used three DFB diode lasers to monitor $\mathrm{H}_{2} \mathrm{O}$ absorption near 7219.61 and 7416.05 $\mathrm{cm}^{-1}$ and non-resonant transmission near $7606 \mathrm{~cm}^{-1}$. A diffraction grating was used to demultiplex the colors onto three separate detectors. This sensor was demonstrated with measurements of temperature and $\mathrm{H}_{2} \mathrm{O}$ during motored- and fired-operation at temperatures and pressures up to $1750 \mathrm{~K}$ and near 50 atm, respectively. The combination of variable pressure and pressure-dependent birefringence of the sapphire windows made it important to minimize polarizationdependent transmission fluctuations. During motored operation the sensor recovered the semi-empirical temperature (computed from known cylinder volume and measured pressures) within 3\%. During fired operation the sensor recovered the semi-empirical temperature within $5 \%$, the increased error was primarily attributed to LOS non-uniformities.

Several researchers have also developed tomography based LAS sensors for time-resolved, in-cylinder imaging of hydrocarbon fuels in single- [286] and multicylinder engines [287]. In the latter, trenches for fiberoptics were machined into the engine block to enable fiber-optic light delivery and collection along 27 LOS. Two diode lasers at 1700 and $1651 \mathrm{~nm}$ were frequencymultiplexed at 300 and $500 \mathrm{kHz}$ to enable simultaneous monitoring of hydrocarbon absorption and nonresonant extinction. The authors presented qualitative images of fuel concentration at 3 to $4 \mathrm{k}$ frames per second, and note that the system provided signal on all beams for $>2$ hours of fired operation.

\subsection{Scanned-DA}

Witzel et al. [288] developed a scanned-wavelength DA sensor using a tunable diode laser near $1370 \mathrm{~nm}$ for measuring $\mathrm{H}_{2} \mathrm{O}$ concentration at $4 \mathrm{kHz}$ during the compression stroke of an optically accessible IC-engine. Measurements were acquired at temperatures and pressures from 300 to $500 \mathrm{~K}$ and 0.7 to 5.2 bar. The laser light was transmitted through the quartz cylinder offaxis to minimize etalon fringes. Later, this sensor was improved upon by using a VCSEL near $1370 \mathrm{~nm}$ to provide greater scan amplitude and time resolution (10 $\mathrm{kHz}$ ) [165]. By using a VCSEL the entire $\mathrm{H}_{2} \mathrm{O}$ absorption line could be resolved which improved measurement precision by nearly a factor of 2 compared to previous work done with the TDL. Most recently, Ebert et al. [247] integrated the aforementioned diodelaser-based sensor into a production 4-cylinder engine with minimal engine modifications to provide measurements of $\mathrm{H}_{2} \mathrm{O}$ concentration at $4 \mathrm{kHz}$. This sensor used 

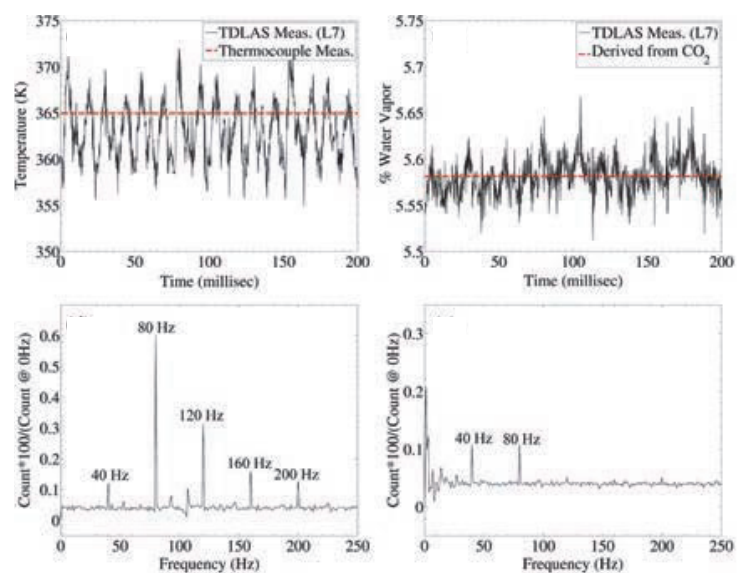

Figure 29: Measured time history and FFT of gas temperature and $\mathrm{H}_{2} \mathrm{O}$ mole fraction acquired at the exit of an exhaust-gas recirculation (EGR) cooler using LAS. Figures adapted from [125].

a SMF transmitter mounted to the engine to pitch light across the cylinder. A probe with a $45^{\circ}$ mirror was used (i.e., upwards) into a 600 m MM. The authors used a transmission levels to only $1 \%$, thereby prohibiting the use of the much lower power VCSEL-based sensor described in [165]. Using this sensor the authors were able to achieve a detection limit as low as $7400 \mathrm{ppm}$ of $\mathrm{H}_{2} \mathrm{O}$ by volume.

Recently, Jatana et al. [289, 125] developed a TDL sensor for simultaneous temperature, $\mathrm{H}_{2} \mathrm{O}$, and pressure in the intake manifold, EGR cooler exit, and turbo-charger inlet of a diesel engine. The sensor used a single DFB TDL near $1388 \mathrm{~nm}$ and custom fabricated mounts for mounting the photodetectors and SMF transmitters to the engine. The mounts utilized a ce-

2615 ramic spacer to insulate the optical equipment from the high-temperature (up to $850 \mathrm{~K}$ ) components without needing water cooling. By using telecommunicationgrade fibers and splitters this sensor was able to acquire measurements at 4 locations simultaneously. During operation, the laser was scanned across $6 \mathrm{H}_{2} \mathrm{O}$ transitions (4 dominant), with $E$ " ranging from 70 to 1742 $\mathrm{cm}^{-1}$, at up to $5 \mathrm{kHz}$. Gas properties were inferred from a spectral-fitting routine. Fig. 29 shows example temperature and $\mathrm{H}_{2} \mathrm{O}$ results. The sensor was able to $\mathrm{H}_{2} \mathrm{O}$ mole fraction from 1.4 to $4 \%, 5.5$ to $6.5 \%$, and 6.2 to $8.2 \%$, respectively, for temperatures from 300 to $850 \mathrm{~K}$ with measurement precisions ranging from 0.9 to $4 \%$.

\subsection{Hyperspectral-DA}

Kranendonk et al. [37] used an FDML laser with a linewidth of $0.1 \mathrm{~nm}$ for measurements of gas tem-

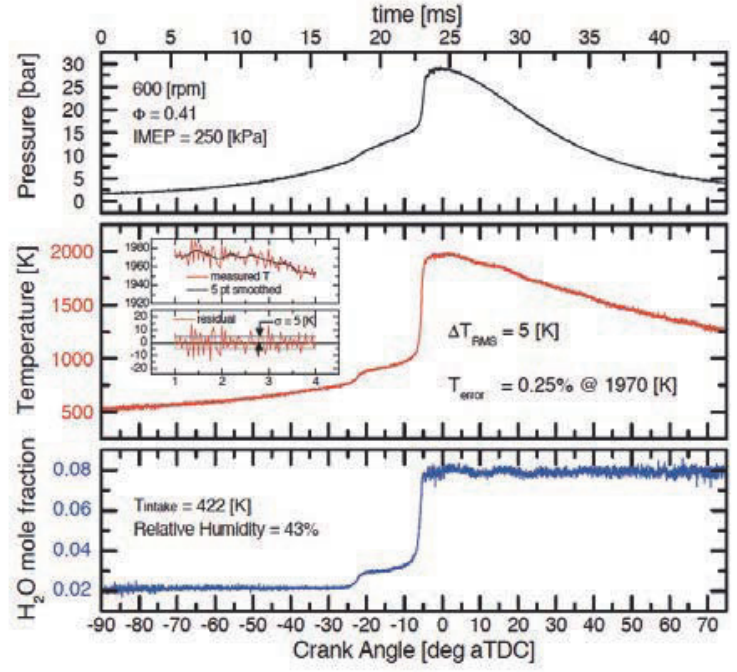

Figure 30: Example results of temperature and $\mathrm{H}_{2} \mathrm{O}$ measured in an HCCI optical engine using a FDML laser. Figure reprinted from [210] with permission from The University of Wisconsin.

perature in an HCCI engine at $100 \mathrm{kHz}$ and temperatures and pressures ranging from 300 to $800 \mathrm{~K}$ and 2 to 18 bar. The laser interrogated the $\mathrm{H}_{2} \mathrm{O}$ absorption spectrum from 1335 to $1373 \mathrm{~nm}$ and the methods presented in [290] were used to calculate gas properties from the measured spectra. Optical access was provided through two sapphire windows located in pressure-transducer ports machined into the pent-roof combustion chamber. Later, Caswell [210] extended the use of this FDML laser to simultaneous measurements of temperature and $\mathrm{H}_{2} \mathrm{O}$ at up to $2000 \mathrm{~K}$ and 30 bar in a fired HCCI engine (see Figure 13). Fig. 30 shows sample results indicating an impressive RMS temperature error of only $5 \mathrm{~K}$ which, to the best of our knowledge, still represents the best precision achieved in IC-engine thermometry. Gas properties were calculated using the methods described in [127] and the BT2 line list [88]. To overcome the fact that BT2 does not contain collisional-broadening parameters, an algorithm was developed to determine best-fit broadening coefficients for several spectral windows, since collisional broadening, for $\mathrm{H}_{2} \mathrm{O}$, is highly dependent on the transition. While this technique does not attempt to resolve the broadening-coefficients of each individual transition (recognizing that this would be impractical), it was found to improve results significantly compared to previous algorithms that assumed equal line broadening for all transitions.

\section{4. $W M S$}

To the best of our knowledge, WMS has currently only been used to study production-grade IC-engines via the spark-plug-based probes discussed in Sect. 6.4.1 
2665 and $[42,241]$. In comparison to the works discussed previously in this section, these sensors were forced to overcome short optical path lengths (5 to $12 \mathrm{~mm}$ ) to provide time-resolved measurements of gas temperature, $\mathrm{H}_{2} \mathrm{O}$ or gasoline mole fraction.

\section{Sensors for Scramjets}

Many researchers have developed LAS sensors for variety of high-speed combustion flows [239, 240, 291, 178, 292, 41, 181, 293, 243, 294, 184, 219, 244]. Among the most frequently studied applications in this cateare hypervelocity shock tunnels $239,240,295]$ ramjets, and scramjets $[291,178,292,41,181,293,28$ 124, 188, 243, 294, 244]. Early work was conducted in shock tunnels to characterize their hypersonic flow fields [239, 240] and similar efforts continue [295]. Of late, most LAS sensing in high-speed flows has been done to characterize scramjet flow fields. The scramjest represents one promising propulsion technology capable of providing airbreathing hypersonic flight with no moving engine parts. The scramjet consists of four

2685 primary components: inlet, isolator, combustor, and nozzle. The inlet slows the incoming hypersonic axi through an oblique shock-train, raising the temperature and pressure of the gas and the isolator is used to homogenize the flow field entering the combustor.

${ }_{2690}$ Fuel is directly injected into the high-temperature air stream to initiate combustion and the combustion gases are expanded in the nozzle to produce thrust. During operation, the goal is to combust enough fuel to produce sufficient thrust while avoiding unstart (i.e., a

2695 failure mode where the shock train is expelled from the isolator yielding subsonic flow throughout the scramjest flow path). As a result, the performance of a scramjet requires a delicate balance between a variety of complex physical process, namely, fuel-air mixing, chemical

2700 kinetics, and shock-boundary-layer interaction. The need to understand these processes in situ has driven the development and application of LAS sensors for characterizing scramjets.

Historically, most IR-LAS sensing in scramjets has employed near-IR telecommunication-grade TDLs for measuring temperature, $\mathrm{H}_{2} \mathrm{O}$, and velocity continuous-flow facilities [291, 178, 43, 293, 124, 188, 296, 297], with only one prior example of measuring $\mathrm{CO}_{2}$ in [292] (later discussed in [41]). Recently, sev-

2710 eral efforts have extended sensing capabilities to: 1) the mid-infrared for improved measurements of $\mathrm{H}_{2} \mathrm{6}$ $[184,294], \mathrm{CO}_{2}[219,294]$, and the first measurements of CO $[219,294]$ in a hydrocarbon-fueled model scramjet combustor, 2) 2D via tomography [124, 190, 126],

$27153)$ greater bandwidth for improved sensing in impulse facilities [244, 159], and 4) the first in-flight TDLAd measurements for characterizing scramjet flow fields $[26,27,28,298]$.

\subsection{Sensors for Continuous-Flow Facilities}

The recent combined effort presented in Goldenstein et al. [184], Spearrin et al. [219], and Schultz et al. [294] represents the first demonstration of midinfrared LAS sensing of $\mathrm{H}_{2} \mathrm{O}, \mathrm{CO}_{2}$, and $\mathrm{CO}$ in scramjets, made possible by the advent of thermoelectrically cooled quantum-cascade lasers and mid-infrared fibers. Furthermore, this work represents one of the first applications of calibration-free scanned-WMS spectral fitting and the use of the two-color strategy for acquiring path-averaged gas properties in highly non-uniform flows (discussed in Section 5 and [171]). The combination of these strategies was critical to the acquisition of accurate measurements due to the large temperature and composition gradients within the scramjet combustor studied.

Fig. 31a illustrates a schematic of the model scramjet studied (the University of Virginia's Supersonic Combustion Facility (UVaSCF)) and the orientation of the translating lines-of-sight used by all sensors. Each laser was fiber-coupled in free-space into a mid-IR fiber (multi-mode ZBLAN for $\mathrm{H}_{2} \mathrm{O}$, bifurcated hollow core for $\mathrm{CO}$ and $\mathrm{CO}_{2}$ ) and an $\mathrm{X}-\mathrm{Y}$ translation stage was used to translate the measurement line-of-sight throughout the combustor. DFB TDLs near 2482 and $2551 \mathrm{~nm}$ were used to measure $\mathrm{H}_{2} \mathrm{O}$ and temperature via scanned-WMS-2f/1f, a DFB QCL near $4855 \mathrm{~nm}$ was used to measure $\mathrm{CO}$ and temperature via scannedDA and scanned-WMS- $f f / 1 f$, and an external-cavity QCL near $4176 \mathrm{~nm}$ was used to measure $\mathrm{CO}_{2}$ via scanned-DA. The use of stronger fundamental-band absorption enabled access to transitions that are 20 to $10^{4}$ times stronger than in the near-IR (depending on species) which improves measurement sensitivity and enables use of inherently weaker transitions with greater lower-state energy for improved temperature sensitivity and tolerance to thermal non-uniformities [74]. However, these benefits do not come without challenges, namely that use of wavelengths beyond $4 \mu \mathrm{m}$ in a high-temperature, continuous-flow facility complicates the use of sapphire windows (leading to signifcant thermally induced temporal fluctuations in this work) and other less robust materials (e.g., ZnSe) that oxidize in high-temperature combustion gases. More information regarding these complications can be found in Section 6.2.4.

Fig. 31b shows time-averaged species measurements as a function of the $x$-direction (transverse to flow) for two measurement planes. By comparing the results in the top panel (downstream plane) to the bottom panel (upstream plane) it is clear that the combustion products expand into the freestream. Furthermore, at loca- 

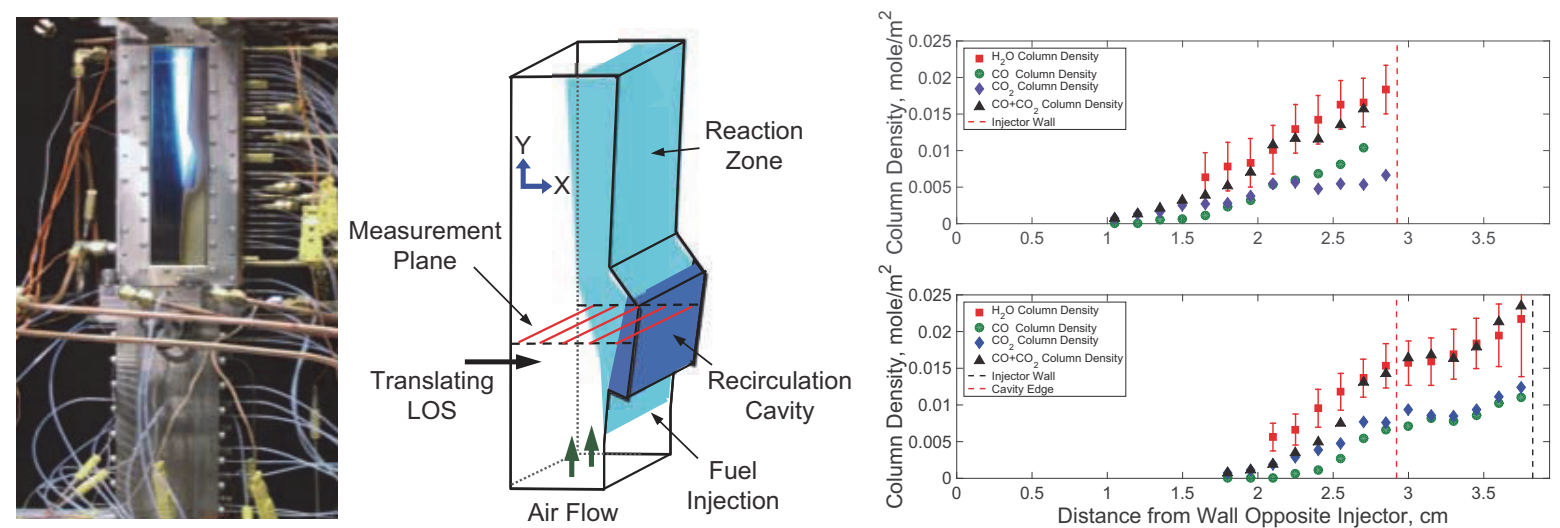

Figure 31: Schematic of ethylene-fueled, model-scramjet combustor studied using mid-IR LAS and time-averaged species concentrations as a function of space. Figures adapted from [219] and [294].

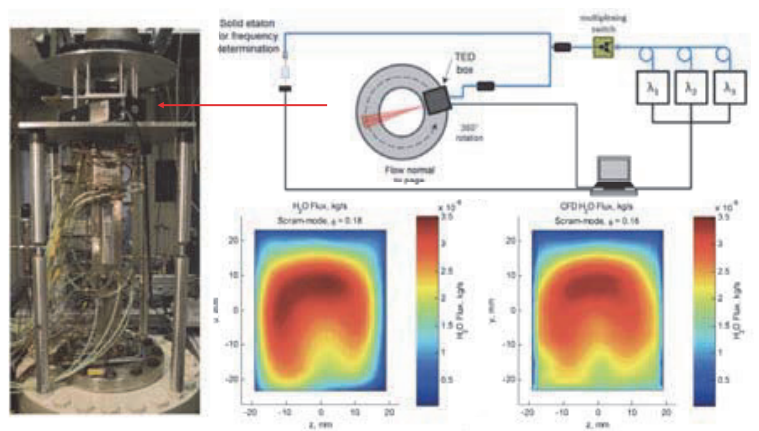

Figure 32: Schematic showing UVaSCF, experimental setup of TDLAT sensor, and comparison of measured $2 \mathrm{D} \mathrm{H}_{2} \mathrm{O}$ flux with CFD. Figures adapted from. [126].

tions near the flame-holding cavity the column-density of $\mathrm{CO}+\mathrm{CO}_{2}$ agrees remarkably well with that of $\mathrm{H}_{2} \mathrm{O}$ indicating that most of the carbon and hydrogen atorins originally contained in the fuel $\left(\mathrm{C}_{2} \mathrm{H}_{4}\right)$ have moved to tions near the high-speed free-stream (i.e., far from the cavity), the column density of $\mathrm{CO}+\mathrm{CO}_{2}$ is significantly less compared to $\mathrm{H}_{2} \mathrm{O}$ indicating the presence of uppe burned fuel/hydrocarbons at this location in the flow. These results enable a variety of interesting conclusions regarding the spatio-temporal evolution of combustion progress that would not be possible without acquiring multi-species measurements.

2805

Brown et al. [124] demonstrated the use of a tun2785 able diode-laser absorption tomography (TDLAT) sensor for coarse reconstruction of temperature and $\mathrm{H}_{2} \mathrm{O}$ at the exit of a ground-test scramjet designed to replicate the HIFiRE 2 scramjet. This sensor employerd three time-multiplexed diode lasers near $1.4 \mu \mathrm{m}$ along 14 lines-of-sight (8 vertical, 6 horizontal). Algebraic reconstruction tomography (ART) was used to reconstruct the 2D distribution of absorbance for each color

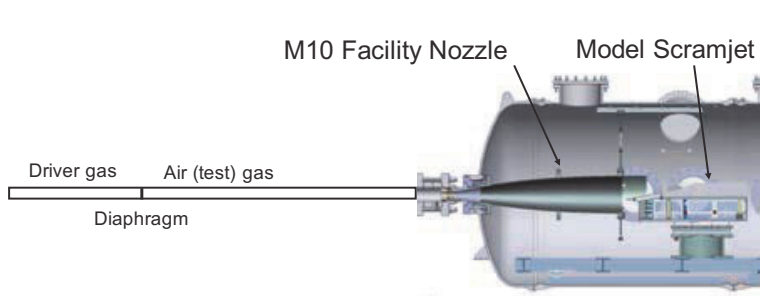

Figure 33: Schematic of the NASA hypersonic pulse (HyPulse) reflected shock-tunnel facility (operated by ATK General Applied Science Laboratory (GASL)) used for scramjet testing with TDLAS sensors. For more details see [243].

since it is more suitable than filtered back projection techniques in cases with a limited number of LOS measurements. After the 2D integrated absorption of each color was reconstructed, the gas temperature and water mole fraction were calculated in a $9 \times 19$ pixel image using standard LAS techniques. During steady-state operation, the 2D distributions of $\mathrm{H}_{2} \mathrm{O}$ and temperature indicate burning in the core of the flow with local hotspots and the distributions were found to vary substantially in time, indicating the role of turbulent combustion.

Busa et al. [188] documented the design and initial implementation of a TDLAT system for 2D temperature and $\mathrm{H}_{2} \mathrm{O}$ concentration at NASA Langley's directconnect supersonic combustion test facility (DCSCTF) employing a fan-beam geometry and retroreflector to return the transmitted light to the pitch/catch optics. A rotational stage was used to rotate 5 separate transmitter/reciever boxes 72 degrees for an effective rotation of 360 degrees. Most recently, Busa et al. [126] reported the use of TDLAT and stereoscopic PIV for determination of combustion efficiency via spatially re- 

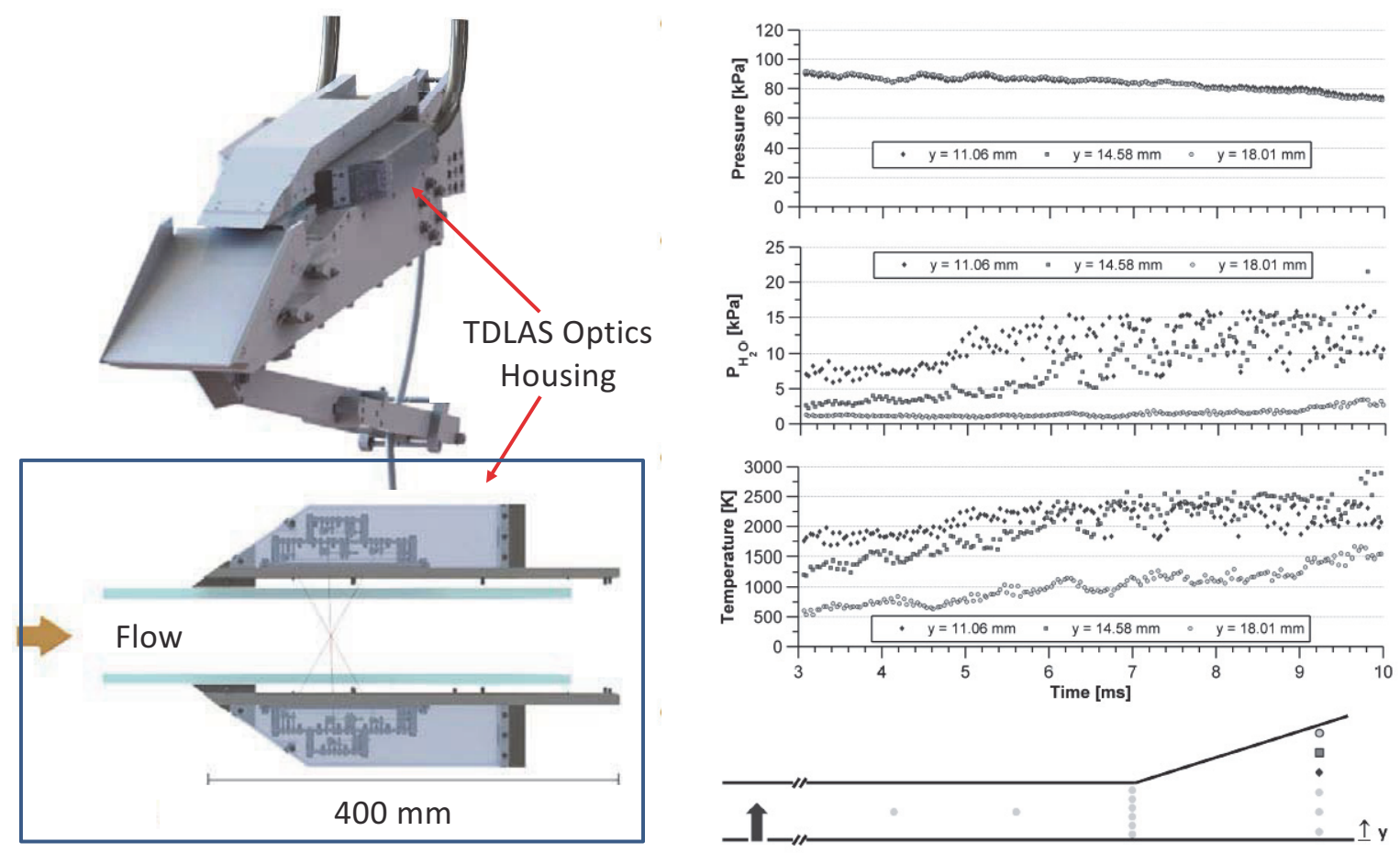

Figure 34: Model of HyShot II scramjet with TDLAS sensor and example of time-resolved temperature and $\mathrm{H}_{2} \mathrm{O}$ acquired via scanned-WMS- $2 f / 1 f$ during an engine-fired test. Figure adapted from [159].

\subsection{Sensors for Impulse Facilities}

Shock tunnels have been used to generate shortduration hypersonic flows with flight-enthalpies (typically $>$ Mach 5) that are too extreme to sustain in 2830 continuous-flow ground-test facilities. In practice, such facilities shock-heat gases to high-enthalpy conditionas and then expand them, for example, through a nozzle to generate hypersonic flow (see Figure 33). As a result, these facilities offer a unique opportunity to study scramjets in the laboratory at more extreme flight conditions. Shock tunnels pose a number of uniqua challenges including short test times (order of $1 \mathrm{~ms}$ ), transient flow conditions, and limited optical access. Despite these challenges, several researchers have developed TDLAS sensors for characterizing such tunnels [239, 240] and test articles housed within them $[299,243,244]$.

Two TDLAS measurement campaigns were recently conducted in model-scramjet combustors located within shock tunnels at ATK GASL [243] and the German Aerospace Center (DLR) [159]. Figure 34 shows a schematic of the experimental setup used and example results for a single test. Both sensors utilized DFB TDLs near $1.4 \mu \mathrm{m}$ with scanned-WMS- $2 f / 1 f$ to measure gas temperature and $\mathrm{H}_{2} \mathrm{O}$ concentration along three lines-of-sight (at various locations depending on test) to quantify the evolution of combustion progress in space. In addition, the work conducted by Strand [159] employed angled beams for velocimetry. The two campaigns used similar optical hardware for light delivery and optical alignment. The fibers were housed in a protective pipe and the pitch and catch optics were located behind a protective shield to protect all components from the high-speed flow. The results shown in Figure 34 highlight the utility of high-speed $(25 \mathrm{kHz})$, multi-line-of-sight measurements for characterizing the 
combustion process. The measurements indicate relatively stable combustion, but with some strong-, and rapid- (time-scale of $1 \mathrm{~ms}$ ) fluctuations in temperature and $\mathrm{H}_{2} \mathrm{O}$ despite a temporally stable wall-pressure. The TDLAS measurements also reveal a strong bias of combustion products towards the fuel-injector wall.

\subsection{Sensors for In-Flight Tests}

Brown et al. [28] presented the use of two independently developed TDLAS sensors for onboard measurements of $\mathrm{O}_{2}$ in a hypersonic vehicle as part of the HIFiRE I test campaign. The sensor developed by Zolo Technologies used scanned-wavelength direct absorption [26] while the sensor developed by Southwest Sciences used scanned-WMS [27]. Both sensors employed angled lines-of-sight across mock-inlet flow paths to infer the flow velocity from the Doppler-shifted absorption, with the ultimate goal of measuring $\mathrm{O}_{2}$ mass flux entering the combustor. The scanned-DA sensor em-

2880 ployed a scan rate of $5 \mathrm{kHz}$ and multiple scans weres averaged to yield a measurement rate of $10 \mathrm{~Hz}$. The scanned-WMS sensor used a scan rate of $1 \mathrm{kHz}$ and averaged the output to a measurement rate of $1 \mathrm{~Hz}$. Both sensors used lasers near $760 \mathrm{~nm}$ and the WMS sensor also included an onboard reference cell for active laseen frequency stabilization and a reference signal for velocimetry. As expected, the measured WMS lineshapes exhibited a greater signal-to-noise ratio than those of DA. During the flight, both sensors maintained align2890 ment and operated as planned for 17 seconds, aftere which the telemetry system for the WMS sensor began to operate unreliably. While no quantitative results are reported, it is clear that this work represents an impressive and encouraging demonstration of stand-alone TDLAS sensors for characterizing hostile systems. 2930

More recently, in-flight TDLAS measurements of $\mathrm{H}_{2} \mathrm{O}$ and temperature were acquired along 8 lines-ofsight traversing the exit plane of the HIFiRE 2 scramjet combustor [298] (see Figure 35). Two independent 2900 systems were used, each employing a single DFB T20 ⿷匚 scanned across multiple $\mathrm{H}_{2} \mathrm{O}$ transitions near $1.4 \mu \mathrm{m}$ at $50 \mathrm{kHz}$. The authors note that both systems operated successfully in flight, and point the reader to papers discussing analogous ground-test experiments [124] for greater detail and example results.

\section{Sensors for Pulsed-Detonation Combustors}

The thermodynamic advantages of high-pressure combustion and desire to minimize moving parts combustion systems has driven the development of

2910 a variety of detonation-based combustors and engines (e.g., pulse-detonation engine (PDE), rotatingdetonation engine (RDE), etc.). The extreme and

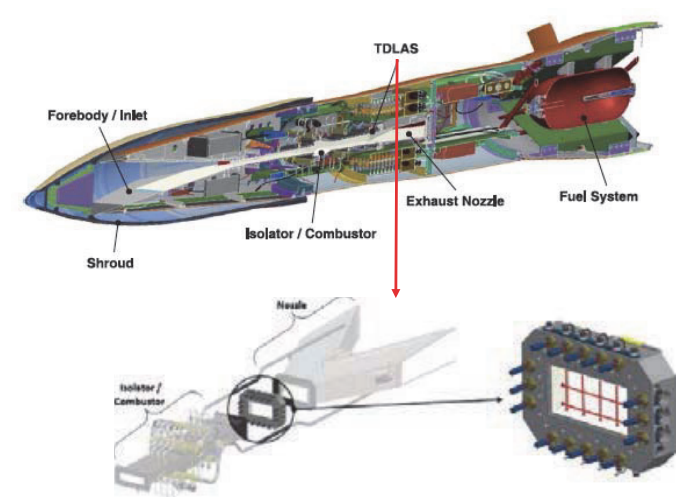

Figure 35: Schematic of the HIFiRE 2 scramjet and optical assembly used for TDLAS measurements of $\mathrm{H}_{2} \mathrm{O}$ and temperature in the scramjet's exhaust. Figure adapted from [298].

highly transient gas conditions produced by detonation combustors present one of the most challenging environments for LAS sensors. Such devices are capable of producing temperatures and pressures in excess of $4000 \mathrm{~K}$ and 100 bar, respectively, with changes in temperature and pressure of comparable magnitude occuring on $\mu$ s to ms time scales. The first generation of LAS sensors deployed in detonation engines used fixed-wavelength telecommunication-grade diode lasers for monitoring temperature, $\mathrm{H}_{2} \mathrm{O}$, fuel, and particulate volume fraction [121]. Later, the use of cesiumabsorption-based sensors for measurements of temperature and pressure at 2000 to $4000 \mathrm{~K}$ and 0.5 to 30 atm [34] and time-of-flight velocimetry [35] were explored using a rapidly scanned VCSEL laser. Mattison et al. [300] developed a scanned-wavelength TDLAS sensor for temperature and $\mathrm{H}_{2} \mathrm{O}$ in a PDE and Klingbeil et al. [104] developed one of the first fibercoupled mid-IR sensors for monitoring JP8 at $3.4 \mu \mathrm{m}$ in a PDE. Recently, IR-LAS sensors for modern detonation systems have shifted towards using hyperspectral and WMS techniques for providing measurements of hydrocarbon-combustion products. This section will focus on several recent manuscripts which have significantly advanced the state of LAS sensors for highly transient, high-pressure combustion flows.

\subsection{Hyperspectral-DA}

Caswell et al. [164, 301] used three time-division multiplexed (TDM) FDML lasers to provide simultaneous measurements of gas temperature, $\mathrm{H}_{2} \mathrm{O}$, pressure, and velocity at $50 \mathrm{kHz}$ in an $\mathrm{H}_{2}$-fueled $\mathrm{PDC}$ at two measurement locations upstream and one downstream of a gas turbine. This sensor used three FDML lasers to perform piecewise continuous-swept-wavelength measurements of the $\mathrm{H}_{2} \mathrm{O}$ absorption spectrum near 1350.5, 1355.85 , and $1362.31 \mathrm{~nm}$ to infer gas properties. Interrogating $\mathrm{H}_{2} \mathrm{O}$ in these spectral windows (identified via 

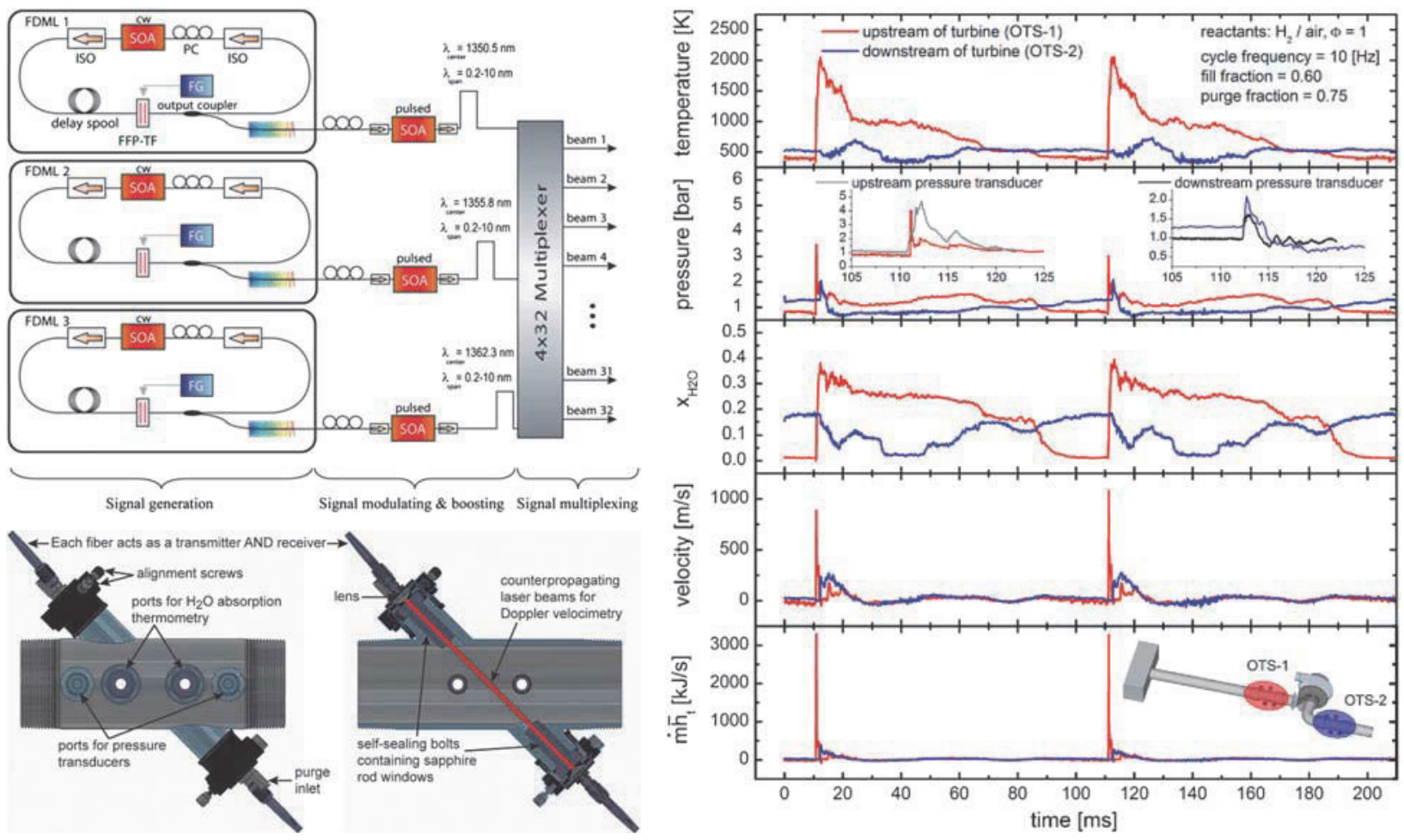

Figure 36: Experimental setup of FDML-based sensor and example of time-resolved temperature, pressure, $\mathrm{H}_{2} \mathrm{O}$, and velocity measurements used to calculate time-resolved enthalpy in an $\mathrm{H}_{2}$-fueled PDC. Figures adapted from [164].

the wavelength-selection algorithm developed by An et al. [161]) enabled near optimal temperature sensitivity. Fig. 36 shows a schematic of the experimental setup used. This sensor also employed novel fiber-optic fixtures that deploy counter-propagating laser bean both angled at $45^{\circ}$ relative to the flow direction to provide velocity measurements in a compact manner.

Fig. 36 shows time-resolved measurements of temperature, pressure, $\mathrm{H}_{2} \mathrm{O}$, velocity and enthalpy for two consecutive PDC cycles. Gas properties were calculated in a manner similar to that of Kranendonk 200 . al. [290] using the HITEMP database [47]. A linear baseline correction was applied to measured and simulated spectra for consistency and instrument broadening was estimated from measurements of $\mathrm{H}_{2} \mathrm{O}$ spectra at 15 torr and $300 \mathrm{~K}$, and was then accounted for 2 im the least-squares fitting routine used to calculate gas properties. Measurements are presented for temperatures and pressures up to $2500 \mathrm{~K}$ and 8 bar without the turbine present, and near $2000 \mathrm{~K}$ and 4 bar with the turbine present. The authors point out thoob measurements could not be acquired until $60 \mu \mathrm{s}$ after the detonation wave passes due to extreme beam steering and pressure broadening. The authors clarify that additional collisional-broadening data is required to improve upon these proof-of-concept pro sure measurements. Regardless, this novel use of multiparameter measurements enabled the calculation of the time-resolved mass flux and enthalpy which provides valuable information for understanding the unsteady performance of the turbine.

\section{2. $W M S$}

Goldenstein et al. [220, 302] and Spearrin et al. [303] recently developed and demonstrated WMS- $2 f / 1 f$ based sensors for temperature, $\mathrm{H}_{2} \mathrm{O}, \mathrm{CO}_{2}$, and $\mathrm{CO}$ in an ethylene-fueled PDC. Measurements were acquired in the combustion chamber and in a choked throat of a converging-diverging nozzle to enable determination of the flow speed, mass flow rate, and enthalpy flow rate from measurements of gas temperature, $\mathrm{H}_{2} \mathrm{O}, \mathrm{CO}_{2}, \mathrm{CO}$ and pressure. Acquiring measurements in a choked throat offers a number of design opportunities, namely, a velocity measurement with sensitivity and accuracy that is nearly independent of pressure (provided that the temperature and species measurements are accurate). This is particularly advantageous when the high pressures (up to $50 \mathrm{~atm}$ ) and pronouned collisional broadening could significantly compromise the precision of a Doppler-shift-based velocity measurement.

All sensors were demonstrated and validated with measurements behind reflected shock waves in shock 

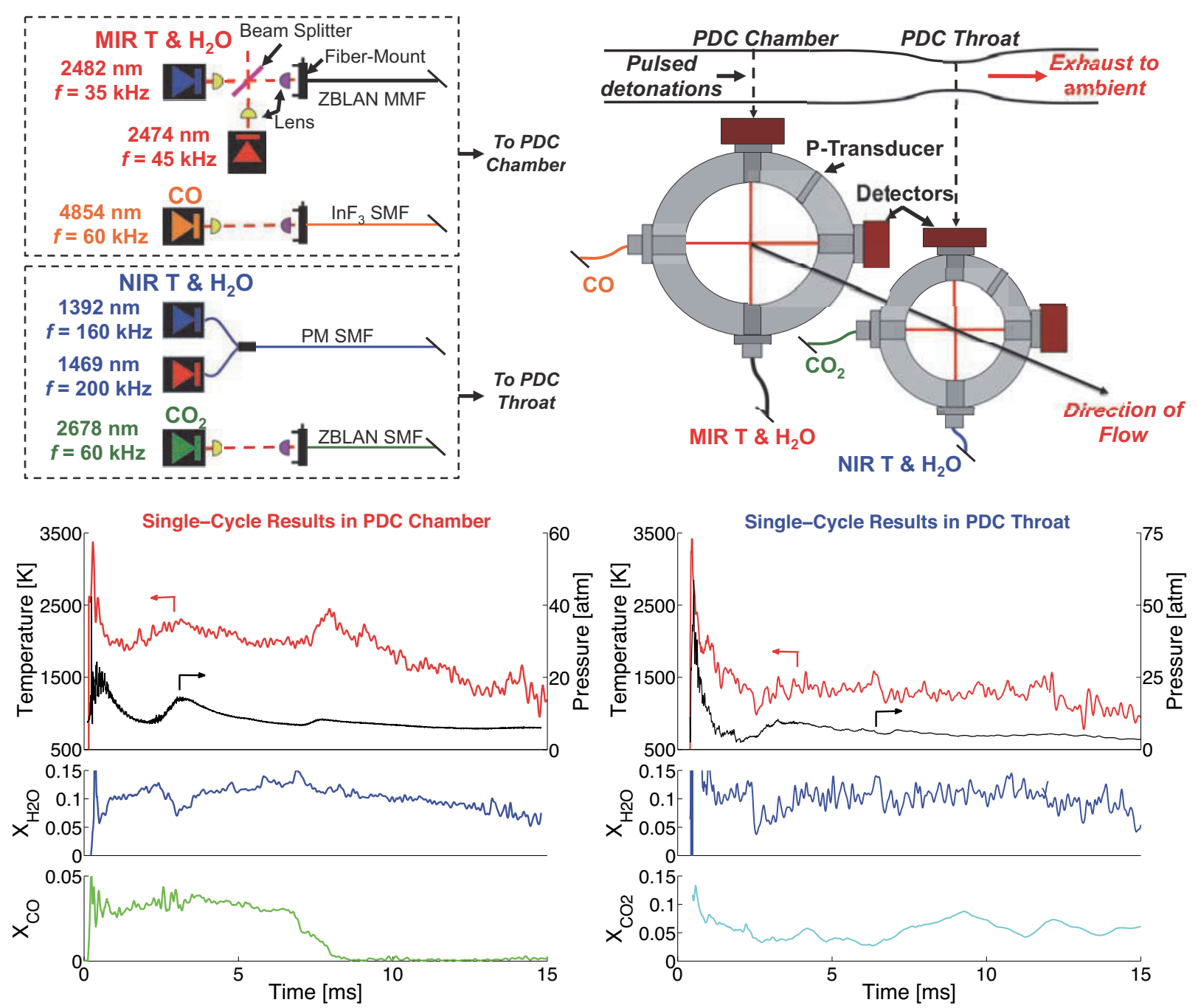

Figure 37: Experimental setup of DFB TDL- and QCL-based WMS- $f f / 1 f$ sensors and example of time-resolved temperature, pressure, $\mathrm{H}_{2} \mathrm{O}, \mathrm{CO}_{2}$, and $\mathrm{CO}$ measurements used to calculate time-resolved enthalpy in an ethylene-fueled PDC. Figures adapted from [46].

tubes at known temperatures and pressures up to 2700 $\mathrm{K}$ and $50 \mathrm{~atm}$. The details regarding the sensor design and validation are documented in $[132,155,97,102]$. DFB diode lasers near 1392 and $1469 \mathrm{~nm}$ were used to measure temperature and $\mathrm{H}_{2} \mathrm{O}$ in the throat with a bandwidth of $10 \mathrm{kHz}$, and DFB TDLs near 2474 on 2482 were used to measure temperature and $\mathrm{H}_{2} \mathrm{O}$ in the combustion chamber with a bandwidth of $9 \mathrm{kHz}$. The DFB TDL near 2678 and a DFB QCL near 4854 $\mathrm{nm}$ were used to measure $\mathrm{CO}_{2}$ and $\mathrm{CO}$ with bandwidths of 2 and $20 \mathrm{kHz}$, respectively. The lasers weres modulated at frequencies from 35 to $200 \mathrm{kHz}$. The near-IR temperature and $\mathrm{H}_{2} \mathrm{O}$ sensor used polarization maintaing SMF, while the mid-IR $\mathrm{H}_{2} \mathrm{O}$ and $\mathrm{CO}_{2}$ sen- sors used ZBLAN fibers, and the CO sensor used an $\mathrm{InF}_{3}$ SMF. All photodetectors were mounted directly to the PDC to facilitate alignment and improve light collection. The wavelengths and modulation depths of all $\mathrm{H}_{2} \mathrm{O}$ lasers were chosen according to the procedure developed by Goldenstein et al. [155] to provide sensitive thermometry and mole fraction sensing from 5 to $50 \mathrm{~atm}$ and 1000 to $3000 \mathrm{~K}$. Free-space fiber coupling and delivery systems were designed for the mid-infrared wavelengths as described by Spearrin et al. $[97,102]$. This involved utilization of newly developed single-mode fluoride glass fibers $(5 \mathrm{~m})$ which significantly mitigated bending losses and multimodal dispersion noise [304] while allowing the sensitive mid- 


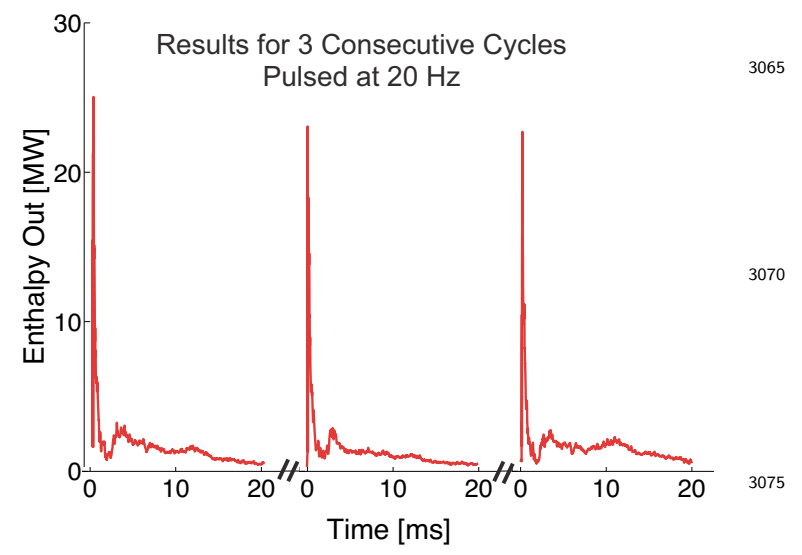

Figure 38: Time-resolved enthalpy flow rate exiting PDC combustor calculated using the LAS measurements shown in Fig. 37 and a choked-flow assumption. Figures adapted from [46].

infrared sources to be located remotely from the com-

Like PDEs, rotating-detonation engines and comı bustors (RDEs, RDCs) represent a promising strategy for achieving the benefits of pressure-gain combustion without moving parts. In an RDE, fuel and air are continuously supplied to an annular combustor and a detonation wave continuously travels around the annulus consuming the air-fuel charge located ahead of the wave. Fig. 39 illustrates this concept via a CFD temperature map reported by Schwer and Kailasanath [305]. This continuous operation has several benefits, including 1) removing the need for a high-frequency repeatable ignition system and 2) enabling much higher operation frequencies (order of $\mathrm{kHz}$ compared to 100 $\mathrm{Hz}$ for $\mathrm{PDE}$ ) which leads to higher thrust/power and a comparatively steady output that is more favorable for integration with gas turbines.

RDEs were first conceptualized in the 1950s, however the complex physics behind their operation delayed the development of functional RDEs until relatively recently. Due to their recent development, only three archival papers have reported the use of LAS sensors for RDEs and all three reported measurements of temperature and $\mathrm{H}_{2} \mathrm{O}[306,138,154]$. These sensors have enabled some insightful observations and conclusions regarding the current state of RDEs and have overcome significant hurdles that complicate the implementation of laser-absorption sensors into RDEs. The remainder of this section will discuss these recent works.

The first LAS sensors were deployed to characterize the exhaust of $\mathrm{H}_{2}$-fueled RDCs [306, 138] and only recently have authors begun developing and deploying sensors for RDC combustors [154]. Regarding sensors for RDC exhaust, McGahan et al. [306] used two VCSELs to measure the absorption spectra of $\mathrm{H}_{2} \mathrm{O}$ from 7435 to $7442 \mathrm{~cm}^{-1}$ and 7465 to 7471 $\mathrm{cm}^{-1}$, thereby enabling calculation of the gas temperature, $\mathrm{H}_{2} \mathrm{O}$ concentration, and velocity using scannedwavelength direct-absorption. The sensor details provided are relatively sparse, however, several conclusions were provided. The TDLAS measurements indicate that the RDE exhaust reaches nominally steady conditions approximately $9 \mathrm{~ms}$ after ignition with a velocity of $364 \pm 110 \mathrm{~m} / \mathrm{s}$ and standard deviations in temperature and $\mathrm{H}_{2} \mathrm{O}$ concentration of $50 \mathrm{~K}$ and 0.08 moles $/ \mathrm{m}^{3}$, respectively. The authors also report how the exhaust gas temperature, velocity and $\mathrm{H}_{2} \mathrm{O}$ concentration varied with equivalence ratio and mass flow rate.

Goldenstein et al. [138] developed and deployed dual two-color LAS sensors using WMS- $2 f / 1 f$ for measurements of temperature and $\mathrm{H}_{2} \mathrm{O}$ at 10 and $50 \mathrm{kHz}$ in the throat of a converging-diverging nozzle attached to an $\mathrm{H}_{2}$-fueled RDC. One pair of diode lasers was used to interrogate $\mathrm{H}_{2} \mathrm{O}$ transitions near 2551 and $2482 \mathrm{~nm}$ with $E$ " of 704 and $2660 \mathrm{~cm}^{-1}$, respectively, and another pair of diode lasers was used to interrogate $\mathrm{H}_{2} \mathrm{O}$ transitions near 1392 and $1469 \mathrm{~nm}$ with $E$ " of 1045 and $3291 \mathrm{~cm}^{-1}$, respectively. This was done to quantify the 
a)

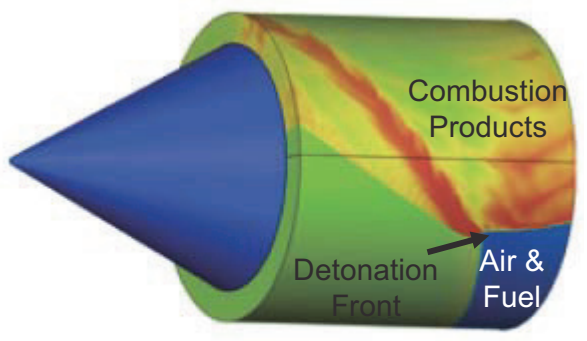

b)

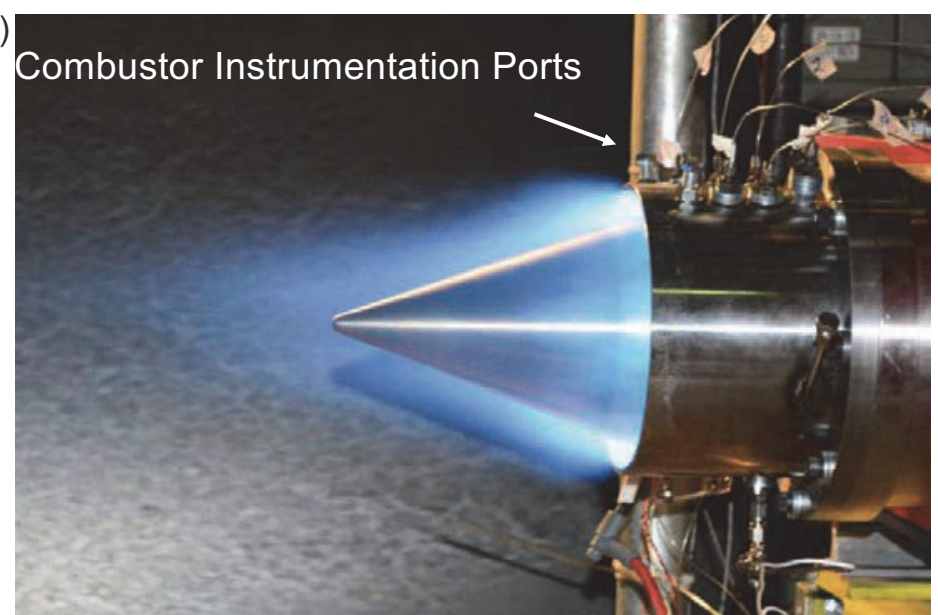

Figure 39: Simulated temperature distribution in an RDC (a, figure adapted from [305]) and $\mathrm{H}_{2}$-fueled RDC outfitted with single-ended LAS sensor (b, figure adapted from [154]).

thermal uniformity of the exhaust using a method similar to that of Seitzman and Scully [174]. Measurements to 1 (achieved by varying the mass flow rate of air with fuel flow rate fixed). In all cases, the temperature measurements acquired at $50 \mathrm{kHz}$ clearly revealed the oscillations near $\pm 250 \mathrm{~K}$ at the characteristic frequency of the detonation wave $(\approx 3.25 \mathrm{kHz})$. The difference between the "hot" and "cold" temperatures measured by the near-IR and mid-IR lasers, respectively, was found to increase from 2 to $8 \%$ as the air flow rate increased from 1.2 to $1.5 \mathrm{~kg} / \mathrm{s}$ indicating a pronounced correlacation of the exhaust. In addition, the $\mathrm{H}_{2} \mathrm{O}$ production was found to decrease only slightly with increasing air flow rate (i.e., decreasing equivalence ratio) suggesting that air-fuel mixing was the primary factor leading to

Most recently, Rein et al. [154] presented the first measurements of temperature and $\mathrm{H}_{2} \mathrm{O}$ in the annular combustor using a single-ended fiber-coupled sensor. The authors used a MEMS VCSEL to measure at $100 \mathrm{kHz}$ and yield temperature and $\mathrm{H}_{2} \mathrm{O}$ concentration. A fiber-bundle housing a single-mode pitch fiber and a multi-mode catch fiber (see Fig. 22) was used to pitch laser light into the combustor and collect the 3145 light backscattered off the RDE centerbody. The anlls nular gap in the $\mathrm{H}_{2}$-fueled $\mathrm{RDE}$ was $0.76 \mathrm{~cm}$ yielding an effective path length of $1.52 \mathrm{~cm}$ and measurements were acquired 25.4 and $50.8 \mathrm{~mm}$ downstream of the fuel plenum. The authors found a strong correlation between the gas temperature and air-mass flow ratre which they primarily attributed to the location of the measurement with respect to that of the detonation

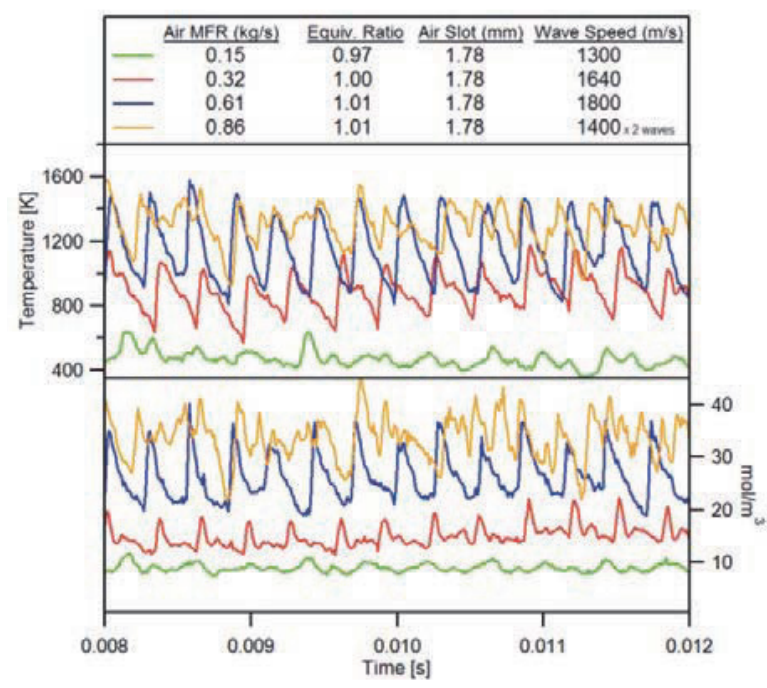

Figure 40: Example of time-resolved gas temperature and $\mathrm{H}_{2} \mathrm{O}$ acquired via a single-ended LAS sensor (see Figure 22) in an RDC combustor. Reprinted from [154] with permission from AIAA.

wave (it was previously shown that the axial-depth of the detonation wave exhibits a strong correlation with the air mass flow rate [307]). Fig. 40 shows that the nominal gas temperature varied from near $500 \mathrm{~K}$ up to $1200 \mathrm{~K}$ as the air mass flow rate increased from 0.15 to $0.61 \mathrm{~kg} / \mathrm{s}$ with a fixed equivalence ratio near 1. In addition, the authors found that the gas temperature measured at the downstream location was significantly colder than that of the upstream location located within the detonation wave. 


\section{Conclusions and Outlook}

Over the last 15 years, great progress has been made

3165 combustors, and even in-flight experiments onboard a scramjet. This progress has proven that IR-LAS sensors can be applied to virtually any combustion system of interest.

Despite this progress, extensive research remains needed. Spectroscopic databases for molecular spectra at high-temperatures and -pressures continue to suffer from unsatisfactory accuracy, primarily due to errors and complexities associated with calculating collisional-broadening coefficients. Furthermore, these databases are applicable to a relatively small number of species of interest to combustion (namely, $\mathrm{CO}, \mathrm{CO}_{2}$, $\mathrm{H}_{2} \mathrm{O}, \mathrm{NO}, \mathrm{OH}$ ). As a result, research to improve the accuracy and breadth of spectroscopic databases rele-

3210 vant to combustion is needed, particularly to improve sensor accuracy and assist the design of sensors for high pressures.

Improvements in mid-infrared hardware and optical materials are also needed. While useful, the power, 3215 stability, tuning ability, and life-time of mid-infrared lasers is frequently inferior to their near-IR analogues.
This currently limits the accuracy and precision of midinfrared sensors below what is theoretically obtainable (e.g., due to their increased absorption strength) and complicates the integration of mid-infrared sensors into practical combustion systems outside the laboratory. Improvements in mid-infrared fiber and optical materials are also needed. Mid-IR fibers suffer from significantly larger bend losses and transmission losses which complicates or precludes remote placement of lasers. Materials that can tolerate high temperatures, pressures, and combustion gases with high transmission levels beyond $5 \mu \mathrm{m}$ are also needed. This currently represents a major obstacle to improved detection of several species important to combustion systems (e.g., $\mathrm{NO}, \mathrm{NH}_{3}$, hydrocarbons). Extending recently developed single-ended LAS packages to the mid-infrared is also needed.

Lastly, sensors for multi-species measurements and measurements at high pressures remain research fronts. While some promising work has been conducted, the potential of IR-LAS to expand sensing capabilities in these areas has yet to be realized. Prospects for improvement include the use of hyperspectral lightsources which can access multiple absorption bands and WMS techniques incorporating novel modulation techniques and frequency-multiplexing of multiple narrowband light sources.

We expect that the role of IR-LAS in combustion will continue to grow as researchers continue to improve and expand the capabilities of IR-LAS and improved light sources and hardware are developed. While some techniques are only in their infancy, others have matured into robust sensors capable of characterizing a wide range of practical combustion systems. As a result, we now embark on a time where the potential to not only improve our understanding of, but also control combustion devices has never been greater.

\section{Acknowledgements}

The authors thank their many colleagues worldwide for allowing their work to be discussed and shown here. Further, the authors would like to thank the many present and past students of the Hanson Group at Stanford University who have helped support many of the LAS-sensing efforts discussed here. Lastly, Ron Hanson expresses his deepest gratitude to the AFOSR, ARO, DoE, and ONR for supporting the research and application of LAS sensors over the last 40 years.

\section{References}

[1] L. S. Rothman, I. Gordon, Y. Babikov, A. Barbe, D. Chris Benner, P. Bernath, M. Birk, L. Bizzocchi, V. Boudon, L. Brown, A. Campargue, K. Chance, E. Cohen, L. Coudert, V. Devi, B. Drouin, A. Fayt, J.-M. Flaud, R. R. Gamache, 
J. Harrison, J.-M. Hartmann, C. Hill, J. Hodges, D. Jacquemart, A. Jolly, J. Lamouroux, R. Le Roy, G. Li, D. Long, O. Lyulin, C. Mackieg S. Massie, S. Mikhailenko, H. Müller, O. Naumenko, A. Nikitin, J. Orphal, V. Perevalov, A. Perrin, E. Polovtseva, C. Richard, M. Smith, E. Starikova, K. Sung, S. Tashkun, J. Tennyson, G. Toon, V. G. Tyuterev, G. Wagner, The HITRAN2012 molecular spectroscopic database, Journal of Quantitative Spectroscopy and Radi325 tive Transfer 130 (2013) 4-50.

[2] J. Wolfrum, Lasers in combustion: from basic theory to practical devices, Proceedings of the Combustion Institute (1998) 1-41.

[3] R. K. Hanson, Applications of quantitative laser sensors to kinetics, propulsion and practical energy systems, Proceedings of the Combustion Institute 33 (2011) 1-40.

[4] M. G. Allen, Diode laser absorption sensors for gas-dynamic and combustion flows., Measurement science \& technology 9 (1998) 545-62.

[5] P. Werle, A review of recent advances in semiconductor laser based gas monitors, Spectrochimica Acta Part A: Molecular and Biomolecular Spectroscopy 54 (1998) 197-236.

[6] M. Lackner, Tunable diode laser absorption spectroscopy (TDLAS) in the process industries-a review 23 (2007).

[7] C. Schulz, A. Dreizler, V. Ebert, J. Wolfrum, $39_{5}$ Combustion Diagnostics, Springer Handbook of Experimental Fluid Mechanics (2007) 1241-1315.

[8] M. Bolshov, Y. Kuritsyn, Y. Romanovskii, Tunable diode laser spectroscopy as a technique for combustion diagnostics, Spectrochimica Acta Part B: Atomic Spectroscopy 106 (2015) 45-66 $6_{350}$

[9] A. C. Eckbreth, Laser diagnostics for combustion temperatures and species, 2nd ed., Taylor and Francis, London, 1996.

[10] K. Kohse-Hoinghaus, J. B. Jeffries, Applied Combustion Diagnostics, Taylor and Francis New York, 2002.

[11] R. K. Hanson, R. M. Spearrin, C. S. Goldenstein, Spectroscopy and Optical Diagnostics for Gases, Springer International Publishing Switzerland, 2016.

[12] K. G. P. Sulzmann, J. E. L. Lowder, S. S. Penner, Estimates of possible detection limits for combustion intermediates and products with line- center absorption and derivative spectroscopy using tunable lasers, Combustion and Flame 20 (1973) 177-191.

[13] R. K. Hanson, P. A. Kuntz, C. H. Kruger, Highresolution spectroscopy of combustion gases using a tunable ir diode laser, Applied Optics 16 (1977) 2045-2048.

[14] R. K. Hanson, Shock tube spectroscopy: advanced instrumentation with a tunable diode laser, Applied Optics 16 (1977) 1479-1481.

[15] R. K. Hanson, P. K. Falcone, Temperature measurement technique for high-temperature gases using a tunable diode laser., Applied Optics 17 (1978) 2477-2480.

[16] J. Reid, J. Shewchun, B. K. Garside, E. A. Ballik, High sensitivity pollution detection employing tunable diode lasers, Applied Optics 17 (1978) 300-307.

[17] J. Reid, B. K. Garside, J. Shewchun, M. ElSherbiny, E. a. Ballik, High sensitivity point monitoring of atmospheric gases employing tunable diode lasers., Applied optics 17 (1978) 180610.

[18] J. Reid, M. El-Sherbiny, B. K. Garside, E. A. Ballik, Sensitivity limits of a tunable diode laser spectrometer, with application to the detection of $\mathrm{NO}_{2}$ at the 100-ppt level, Applied Optics 19 (1980) 3349-3354.

[19] J. Reid, D. Labrie, Second-Harmonic Detection with Tunable Diode Lasers - Comparison of Experiment and Theory*, Applied Physics B 26 (1981) 203-210.

[20] G. C. Bjorklund, Frequency-modulation spectroscopy: a new method for measuring weak absorptions and dispersions, Optics Letters 5 (1980) 15-17.

[21] D. T. Cassidy, J. Reid, Harmonic detection with tunable diode lasers-two-tone modulation*, Applied Physics B 229 (1982) 279-285.

[22] G. C. Bjorklund, M. D. Levenson, W. Lenth, C. Ortiz, Frequency modulation (FM) spectroscopy, Applied Physics B 32 (1983) 145-152.

[23] W. Lenth, High frequency heterodyne spectroscopy with current-modulated diode lasers, IEEE Journal of Quantum Electronics 20 (1984) 1045-1050. 
[24] D. T. Cassidy, Trace gas detection using $1.3 \mu \mathrm{m}$ InGaAsP diode laser transmitter modules, Applied Optics 27 (1988) 610-614.

[25] L. C. Philippe, R. K. Hanson, Laser-absorptiøns mass flux sensor for high-speed airflows, Optics Letters 16 (1991) 2002-2004.

[26] A. Sappey, P. Mccormick, P. Masterson, Q. Zhao, L. Sutherland, I. Smith, P. Vanhoudt, J. Hannam, D. Owenby, Development of a fliglate worthy TDLAS-based oxygen sensor for HIFiRE1, in: 45th AIAA/ASME/SAE/ASEE Joint Propulsion Conference \& Exhibit, August, 2009, pp. AIAA 2009-4971.

[27] S.-J. Chen, M. Paige, J. Silver, S. Williamss, T. Barhorst, Laser-based mass flow rate sensor onboard HFiRE Flight 1, in: 45th AIAA/ASME/SAE/ASEE Joint Propulsion Conference \& Exhibit, August 2009, 2009, pp. AIAA 2009-5065.

[28] M. Brown, T. Barhorst, Post-flight analysis of the diode-laser-based mass capture experiment onboard HIFiRE flight 1, in: 17th AIAA International Space Planes and Hypersonic Systems and Technologies Conference, April, 2011, pp. AIAust 2011-2359.

[29] M. P. Arroyo, R. K. Hanson, Absorption measurements of water-vapor concentration, temperature, and line-shape parameters using a tunable InGaAsP diode laser, Applied Optics 32 (199939 6104-6116.

[30] D. S. Baer, R. K. Hanson, M. E. Newfield, N. K. Gopaul, Multiplexed diode-laser sensor system for simultaneous $\mathrm{H}_{2} \mathrm{O}, \mathrm{O}_{2}$, and temperature measurements, Optics Letters 19 (1994) 1900-1902.

[31] D. S. Baer, V. Nagali, E. R. Furlong, R. K. Hanson, M. E. Newfield, Scanned- and fixedwavelength absorption diagnostics for combustion measurements using multiplexed dioded lasers, AIAA Journal 34 (1996) 489-493.

[32] E. R. Furlong, R. M. Mihalcea, M. E. Webber, D. S. Baer, R. K. Hanson, Diode-laser sensors for real-time control of pulsed combustion systems, AIAA Journal 37 (1999) 732-737.

[33] J. Wang, S. T. Sanders, J. B. Jeffries, R. K. Hanson, Oxygen measurements at high pressures with vertical cavity surface-emitting lasers, Applied Physics B 72 (2001) 865-872. 3460
[34] S. T. Sanders, D. W. Mattison, L. Ma, J. B. Jeffries, R. K. Hanson, Wavelength-agile diodelaser sensing strategies for monitoring gas properties in optically harsh flows: application in cesium-seeded pulse detonation., Optics Express 10 (2002) 505-14.

[35] S. T. Sanders, D. W. Mattison, J. B. Jeffries, R. K. Hanson, Time-of-flight diode-laser velocimeter using a locally seeded atomic absorber: Application in a pulse detonation engine, Shock Waves 12 (2003) 435-441.

[36] L. A. Kranendonk, J. W. Walewski, T. Kim, S. T. Sanders, Wavelength-agile sensor applied for HCCI engine measurements, Proceedings of the Combustion Institute 30 (2005) 1619-1627.

[37] L. A. Kranendonk, X. An, A. W. Caswell, R. E. Herold, S. T. Sanders, R. Huber, J. G. Fujimoto, Y. Okura, Y. Urata, High speed engine gas thermometry by Fourier-domain mode-locked laser absorption spectroscopy., Optics Express 15 (2007) 15115-28.

[38] L. A. Kranendonk, A. W. Caswell, C. L. Hagen, C. T. Neuroth, D. T. Shouse, J. R. Gord, S. T. Sanders, Temperature measurements in a gas-turbine-combustor sector rig using sweptwavelength absorption spectroscopy, Journal of Propulsion and Power 25 (2009) 859-863.

[39] T. Fernholz, H. Teichert, V. Ebert, Digital, phase-sensitive detection for in situ diode-laser spectroscopy under rapidly changing transmission conditions, Applied Physics B 75 (2002) 229-236.

[40] H. Li, G. B. Rieker, X. Liu, J. B. Jeffries, R. K. Hanson, Extension of wavelength-modulation spectroscopy to large modulation depth for diode laser absorption measurements in high-pressure gases, Applied Optics 45 (2006) 1052-1061.

[41] G. B. Rieker, J. B. Jeffries, R. K. Hanson, Calibration-free wavelength-modulation spectroscopy for measurements of gas temperature and concentration in harsh environments., Applied Optics 48 (2009) 5546-60.

[42] G. B. Rieker, H. Li, X. Liu, J. T. C. Liu, J. B. Jeffries, R. K. Hanson, M. G. Allen, S. D. Wehe, P. Mulhall, H. Kindle, A. Kakuho, K. R. Sholes, T. Matsuura, S. Takatani, Rapid measurements of temperature and $\mathrm{H}_{2} \mathrm{O}$ concentration in IC engines with a spark plug-mounted diode laser sensor, Proceedings of the Combustion Institute 31 (2007) 3041-3049. 
[43] G. Rieker, J. Jeffries, R. Hanson, T. Mathur, M. Gruber, C. Carter, Diode laser-based detection of combustor instabilities with application to a scramjet engine, Proceedings of the Combustion Institute 32 (2009) 831-838.

[44] A. E. Klingbeil, J. B. Jeffries, R. K. Hanson, Temperature- and composition-dependent midinfrared absorption spectrum of gas-phase gasoline: Model and measurements, Fuel 87 (2008) 3600-3609.

[45] P. L. Varghese, Tunable infrared diode laser measurements of spectral parameters of carbon monoxide and hydrogen cyanide, Ph.D. thesis, Stanford University, 1983.

[46] C. S. Goldenstein, R. K. Hanson, Diode-laser measurements of linestrength and temperature-5 dependent lineshape parameters for $\mathrm{H}_{2} \mathrm{O}$ transitions near $1.4 \mu \mathrm{m}$ using Voigt, Rautian, Galatry, and speed-dependent Voigt profiles, Journal of Quantitative Spectroscopy and Radiative Transfer 152 (2015) 127-139.

[47] L. S. Rothman, I. Gordon, R. Barber, H. Dothe, R. R. Gamache, A. Goldman, V. Perevalov, S. Tashkun, J. Tennyson, HITEMP, the hightemperature molecular spectroscopic database, Journal of Quantitative Spectroscopy and Radiative Transfer 111 (2010) 2139-2150.

[48] J. Humlicek, Optimized computation of the Voigt and complex probability functions, Journal of Quantitative Spectroscopy and Radiative Transfer 27 (1982) 437-444.

[49] M. Kuntz, A new implementation of the Humlicek algorithm for the calculation of the Voigt profile function, Journal of Quantitative Spectroscopy and Radiative Transfer 51 (1997) 819824.

[50] W. Ruyten, Comment on A new implementati3545 of the Humlicek algorithm for the calculation of the Voigt profile function by M. Kuntz [JQSRT 57(6) (1997) 819824], Journal of Quantitative Spectroscopy and Radiative Transfer 86 (2004) 231-233.

[51] A. McLean, C. Mitchell, D. Swanston, Impł⿷50 mentation of an efficient analytical approximation to the Voigt function for photoemission lineshape analysis, Journal of Electron Spectroscopy and Related Phenomena 69 (1994) 125-132.

[52] R. Dicke, The effect of collisions upon thase Doppler width of spectral lines, Physical Review 89 (1953) 472-473.
[53] S. Rautian, I. Sobel'man, The effect of collisions on the Doppler broadening of spectral lines, Sov Phys Uspekhi 9 (1967) 701-716.

[54] L. Galatry, Simultaneous effect of Doppler and foreign gas broadening on spectral lines, Physical Review 122 (1961) 1218-1223.

[55] N. Ngo, D. Lisak, H. Tran, J.-M. Hartmann, An isolated line-shape model to go beyond the Voigt profile in spectroscopic databases and radiative transfer codes, Journal of Quantitative Spectroscopy and Radiative Transfer 129 (2013) 89100.

[56] J. Ward, J. Cooper, E. W. Smith, Correlation effects in the theory of combined Doppler and pressure broadening-I. Classical theory*, Journal of Quantitative Spectroscopy and Radiative Transfer 14 (1974) 555-590.

[57] F. Rohart, H. Mader, H.-W. Nicolaisen, Speed dependence of rotational relaxation induced by foreign gas collisions: Studies on $\mathrm{CH}_{3} \mathrm{~F}$ by millimeter wave coherent transients, The Journal of Chemical Physics 101 (1994) 6475.

[58] C. D. Boone, K. A. Walker, P. F. Bernath, Speeddependent Voigt profile for water vapor in infrared remote sensing applications, Journal of Quantitative Spectroscopy and Radiative Transfer 105 (2007) 525-532.

[59] J.-M. Hartmann, M. Y. Perrin, Q. Ma, R. H. Tipping, The infrared continuum of pure water vapor: calculations and high-temperature measurements, Journal of Quantitative Spectroscopy and Radiative Transfer 49 (1993) 675-691.

[60] S. A. Clough, F. X. Kneizys, R. W. Davies, Line shape and the water vapor continuum, Atmospheric Research 23 (1989) 229-241.

[61] G. B. Rieker, X. Liu, H. Li, J. B. Jeffries, R. K. Hanson, Measurements of near-IR water vapor absorption at high pressure and temperature, Applied Physics B 87 (2006) 169-178.

[62] J.-M. Hartmann, C. Boulet, D. Robert, Collisional Effects on Molecular Spectra, Elsevier Inc., 2008.

[63] A. Farooq, J. B. Jeffries, R. K. Hanson, Highpressure measurements of $\mathrm{CO}_{2}$ absorption near $2.7 \mu \mathrm{m}$ : Line mixing and finite duration collision effects, Journal of Quantitative Spectroscopy and Radiative Transfer 111 (2010) 949-960. 
[64] V. Nagali, Diode laser study of high-pressure water-vapor spectroscopy, Ph.D. thesis, Stanford University, 1998.

[65] C. Christiansen, T Stolberg-Rohr, A. Fate ${ }^{3610}$ S. Clausen, High temperature and high pressure gas cell for quantitative spectroscopic measurements, Journal of Quantitative Spectroscopy and Radiative Transfer 169 (2016) 96-103.

[66] R. S. Eng, A. R. Calawa, T. C. Harman, P. L. Kelley, A. Javan, Collisional narrowing of infrared water-vapor transitions, Applied Physics Letters 21 (1972) 303-305. Diode laser measurements of linestrength and

temperature-dependent lineshape parameters of $\mathrm{H}_{2} \mathrm{O}-, \mathrm{CO}_{2^{-}}$, and $\mathrm{N}_{2}$-perturbed $\mathrm{H}_{2} \mathrm{O}$ transitions near 2474 and $2482 \mathrm{~nm}$, Journal of Quantitative Spectroscopy and Radiative Transfer 130 (2013) 100-111.

[75] V. Nagali, R. K. Hanson, Design of a diode-laser sensor to monitor water vapor in high-pressure combustion gases, Applied Optics 36 (1997) 9518-9527.

[76] M. Perrin, J. Hartmann, Temperature-dependent measurements and modeling of absorption by $\mathrm{CO}_{2}-\mathrm{N}_{2}$ mixtures in the far line-wings of the 4.3 $\mu \mathrm{m} \mathrm{CO} 2$ band, Journal of Quantitative Spectroscopy and Radiative Transfer 42 (1989) 311317.

[77] G. B. Rieker, J. B. Jeffries, R. K. Hanson, Measurements of high-pressure $\mathrm{CO}_{2}$ absorption near $2.0 \mu \mathrm{m}$ and implications on tunable diode laser sensor design, Applied Physics B 94 (2008) 5163.

[78] S. Stefani, G. Piccioni, M. Snels, D. Grassi, A. Adriani, Experimental $\mathrm{CO}_{2}$ absorption coefficients at high pressure and high temperature, Journal of Quantitative Spectroscopy and Radiative Transfer 117 (2013) 21-28.

[79] F. Niro, K. Jucks, J.-M. Hartmann, Spectra calculations in central and wing regions of IR bands. IV: software and database for the computation of atmospheric spectra, Journal of Quantitative Spectroscopy and Radiative Transfer 95 (2005) 469-481.

[80] N. N. Filippov, R. E. Asfin, T. N. Sinyakova, I. M. Grigoriev, T. M. Petrova, A. M. Solodov, A. A. Solodov, J. V. Buldyreva, Experimental and theoretical studies of $\mathrm{CO}_{2}$ spectra for planetary atmosphere modelling: region 600$9650 \mathrm{~cm}^{-1}$ and pressures up to $60 \mathrm{~atm}$., Physical chemistry chemical physics 15 (2013) 1382613834 .

[81] H. Tran, C. Boulet, S. Stefani, M. Snels, G. Piccioni, Measurements and modelling of high pressure pure $\mathrm{CO}_{2}$ spectra from 750 to $8500 \mathrm{~cm}^{-1}$. Icentral and wing regions of the allowed vibrational bands, Journal of Quantitative Spectroscopy and Radiative Transfer 112 (2011) 925936.

[82] M. Alberti, R. Weber, M. Mancini, Re-creating Hottel's emissivity charts for carbon dioxide and extending them to 40 bar pressure using HITEMP-2010 data base, Combustion and Flame 162 (2015) 597-612. 
[83] J. Lamouroux, L. Régalia, X. Thomas, J. Vander Auwera, R. Gamache, J.-M. Hartmann, CQn line-mixing database and software update and its tests in the $2.1 \mu \mathrm{m}$ and $4.3 \mu \mathrm{m}$ regions, Journal of Quantitative Spectroscopy and Radiative Transfer 151 (2015) 88-96.

[84] R. Sur, R. M. Spearrin, W. Y. Peng, C. ${ }_{355}$ Strand, J. B. Jeffries, G. M. Enns, R. K. Hanson, Line intensities and temperature-dependent line broadening coefficients of Q-branch transitions in the $\nu_{2}$ band of ammonia near $10.4 \mu \mathrm{m}$, Journal of Quantitative Spectroscopy and Radiative Transfer 175 (2016) 90-99.

[85] N. Jacquinet-Husson, N. Scott, A. Chédin, L. Crépeau, R. Armante, V. Capelle, J. Orphal, A. Coustenis, C. Boonne, N. Poulet-Crovisier, A. Barbe, M. Birk, L. Brown, C. Camy-

[86] S. Tashkun, V. Perevalov, J.-L. Teffo, A. Bykov, N. Lavrentieva, CDSD-1000, the hightemperature carbon dioxide spectroscopic databank, Journal of Quantitative Spectroscopy and Radiative Transfer 82 (2003) 165-196.

[87] S. Tashkun, V. Perevalov, CDSD-4000: Highresolution, high-temperature carbon dioxide spectroscopic databank, Journal of Quantitative Spectroscopy and Radiative Transfer 112 (2011) 1403-1410.

[88] R. J. Barber, J. Tennyson, G. J. Harris, R. N. Tolchenov, A high-accuracy computed water line list, Monthly Notices of the Royal Astronomical Society 368 (2006) 1087-1094.
[90] P. Chu, F. Guenther, G. Rhoderick, W. Lafferty, The NIST quantitative infrared database, Journal of Research of the National Institute of Standards and Technology 104 (1999).

[91] R. A. Toth, Measurements of positions, strengths and self-broadened widths of $\mathrm{H}_{2} \mathrm{O}$ from 2900 to $8000 \mathrm{~cm}^{-1}$ : line strength analysis of the $2 \mathrm{nd}$ triad bands, Journal of Quantitative Spectroscopy and Radiative Transfer 94 (2005) 51-107.

[92] R. A. Toth, L. Brown, M. Smith, V. Malathy Devi, D. Chris Benner, M. Dulick, Airbroadening of $\mathrm{H}_{2} \mathrm{O}$ as a function of temperature: 696-2163 $\mathrm{cm}^{-1}$, Journal of Quantitative Spectroscopy and Radiative Transfer 101 (2006) 339366.

[93] X. Liu, J. B. Jeffries, R. K. Hanson, Measurements of spectral parameters of water-vapour transitions near 1388 and $1345 \mathrm{~nm}$ for accurate simulation of high-pressure absorption spectra, Measurement Science and Technology 18 (2007) 1185-1194.

[94] X. Liu, X. Zhou, J. B. Jeffries, R. K. Hanson, Experimental study of $\mathrm{H}_{2} \mathrm{O}$ spectroscopic parameters in the near-IR $\left(6940-7440 \mathrm{~cm}^{-1}\right)$ for gas sensing applications at elevated temperature, Journal of Quantitative Spectroscopy and Radiative Transfer 103 (2007) 565-577.

[95] A. Pogány, A. Klein, V. Ebert, Measurement of water vapor line strengths in the 1.4-2.7 $\mu \mathrm{m}$ range by tunable diode laser absorption spectroscopy, Journal of Quantitative Spectroscopy and Radiative Transfer 165 (2015) 108-122.

[89] S. W. Sharp, T. Johnson, R. Sams, P. Chus G. Rhoderick, P. Johnson, Gas-phase databases for quantitative infrared spectroscopy, Applied Spectroscopy 58 (2004) 1452-1461.
[96] S. T. Melin, S. T. Sanders, Gas cell based on optical contacting for fundamental spectroscopy studies with initial reference absorption spectrum of $\mathrm{H}_{2} \mathrm{O}$ vapor at $1723 \mathrm{~K}$ and 0.0235 bar, Journal of Quantitative Spectroscopy and Radiative Transfer 180 (2016) 184-191.

[97] R. M. Spearrin, C. S. Goldenstein, J. B. Jeffries, R. K. Hanson, Fiber-coupled $2.7 \mu \mathrm{m}$ laser absorption sensor for $\mathrm{CO}_{2}$ in harsh combustion environments, Measurement Science and Technology 24 (2013) 055107.

[98] A. Farooq, J. Jeffries, R. Hanson, $\mathrm{CO}_{2}$ concentration and temperature sensor for combustion gases using diode-laser absorption near $2.7 \mu \mathrm{m}$, Applied Physics B 90 (2008) 619-628.

[99] R. M. Spearrin, W. Ren, J. B. Jeffries, R. K. Hanson, Multi-band infrared $\mathrm{CO}_{2}$ absorption sen- 
sor for sensitive temperature and species measurements in high-temperature gases, Applied Physics B 116 (2014) 855-865.

[100] P. J. Medvecz, K. M. Nichols, Experimental deo termination of line strengths for selected carbon monoxide and carbon dioxide absorption lines at temperatures between 295 and $1250 \mathrm{~K}$, Applied Spectroscopy 48 (1994) 1442-1450.

[101] J. Vanderover, W. Wang, M. A. Oehlschlaeger, ,3A carbon monoxide and thermometry sensor based on mid-IR quantum-cascade laser wavelengthmodulation absorption spectroscopy, Applied Physics B 103 (2011) 959-966.

[102] R. M. Spearrin, C. S. Goldenstein, J. B. Jeffriæs R. K. Hanson, Quantum cascade laser absorption sensor for carbon monoxide in high-pressure gases using wavelength modulation spectroscopy, Applied Optics 53 (2014) 1938-1946.

[103] R. Sur, K. Sun, J. B. Jeffries, R. K. Hraks son, Multi-species laser absorption sensors for in situ monitoring of syngas composition, Applied Physics B 115 (2013) 9-24.

[104] A. E. Klingbeil, J. B. Jeffries, R. K. Hanson, Design of a fiber-coupled mid-infrared fuel sensese for pulse detonation engines, AIAA Journal 45 (2007) $772-778$.

[105] M. Sajid, T. Javed, A. Farooq, High-temperature measurements of methane and acetylene using quantum cascade laser absorption near $8 \mu$ m Journal of Quantitative Spectroscopy and Radiative Transfer 155 (2015) 66-74.

[106] R. Sur, S. Wang, K. Sun, D. F. Davidson, J. B. Jeffries, R. K. Hanson, High-sensitivity interference-free diagnostic for measurement 3 methane in shock tubes, Journal of Quantitative Spectroscopy and Radiative Transfer 156 (2015) 80-87.

[107] W. Ren, D. F. Davidson, R. K. Hanson, IR laser absorption diagnostic for $\mathrm{C}_{2} \mathrm{H}_{4}$ in shock tube bisis netics studies, International Journal of Chemical Kinetics 44 (2012) 423-432.

[108] E.-t. Es-sebbar, M. Alrefae, A. Farooq, Infrared cross-sections and integrated band intensities of propylene: Temperature-dependent sturbo ies, Journal of Quantitative Spectroscopy and Radiative Transfer 133 (2014) 559-569.

[109] R. S. M. Chrystie, E. F. Nasir, A. Farooq, Propene concentration sensing for combustion gases using quantum-cascade laser absorptiæm near $11 \mu \mathrm{m}$, Applied Physics B 120 (2015) 317327.

[110] R. Spearrin, S. Li, D. Davidson, J. Jeffries, R. Hanson, High-temperature iso-butene absorption diagnostic for shock tube kinetics using a pulsed quantum cascade laser near $11.3 \mu \mathrm{m}$, Proceedings of the Combustion Institute 35 (2015) 3645-3651.

[111] R. M. Spearrin, I. A. Schultz, J. B. Jeffries, R. K. Hanson, Laser absorption of nitric oxide for thermometry in high-enthalpy air, Measurement Science and Technology 25 (2014) 125103.

[112] R. Sur, W. Y. Peng, C. L. Strand, R. M. Spearrin, J. B. Jeffries, R. K. Hanson, Mid-infrared laser absorption spectroscopy of $\mathrm{NO}_{2}$ at elevated temperatuers, Journal of Quantitative Spectroscopy and Radiative Transfer (2016).

[113] S. Schilt, Impact of water vapor on $1.51 \mu \mathrm{m}$ ammonia absorption features used in trace gas sensing applications, Applied Physics B 100 (2010) 349-359.

[114] K. Owen, E.-t. Es-sebbar, A. Farooq, Measurements of $\mathrm{NH}_{3}$ linestrengths and collisional broadening coefficients in $\mathrm{N}_{2}, \mathrm{O}_{2}, \mathrm{CO}_{2}$, and $\mathrm{H}_{2} \mathrm{O}$ near $1103.46 \mathrm{~cm}^{-1}$, Journal of Quantitative Spectroscopy and Radiative Transfer 121 (2013) 5668.

[115] M. Dhib, J.-P. Bouanich, H. Aroui, A. PicardBersellini, Analysis of $\mathrm{N}_{2}, \mathrm{O}_{2}, \mathrm{CO}_{2}$, and air broadening of infrared spectral lines in the $\nu_{4}$ band of $\mathrm{NH}_{3}$, Journal of Quantitative Spectroscopy and Radiative Transfer 68 (2001) 163178.

[116] A. E. Klingbeil, J. B. Jeffries, R. K. Hanson, Temperature-dependent mid-IR absorption spectra of gaseous hydrocarbons, Journal of Quantitative Spectroscopy and Radiative Transfer 107 (2007) 407-420.

[117] E. T. Es-Sebbar, Y. Benilan, A. Farooq, Temperature-dependent absorption cross-section measurements of 1-butene $\left(1-\mathrm{C}_{4} \mathrm{H}_{8}\right)$ in VUV and IR, Journal of Quantitative Spectroscopy and Radiative Transfer 115 (2013) 1-12.

[118] E. Es-sebbar, A. Jolly, Y. Benilan, A. Farooq, Quantitative mid-infrared spectra of allene and propyne from room to high temperatures, Journal of Molecular Spectroscopy 305 (2014) 10-16.

[119] M. Alrefae, A. Farooq, Absorption cross-section measurements of methane, ethane, ethylene and 
methanol at high temperatures, Journal of Molecular Spectroscopy 303 (2014) 8-14.

[120] Goldenstein, Christopher S., V. A. Miller, R. M. Spearrin, C. L. Strand, SpectraPlot.com: Spectroscopic Models for Atomic and Molecubart Gases, 2016.

[121] S. T. Sanders, J. A. Baldwin, T. P. Jenkins, D. S. Baer, R. K. Hanson, Diode-laser sensor for monitoring multiple combustion parameters in pulse detonation engines, Proceedings of the Combưstion Institute 28 (2000) 587-594.

[122] R. K. Hanson, D. F. Davidson, Recent advances in laser absorption and shock tube methods for studies of combustion chemistry, Progress in Energy and Combustion Science 44 (2014) 103-114.

[123] D. W. Mattison, J. B. Jeffries, R. K. Hanson, R. Steeper, S. De Zilwa, J. Dec, M. Sjoberg, W. Hwang, In-cylinder gas temperature and water concentration measurements in HCCI engines using a multiplexed-wavelength diode-laser system: Sensor development and initial demonstra2tion, Proceedings of the Combustion Institute 31 (2007) 791-798.

[124] M. Brown, G. Herring, K. Cabell, N. Hass, T. Barhorst, M. Gruber, Optical measurements at the combustor exit of the HIFiRE 2 group 323 test engine, in: 50th AIAA Aerospace Sciences Meeting including the New Horizons Forum and Aerospace Exposition, 2012, pp. AIAA 20120857.

[125] G. S. Jatana, M. Magee, D. Fain, S. V. Naiłks G. M. Shaver, R. P. Lucht, Simultaneous highspeed gas property measurements at the exhaust gas recirculation cooler exit and at the turbocharger inlet of a multicylinder diesel engine using diode-laser-absorption spectroscopy, Applied Optics 54 (2015) 1220-1231.

[126] K. M. Busa, B. Rice, J. C. McDaniel, C. P. Goyne, R. D. Rockwell, J. A. Fulton, J. R. Edwards, G. S. Diskin, Direct measurement of combustion efficiency of a dual-mode scramjet via TDLAT and SPIV, in: 53rd AIAA Aerospres Sciences Meeting, 2015, pp. AIAA 2015-0357. URL: http://arc.aiaa.org/doi/10.2514/6. 2015-0357. doi:10.2514/6.2015-0357.

[127] L. A. Kranendonk, R. Huber, J. G. Fujimoto, S. T. Sanders, Wavelength-agile $\mathrm{H}_{2} \mathrm{O}$ absorptiøæ spectrometer for thermometry of general combustion gases, Proceedings of the Combustion Institute 31 (2007) 783-790.
[128] N. G. Blume, S. Wagner, Broadband supercontinuum laser absorption spectrometer for multiparameter gas phase combustion diagnostics, Optics Letters 40 (2015) 3141-3144.

[129] J. A. Silver, D. J. Kane, Diode laser measurements of concentration and temperature in microgravity combustion, Measurement Science and Technology 10 (1999) 845-852.

[130] D. T. Cassidy, J. Reid, Atmospheric pressure monitoring of trace gases using tunable diode lasers, Applied Optics 21 (1982) 1185-1190.

[131] K. Sun, X. Chao, R. Sur, C. S. Goldenstein, J. B. Jeffries, R. K. Hanson, Analysis of calibrationfree wavelength- scanned wavelength modulation spectroscopy for practical gas sensing using tunable diode lasers, Measurement Science and Technology 24 (2013) 125203.

[132] C. S. Goldenstein, C. L. Strand, I. A. Schultz, K. Sun, J. B. Jeffries, R. K. Hanson, Fitting of calibration-free scanned-wavelength-modulation spectroscopy spectra for determination of gas properties and absorption lineshapes, Applied Optics 53 (2014) 356-367.

[133] Z. Qu, R. Ghorbani, D. Valiev, F. M. Schmidt, Calibration-free scanned wavelength modulation spectroscopy application to $\mathrm{H}_{2} \mathrm{O}$ and temperature sensing in flames, Optics Express 23 (2015) 16492-16499.

[134] P. Kluczynski, O. Axner, Theoretical description based on Fourier analysis of wavelengthmodulation spectrometry in terms of analytical and background signals, Applied Optics 38 (1999) 5803-5815.

[135] P. Kluczynski, Å. M. Lindberg, O. Axner, Characterization of background signals in wavelengthmodulation spectrometry in terms of a Fourier based theoretical formalism, Applied Optics 40 (2001) $770-782$.

[136] J. Gustafsson, O. Axner, 'Intelligent' triggering methodology for improved detectability of wavelength modulation diode laser absorption spectrometry applied to window-equipped graphite furnaces, Spectrochimica Acta Part B: Atomic Spectroscopy 58 (2003) 143-152.

[137] K. Sun, X. Chao, R. Sur, J. B. Jeffries, R. K. Hanson, Wavelength modulation diode laser absorption spectroscopy for high-pressure gas sensing, Applied Physics B 110 (2013) 497-508. 
[138] C. S. Goldenstein, C. A. Almodovar, J. B. Jeffries, R. K. Hanson, High-bandwidth scannedwavelength-modulation spectroscopy sensors for temperature and $\mathrm{H}_{2} \mathrm{O}$ in a rotating detonatioll engine, Measurement Science and Technology 25 (2014) 105104.

[139] J. A. Silver, Frequency-modulation spectroscopy for trace species detection: theory and comparison among experimental methods, Applied $\mathrm{O}_{\mathrm{p}-}^{4010}$ tics 31 (1992) 707-717.

[140] A. J. McGettrick, K. Duffin, W. Johnstone, G. Stewart, D. G. Moodie, Tunable diode laser spectroscopy with wavelength modulation: a phasor decomposition method for calibration-free measurements of gas concentration and pressure, Journal of Lightwave Technology 26 (2008) 432440.

[141] J. R. P. Bain, W. Johnstone, K. Ruxton, G. Stewart, M. Lengden, K. Duffin, Recovery of absolute gas absorption line shapes using tunable diode laser spectroscopy with wavelength modulation-Part 2: experimental investigation, Journal of Lightwave Technology 29 (2011) 987996.

[142] S. J. Harris, Intracavity laser spectroscopy: an old field with new prospects for combustion diagnostics, Applied Optics 23 (1984) 1311-1318.

[143] A. Goldman, I. Rahinov, S. Cheskis, B. Lohden. S. Wexler, K. Sengstock, V. M. Baev, Fiber laser intracavity absorption spectroscopy of ammonia and hydrogen cyanide in low pressure hydrocarbon flames, Chemical Physics Letters 423 (2006) 147-151.

[144] B. Lohden, S. Kuznetsova, K. Sengstock, V. M. Baev, A. Goldman, S. Cheskis, B. Palsdottir, Fiber laser intracavity absorption spectroscopy for in situ multicomponent gas analysis in the atmosphere and combustion environments, $\mathrm{A}_{4440}$ plied Physics B 102 (2011) 331-344.

[145] A. O'Keefe, J. J. Scherer, J. B. Paul, cw Integrated cavity output spectroscopy, Chemical Physics Letters 307 (1999) 343-349.

[146] J. B. Paul, L. Lapson, J. G. Anderson, Ultrasensitive absorption spectroscopy with a high-finesse optical cavity and off-axis alignment, Applied Optics 40 (2001) 4904-4910.

[147] J. B. Paul, J. J. Scherer, A. O'Keefe, L. Lapson, J. G. Anderson, C. F. Gmachl, F. Capassos, Infrared cavity ringdown and integrated cavity output spectroscopy for trace species monitoring, Proceedings of SPIE 4577 (2002) 1-11.

[148] E. Moyer, D. Sayres, G. Engel, J. St. Clair, F. Keutsch, N. Allen, J. Kroll, J. G. Anderson, Design considerations in high-sensitivity off-axis integrated cavity output spectroscopy, Applied Physics B 92 (2008) 467-474.

[149] P. Malara, M. F. Witinski, F. Capasso, J. G. Anderson, P. De Natale, Sensitivity enhancement of off-axis ICOS using wavelength modulation, Applied Physics B 108 (2012) 353-359.

[150] K. Sun, S. Wang, R. Sur, X. Chao, J. B. Jeffries, R. K. Hanson, Time-resolved in situ detection of $\mathrm{CO}$ in a shock tube using cavity-enhanced absorption spectroscopy with a quantum-cascade laser near 4.6 um, Optics Express 22 (2014) 24559-24565.

[151] K. Sun, S. Wang, R. Sur, X. Chao, J. B. Jeffries, R. K. Hanson, Sensitive and rapid laser diagnostic for shock tube kinetics studies using cavity-enhanced absorption spectroscopy, Optics Express 22 (2014) 1-11.

[152] S. Cheskis, A. Goldman, Laser diagnostics of trace species in low-pressure flat flame, Progress in Energy and Combustion Science 35 (2009) $365-382$.

[153] X. Mercier, P. Desgroux, Cavity Ring-Down Spectroscopy for Combustion studies, in: G. Berden, R. Engeln (Eds.), Cavity RingDown Spectroscopy: Techniques and Applications, John Wiley and Sons, 2010, pp. 273-311.

[154] K. D. Rein, S. Roy, B. Sell, J. L. Hoke, A. W. Caswell, F. R. Schauer, J. R. Gord, Timeresolved in-situ absorption spectroscopy of a hydrogen-air rotating detonation engine using a fiber-coupled tunable laser system, in: AIAA SciTech, 54th Aerospace Sciences Meeting, 2016, pp. AIAA 2016-1199.

[155] C. S. Goldenstein, R. M. Spearrin, J. B. Jeffries, R. K. Hanson, Wavelength-modulation spectroscopy near $2.5 \mu \mathrm{m}$ for $\mathrm{H}_{2} \mathrm{O}$ and temperature in high-pressure and -temperature gases, Applied Physics B 116 (2014) 705-716.

[156] E. L. Petersen, A Shock Tube and Diagnostics for Chemistry Measurements at Elevated Pressures with Application to Methane Ignition, Ph.D. thesis, Stanford University, 1998.

[157] L. A. Kranendonk, S. T. Sanders, Optical design in beam steering environments with emphasis on 
laser transmission measurements, Applied Optics 44 (2005) 6762-6772.

[158] J. M. Whitney, K. Takami, S. T. Sanders,

Y. Okura, Design of system for rugged, low-noise fiber-optic access to high-temperature, higkp pressure environments, IEEE Sensors Journal 11 (2011) 3295-3302.

[159] C. L. Strand, Scanned Wavelength-Modulation Absorption Spectroscopy with Application to Hypersonic Impulse Flow Facilities, Ph.D. thet sis, Stanford University, 2014.

[160] X. An, A. W. Caswell, J. J. Lipor, S. T. Sanders, Determining the optimum wavelength pairs to use for molecular absorption thermometry based on the continuous-spectral lower-state energy, Journal of Quantitative Spectroscopy and Radiative Transfer 112 (2011) 2355-2362.

[161] X. An, A. W. Caswell, S. T. Sanders, Quantifying the temperature sensitivity of practical spectra using a new spectroscopic quantity: Frequencydependent lower-state energy, Journal of Quantitative Spectroscopy and Radiative Transfer 112 (2011) 779-785.

[162] C. S. Goldenstein, R. M. Spearrin, I. a. Schultžcs J. B. Jeffries, R. K. Hanson, Wavelengthmodulation spectroscopy near $1.4 \mu \mathrm{m}$ for measurements of $\mathrm{H}_{2} \mathrm{O}$ and temperature in highpressure and -temperature gases, Measurement Science and Technology 25 (2014) 055101.

[163] M. S. Brittelle, J. M. Simms, S. T. Sanders, J. R. Gord, S. Roy, Fixed-wavelength $\mathrm{H}_{2} \mathrm{O}$ absorption spectroscopy system enhanced by an on-board external-cavity diode laser, Measurement Science and Technology 27 (2016) 035501.

[164] A. W. Caswell, S. Roy, X. An, S. T. Sanders, F. R. Schauer, J. R. Gord, Measurements of multiple gas parameters in a pulsed-detonation combustor using time-mode-locked lasers, Applied Optics 52 (2013) 2893-2904.

[165] O. Witzel, A. Klein, C. Meffert, S. Wagner, S. Kaiser, C. Schulz, V. Ebert, VCSEL-based, high-speed, in situ TDLAS for in-cylinder water vapor measurements in IC engines, Optics Express 21 (2013) 8057-8067.

[166] G. B. Rieker, H. Li, X. Liu, J. B. Jeffries, R. K. Hanson, M. G. Allen, S. D. Wehe, P. Mulhall, H. Kindle, A diode laser sensor for rapid, sensitive measurements of gas temperature and water vapour concentration at high temperatures and pressures, Measurement Science and Technology 18 (2007) 1195-1204.

[167] A. Farooq, J. B. Jeffries, R. K. Hanson, Measurements of $\mathrm{CO}_{2}$ concentration and temperature at high pressures using $1 f$-normalized wavelength modulation spectroscopy with second harmonic detection near $2.7 \mu \mathrm{m}$, Applied Optics 48 (2009) 6740-6753.

[168] S. H. Pyun, J. M. Porter, J. B. Jeffries, R. K. Hanson, J. C. Montoya, M. G. Allen, K. R. Sholes, Two-color-absorption sensor for timeresolved measurements of gasoline concentration and temperature, Applied Optics 48 (2009) 6492-6500.

[169] P. Kluczynski, J. Gustafsson, Å. M. Lindberg, O. Axner, Wavelength modulation absorption spectrometry an extensive scrutiny of the generation of signals, Spectrochimica Acta Part B: Atomic Spectroscopy 56 (2001) 1277-1354.

[170] J. A. Silver, A. C. Slanton, Optical interference fringe reduction in laser absorption experiments, Applied Optics 27 (1988) 1914-1916.

[171] C. S. Goldenstein, I. A. Schultz, J. B. Jeffries, R. K. Hanson, Two-color absorption spectroscopy strategy for measuring the column density and path average temperature of the absorbing species in nonuniform gases, Applied Optics 52 (2013) 7950-7962.

[172] M. Schoenung, R. K. Hanson, CO and temperature measurements in a flat flame by laser absorption spectroscopy and probe techniques, Combustion Science and Technology 24 (1980) 227237.

[173] X. Ouyang, P. L. Varghese, Line-of-sight absorption measurements of high temperature gases with thermal and concentration boundary layers, Applied Optics 28 (1989) 3979-3984.

[174] J. M. Seitzman, B. T. Scully, Broadband infrared absorption sensor for high-pressure combustor control, Journal of Propulsion and Power 16 (2000) 994-1001.

[175] J. Wang, M. Maiorov, J. B. Jeffries, D. Z. Garbuzov, J. C. Connolly, R. K. Hanson, A potential remote sensor of $\mathrm{CO}$ in vehicle exhausts using $2.3 \mu \mathrm{m}$ diode lasers, Measurement Science and Technology 11 (2000) 1576-1584.

[176] S. T. Sanders, J. Wang, J. B. Jeffries, R. K. Hanson, Diode-laser absorption sensor for line-ofsight temperature distributions, Applied Optics 40 (2001) 4404-4415. 
[177] T. I. Palaghita, J. M. Seitzman, Control of Tenbeo perature Nonuniformity Based on Line-of-Sight Absorption, in: 40th AIAA/ASME/SAE/ASEE Joint Propulsion Conference, Ft. Lauderdale, FL, 2004, pp. AIAA 2004-4163.

[178 J. T. C. Tin, Gruber, C. D. Carter, T. Mathur, R. K. Hanson, Near-infrared diode laser absorption diagnostic for temperature and water vapor in a scramjet combustor., Applied Optics 44 (2005) 67016711.

[179] X. Liu, J. B. Jeffries, R. K. Hanson, Measurement of non-uniform temperature distributions using line-of-sight absorption spectroscopy, AIAA Journal 45 (2007) 411-419.

[185] L. Ma, W. Cai, A. W. Caswell, T. Kraetschmer, S. T. Sanders, S. Roy, J. R. Gord, Tomographic imaging of temperature and chemical species based on hyperspectral absorption spectroscopy, Optics Express 17 (2009) 8602-86134250
[186] X. An, T. Kraetschmer, K. Takami, S. T. Sanders, L. Ma, W. Cai, X. Li, S. Roy, J. R. Gord, Validation of temperature imaging by $\mathrm{H}_{2} \mathrm{O}$ absorption spectroscopy using hyperspectral tomography in controlled experiments., Applied Optics 50 (2011) A29-37.

[187] K. Busa, E. Bryner, J. McDaniel, C. Goyne, C. Smith, G. Diskin, Demonstration of capability of water flux measurement in a scramjet combustor using tunable diode laser absorption tomography and stereoscopic PIV, 49th AIAA Aerospace Sciences Meeting including the New Horizons Forum and Aerospace Exposition (2011) AIAA 2011-1294.

[188] K. Busa, E. Ellison, B. McGovern, J. McDaniel, G. Diskin, M. DePiro, D. Capriotti, R. Gaffney, Measurements on NASA Langley durable combustor rig by TDLAT: preliminary results, 51st AIAA Aerospace Sciences Meeting including the New Horizons Forum and Aerospace Exposition (2013) AIAA 2013-0696.

[189] L. Ma, X. Li, S. T. Sanders, A. W. Caswell, S. Roy, D. H. Plemmons, J. R. Gord, 50-kHz-rate 2D imaging of temperature and $\mathrm{H}_{2} \mathrm{O}$ concentration at the exhaust plane of a J85 engine using hyperspectral tomography., Optics Express 21 (2013) 1152-1162.

[190] K. M. Busa, J. C. McDaniel, M. S. Brown, G. S. Diskin, Implementation of maximum-likelihood expectation-maximization algorithm for tomographic reconstruction of TDLAT measurements, AIAA SciTech, 52nd Aerospace Sciences Meeting (2014) AIAA 2014-0985.

[191] W. Cai, C. F. Kaminski, Multiplexed absorption tomography with calibration-free wavelength modulation spectroscopy, Applied Physics Letters 104 (2014) 154106.

[192] X. An, M. S. Brittelle, P. T. Lauzier, J. R. Gord, S. Roy, G.-H. Chen, S. T. Sanders, Demonstration of temperature imaging by $\mathrm{H}_{2} \mathrm{O}$ absorption spectroscopy using compressed sensing tomography, Applied Optics 54 (2015) 9190.

[193] C. Liu, L. Xu, J. Chen, Z. Cao, Y. Lin, Development of a fan-beam TDLAS-based tomographic sensor for rapid imaging of temperature and gas concentration, Optics Express 23 (2015) 48274842 .

[194] H. McCann, P. Wright, K. Daun, Chemical Species Tomography, in: M. Wang (Ed.), Industrial Tomography: Systems and Applications, Elsevier, 2015. 
[195] K. J. Daun, S. J. Grauer, P. J. Hadwin, Chenвbo ical species tomography of turbulent flows: Discrete ill-posed and rank deficient problems and the use of prior information, Journal of Quantitative Spectroscopy and Radiative Transfer 172 (2016) 58-74.

[196] R. Gordon, R. Bender, G. T. Herman, Algebraic reconstruction techniques (ART) for threedimensional electron microscopy and x-ray photography, Journal of Theoretical Biology 29 (1970) 471-481.

[197] A. C. Kak, M. Slaney, Principles of Computerized Tomographic Imaging, IEEE Press, 1988.

[198] A. H. Andersen, A. C. Kak, Simultaneous algebraic reconstruction technique (SART): a superior implementation of the ART algorithm, Ultrasonic Imaging 6 (1984) 81-94.

[199] A. J. Rockmore, A. Macovski, A maximum likelihood approach to emission image reconstruction from projections, IEEE Transactions on Nuclear Science 23 (1976) 1428-1432.

[200] E. Y. Sidky, X. Pan, Image reconstruction in circular cone-beam computed tomography by constrained, total-variation minimization, Physics in Medicine and Biology 53 (2008) 4777-4807.

[201] J. Tang, B. E. Nett, G.-H. Chen, Performance comparison between total variation (TV)-basêđ compressed sensing and statistical iterative reconstruction algorithms., Physics in Medicine and Biology 54 (2009) 5781-5804.

[202] P. C. Hansen, Rank-Deficient and Discrete IllPosed Problems: Numerical Aspects of Linear Inversion, 1998.

[203] W. Cai, D. J. Ewing, L. Ma, Application of simulated annealing for multispectral tomography, Computer Physics Communications 179 (2008) 250-255.

[204] L. Ma, X. Li, W. Cai, S. Roy, J. R. Gord, S. T35. Sanders, Selection of multiple optimal absorption transitions for nonuniform temperature sensing, Applied Spectroscopy 64 (2010) 1274-1282.

[205] X. Zhou, J. B. Jeffries, R. K. Hanson, Development of a fast temperature sensor for combustiơł gases using a single tunable diode laser, Applied Physics B 81 (2005) 711-722.

[206] A. Lytkine, W. Jäger, J. Tulip, Gas phase chemical analysis using long-wavelength vertical-cavity surface-emitting lasers, Optical Engineering 4 (2006) 044301.
[207] J. Chen, A. Hangauer, R. Strzoda, M. C. Amann, VCSEL-based calibration-free carbon monoxide sensor at $2.3 \mu \mathrm{m}$ with in-line reference cell, Applied Physics B 102 (2011) 381-389.

[208] R. Huber, M. Wojtkowski, K. Taira, J. Fujimoto, K. Hsu, Amplified, frequency swept lasers for frequency domain reflectometry and OCT imaging: design and scaling principles, Optics Express 13 (2005) 3513-3528.

[209] R. A. Huber, M. Wojtkowski, J. G. Fujimoto, Fourier Domain Mode Locking (FDML): A new laser operating regime and applications for optical coherence tomography., Optics Express 14 (2006) 3225-3237.

[210] A. W. Caswell, Water Vapor Absorption Thermometry for Practical Combustion Applications, Ph.D. thesis, University of Wisconsin, 2009.

[211] P. V. Kelkar, F. Coppinger, A. S. Bhushan, B. Jalali, Time domain optical sensing, in: IEEE Lasers and Electro-Optics Society 1999 12th Annual Meeting, volume 1, 1999.

[212] S. T. Sanders, Wavelength-agile fiber laser using group-velocity dispersion of pulsed supercontinua and application to broadband absorption spectroscopy, Applied Physics B 75 (2002) 799-802.

[213] J. W. Walewski, S. T. Sanders, High-resolution wavelength-agile laser source based on pulsed super-continua, Applied Physics B 79 (2004) $415-418$.

[214] C. Hagen, S. T. Sanders, Application of a novel white laser sensor to an HCCI engine, Society of Automotive Engines (2006).

[215] J. Hult, R. S. Watt, C. F. Kaminski, High bandwidth absorption spectroscopy with a dispersed supercontinuum source., Optics Express 15 (2007) 11385-11395.

[216] C. F. Kaminski, R. S. Watt, A. D. Elder, J. H. Frank, J. Hult, Supercontinuum radiation for applications in chemical sensing and microscopy, Applied Physics B 92 (2008) 367-378.

[217] T. Werblinski, F. Mittmann, M. Altenhoff, T. Seeger, L. Zigan, S. Will, Temperature and water mole fraction measurements by timedomain-based supercontinuum absorption spectroscopy in a flame, Applied Physics B: Lasers and Optics 118 (2014) 153-158. 
[218] N. G. Blume, V. Ebert, A. Dreizler, S. Wagner, Broadband fitting approach for the application of supercontinuum broadband laser absorption spectroscopy to combustion environments, Measurement Science and Technology 27 (201460) 015501.

[219] R. M. Spearrin, C. S. Goldenstein, I. A. Schultz, J. B. Jeffries, R. K. Hanson, Simultaneous sensing of temperature, $\mathrm{CO}$, and $\mathrm{CO}_{2}$ in a scramjet combustor using quantum cascade laser absorpeps tion spectroscopy, Applied Physics B 117 (2014) 689-698.

[220] C. S. Goldenstein, R. M. Spearrin, J. B. Jeffries, R. K. Hanson, Infrared laser absorption sensors for multiple performance parameters in a detonation combustor, Proceedings of the Combustion Institute 35 (2015) 3739-3747.

[221] J. Faist, F. Capasso, D. L. Sivco, C. Sirtori, A. L. Hutchinson, A. Y. Cho, Quantum cascade laser, Science 264 (1994) 553-556.

[222] J. Faist, C. Gmachl, F. Capasso, C. Sirtori, D. L. Sivco, J. N. Baillargeon, A. Y. Cho, Distributed feedback quantum cascade lasers, Ap-

[223] R. F. Curl, F. Capasso, C. Gmackrł, A. A. Kosterev, B. McManus, R. Lewicki, M. Pusharsky, G. Wysocki, F. K. Tittel, Quantum cascade lasers in chemical physics, Chemical

[224] B. S. Williams, Terahertz quantum-cascades lasers, Nature Photonics 1 (2007) 517-525.

[225] I. Vurgaftman, R. Weih, M. Kamp, J. R. Meyer, C. L. Canedy, C. S. Kim, M. Kim, W. W. Bewley, C. D. Merritt, J. Abell, S. Höfling, Interband cascade lasers, Journal of Physics D: Applieds Physics 48 (2015) 123001.

[226] R. Q. Yang, Infrared laser based on intersubband transitions in quantum wells, Superlattices and Microstructures 17 (1995) 77-83.

[227] M. Kim, C. L. Canedy, W. W. Bewley, C. Kim, J. R. Lindle, J. Abell, I. Vurgaftman, J. R. Meyer, Interband cascade laser emitting at $\lambda=3.75 \mu \mathrm{m}$ in continuous wave above room temperature, Applied Physics Letters 92 (2008) 191110.

4440

[228] I. Vurgaftman, W. W. Bewley, C. L. Canedy, C. D. Merritt, J. Abell, J. R. Meyer, Interband Cascade Lasers With Low Threshold Powers and
[229] A. Bauer, Shortened injector interband cascade lasers for 3.3- to 3.6- $\mu \mathrm{m}$ emission, Optical Engineering 49 (2010) 111117.

[230] M. Saad, Indium fluoride glass fibers, in: R. I. Epstein, M. Sheik-Bahae (Eds.), Proc. SPIE 8275, Laser Refrigeration of Solids V,, International Society for Optics and Photonics, 2012, p. $82750 \mathrm{D}$.

[231] J. M. Kriesel, N. Gat, B. E. Bernacki, R. L. Erikson, B. D. Cannon, T. L. Myers, C. M. Bledt, J. A. Harrington, Hollow core fiber optics for mid-wave and long-wave infrared spectroscopy, in: SPIE Defense, Security, and Sensing, International Society for Optics and Photonics, 2011, p. $80180 \mathrm{~V}$.

[232] T. Kanamori, Y. Terunuma, S. Takahashi, T. Miyashita, Chalcogenide glass fibers for mid-infrared transmission, Journal of Lightwave Technology 2 (1984) 607-613.

[233] R. Mitchell Spearrin, Mid-infrared laser absorption spectroscopy for carbon oxides in harsh environments, Ph.D. thesis, Stanford University, 2014.

[234] M. E. Thomas, R. I. Joseph, W. J. Tropf, Infrared transmission properties of sapphire, spinel, yttria, and ALON as a function of temperature and frequency, Applied Optics 27 (1988) 239245 .

[235] T. R. Harris, Optical Properties of Si, Ge, GaAs, GaSb, InAs, and InP at elevated temperatures, Ph.D. thesis, Air Force Institute of Technology, 2010.

[236] R. S. M. Chrystie, E. F. Nasir, A. Farooq, Towards simultaneous calibration-free and ultrafast sensing of temperature and species in the intrapulse mode, Proceedings of the Combustion Institute 35 (2014) 3757-3764.

[237] G. B. Rieker, F. R. Giorgetta, W. C. Swann, J. Kofler, A. M. Zolot, L. C. Sinclair, E. Baumann, C. Cromer, G. Petron, C. Sweeney, P. P. Tans, I. Coddington, N. R. Newbury, Frequencycomb-based remote sensing of greenhouse gases over kilometer air paths, Optica 1 (2014) 290298.

[238] P. Schroeder, R. Wright, S. Coburn, B. Sodergren, K. Cossel, S. Droste, G. Truong, E. Baumann, F. Giorgetta, I. Coddington, N. Newbury, G. Rieker, Dual frequency comb laser absorption spectroscopy in a $16 \mathrm{MW}$ gas turbine exhaust, 
Proceedings of the Combustion Institute (201460) In Press.

[239] S. D. Wehe, D. S. Baert, R. K. Hanson, Tunable diode-laser absorption measurements of temperature, velocity, and $\mathrm{H}_{2} \mathrm{O}$ in hypervelocity flows, in: 33rd Joint Propulsion Conference and Exhibisto Joint Propulsion Conferences, 1997, pp. AIAA 97-3267.

[240] S. D. Wehe, D. S. Baer, R. K. Hanson, Measurements of gas temperature and velocity in hypervelocity flows using diode-laser sensors, in: 20th AIAA Advanced Measurement and Ground Testing Technology Conference, 1998, pp. AIAA 98-2699. doi:AIAA-98-2699.

[242] K. D. Rein, S. T. Sanders, Fourier-transform absorption spectroscopy in reciprocating engines, Applied Optics 49 (2010) 4728-4734.

[243] I. A. Schultz, C. S. Goldenstein, C. L. Strand, J. B. Jeffries, R. K. Hanson, C. P. Goyne, Hypersonic scramjet testing via diode laser absorp2tion in a reflected shock tunnel, Propulsion and Power 30 (2014) 1586-1594.

[244] C. L. Strand, R. K. Hanson, Quantification of supersonic impulse flow conditions via highbandwidth wavelength modulation absorptiơsi spectroscopy, AIAA Journal 53 (2015) 29782987.

[245] Z. Wang, S. T. Sanders, Toward single ended absorption spectroscopy probes based on backscattering from rough surfaces: $\mathrm{H}_{2} \mathrm{O}$ vapor measufęo ments near $1350 \mathrm{~nm}$, Applied Physics B 121 (2015) 187-192.

[246] C. S. Goldenstein, R. M. Spearrin, R. K. Hanson, Fiber-coupled diode-laser sensors for calibrationfree stand-off measurements of gas temperaturas, pressure, and composition, Applied Optics 55 (2016) 479-484.

[247] O. Witzel, A. Klein, C. Meffert, C. Schulz,
[248] M. J. Hall, M. Koenig, A fiber-optic probe to measure precombustion in-cylinder fuel-air ratio fluctuations in production engines, Symposium (International) on Combustion 26 (1996) 26132618.

[249] E. Tomita, N. Kawahara, M. Shigenaga, A. Nishiyama, R. W. Dibble, In situ measurement of hydrocarbon fuel concentration near a spark plug in an engine cylinder using the 3.392 $\mathrm{m}$ infrared laser absorption method: discussion of applicability with a homogeneous methane-air mixture, Measurement Science and Technology 14 (2003) 1350-1356.

[250] M. Cundy, T. Schucht, O. Thiele, V. Sick, Highspeed laser-induced fluorescence and spark plug absorption sensor diagnostics for mixing and combustion studies in engines, Applied Optics 48 (2008) B94.

[251] H. Wang, A high-temperature chemical kinetic model of n-alkane (up to n-dodecane), cyclohexane, and methyl-, ethyl-, n-propyl and nbutyl-cyclohexane oxidation at high temperatures, JetSurF version 2.0., 2010. URL: http: //melchior.usc.edu/JetSurF.

[252] W. Ren, A. Farooq, D. F. Davidson, R. K. Hanson, CO concentration and temperature sensor for combustion gases using quantum-cascade laser absorption near $4.7 \mu \mathrm{m}$, Applied Physics B 107 (2012) 849-860.

[253] A. Farooq, J. B. Jeffries, R. K. Hanson, In situ combustion measurements of $\mathrm{H}_{2} \mathrm{O}$ and temperature near $2.5 \mu \mathrm{m}$ using tunable diode laser absorption, Measurement Science and Technology 19 (2008) 075604.

[254] A. Farooq, J. B. Jeffries, R. K. Hanson, Sensitive detection of temperature behind reflected shock waves using wavelength modulation spectroscopy of $\mathrm{CO}_{2}$ near $2.7 \mu \mathrm{m}$, Applied Physics B 96 (2009) $161-173$.

[255] W. Ren, J. B. Jeffries, R. K. Hanson, Temperature sensing in shock-heated evaporating aerosol using wavelength-modulation absorption spectroscopy of $\mathrm{CO}_{2}$ near $2.7 \mu \mathrm{m}$, Measurement Science and Technology 21 (2010) 105603.

[256] M. F. Campbell, T. Parise, A. M. Tulgestke, R. M. Spearrin, D. F. Davidson, R. K. Hanson, Strategies for obtaining long constant-pressure test times in shock tubes, Shock Waves 25 (2015) $651-665$. 
[257] M. E. MacDonald, W. Ren, Y. Zhu, D. F. Davidson, R. K. Hanson, Fuel and ethylene measurements during n-dodecane, methylcyclohexos ane, and iso-cetane pyrolysis in shock tubes, Fuel 103 (2013) 1060-1068.

[258] Y. Zhu, D. Davidson, R. Hanson, Pyrolysis and oxidation of decalin at elevated pressures: A shock-tube study, Combustion and Flame 1600 (2014) 371-383.

[259] W. Ren, D. F. Davidson, R. K. Hanson, IR laser absorption diagnostic for $\mathrm{C}_{2} \mathrm{H}_{4}$ in shock tube kinetics studies, International Journal of Chemical Kinetics 44 (2012) 423-432.

4605

[260] G. L. Pilla, D. F. Davidson, R. K. Hanson, Shock tube/laser absorption measurements of ethylene time-histories during ethylene and n-heptane pyrolysis, Proceedings of the Combustion Institute 33 (2011) 333-340.

[261] S. H. Pyun, J. Cho, D. F. Davidson, R. K. Hanson, Interference-free mid-IR laser absorption detection of methane, Measurement Science and

[265] U. KC, E. F. Nasir, A. Farooq, A mid-infrareed absorption diagnostic for acetylene detection, Applied Physics B 120 (2015) 223-232.

[266] A. B. S. Alquaity, E.-t. Es-sebbar, A. Farooq, Sensitive and ultra-fast species detection using pulsed cavity ringdown spectroscopy, Optics Eq press 23 (2015) 7217.

[267] P. Fjodorow, M. Fikri, C. Schulz, O. Hellmig, V. M. Baev, Time-resolved detection of tempera-
[268] S. Li, A. Farooq, R. K. Hanson, $\mathrm{H}_{2} \mathrm{O}$ temperature sensor for low-pressure flames using tunable diode laser absorption near $2.9 \mu \mathrm{m}$, Measurement Science and Technology 22 (2011) 125301.

[269] P. Nau, J. Koppmann, A. Lackner, K. KohseHöinghaus, A. Brockhinke, Quantum cascade laser-based MIR spectrometer for the determination of $\mathrm{CO}$ and $\mathrm{CO}_{2}$ concentrations and temperature in flames, Applied Physics B 118 (2015) $361-368$.

[270] R. Sur, K. Sun, J. B. Jeffries, J. G. Socha, R. K. Hanson, Scanned-wavelength-modulationspectroscopy sensor for $\mathrm{CO}, \mathrm{CO}_{2}, \mathrm{CH}_{4}$ and $\mathrm{H}_{2} \mathrm{O}$ in a high-pressure engineering-scale transportreactor coal gasifier, Fuel 150 (2015) 102-111.

[271] V. Ebert, J. Fitzer, I. Gerstenberg, K.-U. Pleban, H. Pitz, J. Wolfrum, M. Jochem, J. Martin, Simultaneous laser-based in-situ detection of oxygen and water in a waste incinerator for active combustion control purposes, Proceedings of the Combustion Institute 27 (1998) 1301-1308.

[272] V. Ebert, T. Fernholz, C. Giesemann, H. Pitz, H. Teichert, J. Wolfrum, H. Jaritz, Simultaneous diode-laser-based in situ detection of multiple species and temperature in a gas-fired power plant, Proceedings of the Combustion Institute 28 (2000) 423-429.

[273] H. Teichert, T. Fernholz, V. Ebert, Simultaneous in situ measurement of $\mathrm{CO}, \mathrm{H}_{2} \mathrm{O}$, and gas temperatures in a full-sized coal-fired power plant by near-infrared diode lasers, Applied Optics 42 (2003) 2043-2051.

[274] A. D. Sappey, Wavelength-multiplexed diode laser spectroscopy for closed loop combustion control and optimization, in: American-Japanese Flame Research Committees International Symposium, Waikaloa, 2007.

[275] X. Chao, J. B. Jeffries, R. K. Hanson, In situ absorption sensor for $\mathrm{NO}$ in combustion gases with a $5.2 \mu \mathrm{m}$ quantum-cascade laser, Proceedings of the Combustion Institute 33 (2011) 725-733.

[276] X. Chao, J. B. Jeffries, R. K. Hanson, Realtime, in situ, continuous monitoring of $\mathrm{CO}$ in a pulverized-coal-fired power plant with a $2.3 \mu \mathrm{m}$ laser absorption sensor, Applied Physics B 110 (2013) 359-365.

[277] T. P. Jenkins, J. Bergmans, Diode laser temperature measurements, in: J. Charles E. Baukal (Ed.), Industrial Combustion Testing, 
CRC Press Technology and Engineering, 2010, pp. 311-336.

[278] K. Sun, R. Sur, X. Chao, J. B. Jeffries, R. K. Hanson, R. J. Pummill, K. J. Whitty, TDL a absorption sensors for gas temperature and concentrations in a high-pressure entrained-flow coal gasifier, Proceedings of the Combustion Institute 34 (2013) 3593-3601.

[279] T. R. Meyer, S. Roy, T. N. Anderson, R. ${ }_{400}$ Lucht, R. Barron-Jimenez, J. R. Gord, 10 $\mathrm{kHz}$ detection of $\mathrm{CO}_{2}$ at $4.5 \mu \mathrm{m}$ by using tunable diode-laser-based difference-frequency generation, Optics Letters 30 (2005) 3087-3089.

[280] X. Liu, J. B. Jeffries, R. K. Hanson, K. M. Hinckos ley, M. A. Woodmansee, Development of a tunable diode laser sensor for measurements of gas turbine exhaust temperature, Applied Physics B 82 (2006) 469-478.

[281] A. W. Caswell, T. Kraetschmer, K. Rein, S. 410 Sanders, S. Roy, D. T. Shouse, J. R. Gord, Application of time-division-multiplexed lasers for measurements of gas temperature and $\mathrm{CH}_{4}$ and $\mathrm{H}_{2} \mathrm{O}$ concentrations at $30 \mathrm{kHz}$ in a high-pressure combustor, Applied Optics 49 (2010) 4963-4972.

[282] H. Li, S. D. Wehe, K. R. McManus, Real-time equivalence ratio measurements in gas turbine combustors with a near-infrared diode laser sensor, Proceedings of the Combustion Institute 33 (2011) 717-724.

4720

[283] X. Zhou, J. B. Jeffries, R. K. Hanson, G. Li, E. Gutmark, Wavelength-scanned tunable diode laser temperature measurements in a model gas turbine combustor, AIAA Journal 45 (2007) 420 425 .

[284] H. Li, X. Zhou, J. B. Jeffries, R. K. Hanson, Active control of lean blowout in a swirl-stabilized combustor using a tunable diode laser, Proceedings of the Combustion Institute 31 (2007) 32153223 .

[285] H. Li, X. Zhou, J. B. Jeffries, R. K. Hanson, Sensing and Control of Combustion Instabilities in Swirl-Stabilized Combustors Using Diode-Laser Absorption, AIAA Journal 45 (2007) 390-398 4735

[286] N. Terzija, S. Karagiannopoulos, S. Begg, P. Wright, K. Ozanyan, H. McCann, Tomographic imaging of the liquid and vapour fuel distributions in a single-cylinder direct-injection gasoline engine, International Journal of Enginae Research 16 (2015) 565-579.
[287] P. Wright, N. Terzija, J. L. Davidson, S. GarciaCastillo, C. Garcia-Stewart, S. Pegrum, S. Colbourne, P. Turner, S. D. Crossley, T. Litt, S. Murray, K. B. Ozanyan, H. McCann, Highspeed chemical species tomography in a multicylinder automotive engine, Chemical Engineering Journal 158 (2010) 2-10.

[288] O. Witzel, A. Klein, S. Wagner, C. Meffert, C. Schulz, V. Ebert, High-speed tunable diode laser absorption spectroscopy for sampling-free in-cylinder water vapor concentration measurements in an optical IC engine, Applied Physics B 109 (2012) 521-532.

[289] G. S. Jatana, S. V. Naik, G. M. Shaver, R. P. Lucht, High-speed diode laser measurements of temperature and water vapor concentration in the intake manifold of a diesel engine, International Journal of Engine Research 15 (2014) 773-788.

[290] L. A. Kranendonk, A. W. Caswell, S. T. Sanders, Robust method for calculating temperature, pressure, and absorber mole fraction from broadband spectra, Applied Optics 46 (2007) 41174124.

[291] A. D. Griffiths, A. F. P. Houwing, Diode laser absorption spectroscopy of water vapor in a scramjet combustor, Applied Optics 44 (2005) 66536659 .

[292] G. B. Rieker, J. T. C. Liu, J. B. Jeffries, R. K. Hanson, T. Mathur, M. R. Gruber, C. D. Carter, Diode laser sensor for gas temperature and $\mathrm{H}_{2} \mathrm{O}$ concentration in a scramjet combustor using wavelength modulation spectroscopy, in: 41st AIAA/ASME/SAE/ASEE Joint Propulsion Conference \& Exhibit, 2005, pp. AIAA 20053710 .

[293] F. Li, X. Yu, H. Gu, Z. Li, Y. Zhao, L. Ma, L. Chen, X. Chang, Simultaneous measurements of multiple flow parameters for scramjet characterization using tunable diode-laser sensors, Applied Optics 50 (2011) 6697-6707.

[294] I. A. Schultz, C. S. Goldenstein, R. M. Spearrin, J. B. Jeffries, R. K. Hanson, R. D. Rockwell, C. P. Goyne, Multispecies midinfrared absorption measurements in a hydrocarbon-fueled scramjet combustor, Journal of Propulsion and Power 30 (2014) 1595-1604.

[295] J. M. Meyers, D. Fletcher, Diode laser absorption sensor design and qualification for $\mathrm{CO}_{2}$ hypersonic flows, Journal of Thermophysics and Heat Transfer 25 (2011) 193-200. 
[296] I. A. Schultz, C. S. Goldenstein, J. B. Jeffries, R. K. Hanson, R. D. Rockwell, C. P. Goyne, Spatially resolved water measurements in a scramjet combustor using diode laser absorption, Journal of Propulsion and Power 30 (2014) 1551-1558 4795

[297] I. A. Schultz, C. S. Goldenstein, J. B. Jeffries, R. K. Hanson, R. D. Rockwell, C. P. Goyne, Diode laser absorption sensor for combustion progress in a model scramjet, Journal of Propulsion and Power 30 (2014) 550-557.

4800

[298] K. R. Jackson, M. R. Gruber, S. Buccellato, Mach 68+ hydrocarbon-fueled scramjet flight experiment: the HIFiRE flight 2 project, Journal of Propulsion and Power 31 (2015) 36-53.

[299] C. L. Strand, R. K. Hanson, Thermometry and velocimetry in supersonic flows via scanned wavelength-modulation absorption spectroscopy, in: 47th AIAA/ASME/SAE/ASEE

[300] D. W. Mattison, C. M. Brophy, S. T. Sanders, L. Ma, K. M. Hinckley, J. B. Jeffries, R. K. Hanson, Pulse detonation engine characterization and control using tunable diode-laser sensors, Journal of Propulsion and Power 19 (2003) $568-572$.

[301] A. W. Caswell, S. Roy, X. An, S. T. Sanders, High-bandwidth $\mathrm{H}_{2} \mathrm{O}$ absorption sensor for measuring pressure, enthalpy, and mass flux in a pulsed-detonation combustor, in: 50th AIAA Aerospace Sciences Meeting Including the New Horizons Forum and Aerospace Exposition, 2012, pp. AIAA 2012-0471.

[302] C. S. Goldenstein, I. A. Schultz, R. M. Spearrin, J. B. Jeffries, R. K. Hanson, Diode laser measurements of temperature and $\mathrm{H}_{2} \mathrm{O}$ for monitoring pulse detonation combustor performance, in: 24th International Colloquium on the Dynamics of Explosions and Reactive Systems, Taipei, 2013, pp. 1-6.

[303] R. M. Spearrin, C. S. Goldenstein, J. B. Jeffries, R. K. Hanson, Mid-infrared laser absorption diagnostics for detonation studies, in: 29th International Symposium on Shock Waves, Madison, 2013.

[304] J. Cheng, M. E. V. Pedersen, K. Wang, C. Xu, L. Grüner-Nielsen, D. Jakobsen, Time-domain multimode dispersion measurement in a higherorder-mode fiber, Optics Letters 37 (2012) 347.
[305] D. A. Schwer, K. Kailasanath, Modeling exhaust effects in rotating detonation engines, in: 48th AIAA/ASME/SAE/ASEE Joint Propulsion Conference and Exhibit, 2012, pp. AIAA 2012-3943.

[306] C. J. Mcgahan, B. A. Tom, A. W. Caswell, J. R. Gord, F. R. Schauer, J. L. Hoke, Exhaust gas analysis of a rotating detonation engine using tunable diode laser absorption spectroscopy, in: AIAA SciTech, 52nd Aerospace Sciences Meeting, 2014, pp. AIAA 2014-0391.

[307] B. A. Rankin, D. R. Richardson, A. W. Caswell, A. Naples, Imaging of $\mathrm{OH}^{*}$ chemiluminescence in an optically accessible rotating detonation engine, 53rd AIAA Aerospace Sciences Meeting (2015) AIAA 2015-1604. 